\title{
A reaction-diffusion equation on a thin $L$-shaped domain
}

\author{
Jack K. Hale \\ School of Mathematics, Georgia Institute of Technology, Atlanta, \\ GA 30332, U.S.A.
}

\section{Geneviève Raugel}

Laboratoire d'Analyse Numérique, Bâtiment 425, Université Paris-Sud, 91405 Orsay cédex, France

(MS received 14 October 1993. Revised MS received 8 December 1993)

\begin{abstract}
We consider a dissipative reaction-diffusion equation on a thin L-shaped domain (with the thinness measured by a parameter $\varepsilon$ ); we determine the limit equation for $\varepsilon=0$ and prove the upper semicontinuity of the global attractors at $\varepsilon=0$. We also state a lower semicontinuity result. When the limit equation is one-dimensional, we prove convergence of any orbit to a singleton.
\end{abstract}

\section{Introduction}

In many applications, we encounter partial differential equations (PDE) defined on domains for which the size in some directions is much larger than the size in others. It is natural in such situations to attempt to determine a PDE on a lower-dimensional domain which will reflect all of the dynamics of the original problem. For very general domains which are thin in the normal direction over a lower dimensional bounded domain $\Omega$, Hale and Raugel $[12,13,15,16]$ have discussed this problem in detail for a dissipative parabolic equation and for a linearly damped hyperbolic equation. In particular, if the order of thinness is measured by $\varepsilon$, they constructed a limit problem on the lower-dimensional domain and proved upper semicontinuity of the attractors at $\varepsilon=0$. For gradient systems, it is also known that the attractors are lower semicontinuous at $\varepsilon=0$ provided that the equilibrium points are hyperbolic $[16,29]$. Raugel and Sell $[30,31]$ have considered similar problems (including global existence) for the Navier-Stokes equations on a three-dimensional bounded domain $\Omega \times(0, \varepsilon)$. Thin domain problems have also been considered by several other authors from different points of view; see, for instance $[4,22,25,26]$ and the references therein. For time-dependent problems, see also [28].

In this paper, we prove the upper semicontinuity of the attractors as well as other properties of a dissipative parabolic equation on thin L-shaped domains. A very special case of such a domain in $\mathbb{R}^{2}$ is the set $\left\{\left(x_{1}, x_{2}\right): 0<x_{1}<1,0<\right.$ $\left.x_{2}<\varepsilon\right\} \cup\left\{\left(x_{1}, x_{2}\right): 0<x_{1}<\varepsilon, 0<x_{2}<1\right\}$. The junction region of this domain is defined to be $(0, \varepsilon) \times(0, \varepsilon)$.

Ciarlet [4] and Le Dret [23-25] have considered such problems for linear and 
nonlinear PDE, including problems in mechanics concerning shells, plates and rods. The first problem that is encountered is to scale the domain in such a way as to obtain problems on domains which are independent of $\varepsilon$. For the above simple example, the idea of Ciarlet and Le Dret is to scale the different parts of the domain independently of each other, but counting the junction twice. The idea of scaling the different parts of a multi-structure independently of each other, but counting the junction twice, first appeared in the work of Ciarlet, Le Dret and Nzengwa $[5,6]$. They obtained two domains $Q^{1}=(0,1) \times(0,1), Q^{2}=(0,1) \times(0,1)$ with the junction being $J_{\varepsilon}^{1}=(0, \varepsilon) \times(0,1)$ in $Q^{1}$ and $J_{\varepsilon}^{2}=(0,1) \times(0, \varepsilon)$ in $Q^{2}$. For the resulting PDE on the product domain, a solution $u=\left(u_{1}, u_{2}\right)$ is required to satisfy a junction condition in order to yield correct information about the original problem. A natural limit problem as $\varepsilon \rightarrow 0$ in this case consists of two one-dimensional PDE with a matching condition at the origin. The above authors discussed convergence of solutions on finite time intervals as $\varepsilon \rightarrow 0$. Other approaches to such problems are considered in [3.7], for example. One could also apply the techniques of asymptotic developments (see [4]).

Here, we use a type of scaling procedure similar to that of Ciarlet and Le Dret, but for a more general L-shaped domain, we determine the appropriate limit equations and then extend the method used by Hale and Raugel [13] to prove the upper semicontinuity of the attractors at $\varepsilon=0$. To assist in the analysis, Hale and Raugel $[12,13]$ used a projection operator from the higher-dimensional space to the lower-dimensional space given by the mean value with respect to $x_{2}$. Also, for thin domains, the identity map is a natural embedding of functions on the lowerdimensional space into functions on the canonical domain in the higher-dimensional space. In the above coordinates, this embedding takes a function of $\varphi\left(x_{1}\right)$ into a function $\psi\left(x_{1}, x_{2}\right)=\varphi\left(x_{1}\right)$ for all $x_{2}$. Due to the junction conditions on L-shaped domains, these artifacts will not work and this makes the analysis more difficult. The detailed reason for this is described in the text.

An outline of the paper is as follows. In Section 2, we describe the variational problems $(\tilde{\mathrm{P}})_{\varepsilon}$ and $(\tilde{\mathrm{P}})_{0}$ corresponding, respectively, to the reaction-diffusion equations on the thin L-shaped domain and to the limit problem. There we also introduce the main notation and state the upper semicontinuity result of the attractors $\mathscr{A}_{\varepsilon}$ of the problems $(\tilde{\mathrm{P}})_{\varepsilon}$ at $\varepsilon=0$. In Section 3 , we introduce our important auxiliary mappings $M_{\varepsilon}$ and $I_{\varepsilon}$. Sections 4-7 are devoted to the proof of the upper semicontinuity result. In Section 8 , we compare the equilibrium points of the problems $(\tilde{\mathrm{P}})_{\varepsilon}$ and $(\tilde{\mathrm{P}})_{0}$, when the equilibrium points of $(\tilde{\mathbf{P}})_{0}$ are hyperbolic. Section 9 is devoted to the comparison of the eigenvalues of the linearised problems corresponding to $(\tilde{\mathrm{P}})_{\varepsilon}$ and $(\tilde{\mathrm{P}})_{0}$. We first give abstract results of comparison of eigenvalues, which can be used in other situations. For special thin domains, Bourquin and Ciarlet [2] have also made comparison of eigenvalues. We then describe two main applications of our comparison results. The first application is the convergence, to a single equilibrium point, for each orbit of the reaction-diffusion equation on our thin L-shaped domain. The second application is the lower semicontinuity of the attractors $\mathscr{A}_{\varepsilon}$ at $\varepsilon=0$. Finally, in Section 10, we describe some generalisations to more complicated two-dimensional thin L-shaped domains and to three-dimensional thin L-shaped domains. 


\section{Notation and upper semicontinuity results}

For $g_{i} \in C^{2}([0,1] ;(0, \infty)), i=1,2$, and $\varepsilon \in(0,1]$, we define a general L-shaped domain $Q_{\varepsilon}$ by the relations

$$
\begin{aligned}
& Q_{\varepsilon}=Q_{\varepsilon}^{1} \cup Q_{\varepsilon}^{2}, \\
& Q_{\varepsilon}^{1}=\left\{\left(\tilde{x}_{1}, \tilde{x}_{2}\right) \in \mathbb{R}^{2}: 0<\tilde{x}_{2}<\varepsilon g_{1}\left(\tilde{x}_{1}\right), 0<\tilde{x}_{1}<1\right\}, \\
& Q_{\varepsilon}^{2}=\left\{\left(\tilde{x}_{1}, \tilde{x}_{2}\right) \in \mathbb{R}^{2}: 0<\tilde{x}_{1}<\varepsilon g_{2}\left(\tilde{x}_{2}\right), 0<\tilde{x}_{2}<1\right\} .
\end{aligned}
$$

We denote the closure of a set $S$ by $\bar{S}$. The set $\bar{J}_{\varepsilon}=\overline{Q_{\varepsilon}^{1} \cap Q_{\varepsilon}^{2}}$ is called the junction set of $Q_{\varepsilon}$ and is the closure of the open set

$$
J_{\varepsilon}=\left\{\left(\tilde{x}_{1}, \tilde{x}_{2}\right): 0<\tilde{x}_{2}<\varepsilon g_{1}\left(\tilde{x}_{1}\right), 0<\tilde{x}_{1}<\varepsilon g_{2}\left(\tilde{x}_{2}\right), \tilde{x}_{1} \in(0,1), \tilde{x}_{2} \in(0,1)\right\} .
$$

We set

$$
\Gamma_{\varepsilon}=\Gamma_{\varepsilon}^{1} \cup \Gamma_{\varepsilon}^{2}, \quad \Gamma_{\varepsilon}^{1}=\partial Q_{\varepsilon}^{1} \cap\left\{\tilde{x}_{1}=1\right\}, \quad \Gamma_{\varepsilon}^{2}=\partial Q_{\varepsilon}^{2} \cap\left\{\tilde{x}_{2}=1\right\} .
$$

Suppose that $G \in W^{1, \infty}(\tilde{Q})$, where $\tilde{Q} \supset \cup_{0<\varepsilon \leqq 1} \bar{Q}_{\varepsilon}$. The conditions on $G$ can be weakened (see the detailed proofs in the next sections). Let $f \in C^{2}(\mathbb{R} ; \mathbb{R})$ be given and suppose that there are constants $c>0,0 \leqq \gamma<\infty$, such that

$$
\begin{gathered}
\left|f^{\prime \prime}(s)\right| \leqq c\left(1+|s|^{\gamma}\right) \quad \text { for } s \in R, \\
\limsup _{|s| \rightarrow+\infty} \frac{-f(s)}{s}<0 .
\end{gathered}
$$

In view of the results of the first part of Section 9, the condition (2.2) can obviously be replaced by the following one:

$$
\limsup _{|s| \rightarrow+\infty} \frac{-f(s)}{s}<\lambda<\lambda_{10},
$$

where $\lambda_{10}$ is the first (positive) eigenvalue of the operator $A_{0}$ defined by $(2.5)_{0}$. Then, of course, one has to choose $\varepsilon$ small enough to ensure that the first eigenvalue $\lambda_{1 \varepsilon}$ of $-\Delta$ on $Q_{\varepsilon}$ (with the boundary conditions $u=0$ in $\Gamma_{\varepsilon}, \partial u / \partial n_{\varepsilon}=0$ in $\partial Q_{\varepsilon} \backslash \Gamma_{\varepsilon}$ ) satisfies the inequality $\lambda<\lambda_{1 \varepsilon}$. For the sake of simplicity, we make the hypothesis (2.2).

We consider the parabolic boundary value problem

$$
\begin{array}{ll}
u_{t}-\Delta u=-f(u)-G & \text { in } Q_{\varepsilon}, \\
u=0 & \text { in } \Gamma_{\varepsilon}, \\
\frac{\partial u}{\partial n_{\varepsilon}}=0 & \text { in } \partial Q_{\varepsilon} \backslash \Gamma_{\varepsilon},
\end{array}
$$

where $n_{\varepsilon}$ is the unit outward normal to $Q_{\varepsilon}$. The initial data are chosen from the space

$$
H_{\Gamma_{\varepsilon}}^{1}\left(Q_{\varepsilon}\right)=\left\{u \in H^{1}\left(Q_{\varepsilon}\right): u=0 \text { in } \Gamma_{\varepsilon}\right\} .
$$

Let us now write the problem (P) in variational form. For this, as has been done by Le Dret [25], it is convenient to write the inner product in the space $L^{2}\left(Q_{\varepsilon}\right)$ in 
the form

$$
\begin{aligned}
(u, v)_{L^{2}\left(Q_{\varepsilon}\right)}= & \int_{Q_{\varepsilon}^{1} \backslash J_{\varepsilon}} u v d \tilde{x}_{1} d \tilde{x}_{2}+\frac{1}{2} \int_{J_{\varepsilon}} u v d \tilde{x}_{1} d \tilde{x}_{2} \\
& +\int_{Q_{\varepsilon}^{2} \backslash J_{\varepsilon}} u v d \tilde{x}_{1} d \tilde{x}_{2}+\frac{1}{2} \int_{J_{\varepsilon}} u v d \tilde{x}_{1} d \tilde{x}_{2} .
\end{aligned}
$$

With this notation, we can see that a function $u$ is a solution to (P) if and only if, for all $w \in H_{\Gamma_{\varepsilon}}^{1}\left(Q_{\varepsilon}\right)$, we have

$$
\left(u_{t}, w\right)_{L^{2}\left(Q_{\varepsilon}\right)}+(\nabla u, \nabla w)_{L^{2}\left(Q_{\varepsilon}\right)}=-(f(u)+G, w)_{L^{2}\left(Q_{\varepsilon}\right)} .
$$

To discuss the problem $(P)$ or, equivalently $(\tilde{\mathrm{P}})$, it is convenient to transform coordinates to a canonical product domain $Q \equiv Q^{1} \times Q^{2}, Q^{1}=Q^{2}=(0,1) \times(0,1)$. To accomplish this, we need some notation. Let

$$
\begin{aligned}
& \varphi_{\varepsilon}^{1}:\left(x_{1}, x_{2}\right) \in \bar{Q}^{1} \mapsto\left(x_{1}, \varepsilon g_{1}\left(x_{1}\right) x_{2}\right) \in \bar{Q}_{\varepsilon}^{1}, \\
& \varphi_{\varepsilon}^{2}:\left(x_{1}, x_{2}\right) \in \bar{Q}^{2} \mapsto\left(\varepsilon g_{2}\left(x_{2}\right) x_{1}, x_{2}\right) \in \bar{Q}_{\varepsilon}^{2},
\end{aligned}
$$

and define the map $\varphi_{\varepsilon}: \bar{Q}^{1} \cup \bar{Q}^{2} \rightarrow \bar{Q}_{\varepsilon}$ as $\varphi_{\varepsilon} \mid \bar{Q}^{j}=\varphi_{\varepsilon}^{j}, j=1,2$. Clearly the map $\varphi_{\varepsilon}^{-1}: \bar{Q}_{\varepsilon} \rightarrow \bar{Q}^{1} \cup \bar{Q}^{2}$ exists. We have

$$
\begin{aligned}
& \left(\varphi_{\varepsilon}^{1}\right)^{-1} \Gamma_{\varepsilon}^{1} \equiv \Gamma^{1}=\left\{\left(1, x_{2}\right), 0<x_{2}<1\right\}, \\
& \left(\varphi_{\varepsilon}^{2}\right)^{-1} \Gamma_{\varepsilon}^{2} \equiv \Gamma^{2}=\left\{\left(x_{1}, 1\right), 0<x_{1}<1\right\} .
\end{aligned}
$$

We now want to determine $\varphi_{\varepsilon}^{-1} J_{\varepsilon}$.

From the Implicit Function Theorem, there exists a constant $\varepsilon_{0}>0$ and a neighbourhood $W$ of $(0,0)$ in $\mathbb{R}^{2}$ such that, for $0<\varepsilon \leqq \varepsilon_{0}$, the equation $x_{1}-\varepsilon g_{2}\left(\varepsilon g_{1}\left(x_{1}\right) x_{2}\right)=0$ has a unique solution $x_{1}\left(\varepsilon, x_{2}\right)$ in $W$ for $0 \leqq x_{2} \leqq 1$ with $x_{1}\left(0, x_{2}\right)=0$ and the equation $x_{2}-\varepsilon g_{1}\left(\varepsilon g_{2}\left(x_{2}\right) x_{1}\right)=0$ has a unique solution $x_{2}\left(\varepsilon, x_{1}\right)$ in $W$ for $0 \leqq x_{1} \leqq 1$ with $x_{2}\left(0, x_{1}\right)=0$. Moreover, the functions $x_{1}\left(\varepsilon, x_{2}\right)$ and $x_{2}\left(\varepsilon, x_{1}\right)$ are of class $C^{2}$ from $\left[0, \varepsilon_{0}\right] \times[0,1]$ into $W$. Also, it is not difficult to show that there is a positive constant $C$ such that, for $0<\varepsilon \leqq \varepsilon_{0}$, we have

$$
\begin{aligned}
& 0<x_{1}\left(\varepsilon, x_{2}\right) \leqq C \varepsilon, \quad\left|\frac{\partial x_{1}\left(\varepsilon, x_{2}\right)}{\partial x_{2}}\right| \leqq C \varepsilon^{2}, \quad \lim _{\varepsilon \rightarrow 0} \frac{1}{\varepsilon^{2}} \frac{\partial x_{1}\left(\varepsilon, x_{2}\right)}{\partial x_{2}}=g_{2}^{\prime}(0) g_{1}(0), \\
& 0<x_{2}\left(\varepsilon, x_{1}\right) \leqq C \varepsilon, \quad\left|\frac{\partial x_{2}\left(\varepsilon, x_{1}\right)}{\partial x_{1}}\right| \leqq C \varepsilon^{2}, \\
& \lim _{\varepsilon \rightarrow 0} \frac{1}{\varepsilon^{2}} \frac{\partial x_{2}\left(\varepsilon, x_{1}\right)}{\partial x_{1}}=g_{1}^{\prime}(0) g_{2}(0) .
\end{aligned}
$$

Since $\min _{x_{i} \in[0,1]} g_{i}\left(x_{i}\right)>0$, for $i=1$, 2, we can also show that there exists a positive constant $C_{0}$ such that, for $0<\varepsilon \leqq \varepsilon_{0}$,

$$
\begin{array}{ll}
x_{1}\left(\varepsilon, x_{2}\right) \geqq C_{0} \varepsilon, & 0 \leqq x_{2} \leqq 1, \\
x_{2}\left(\varepsilon, x_{1}\right) \geqq C_{0} \varepsilon, & 0 \leqq x_{1} \leqq 1 .
\end{array}
$$

This allows us to determine $\varphi_{\varepsilon}^{-1} J_{\varepsilon}$ :

$$
\begin{aligned}
\varphi_{\varepsilon}^{-1} J_{\varepsilon} & =J_{\varepsilon}^{1} \cup J_{\varepsilon}^{2}, \\
\left(\varphi_{\varepsilon}^{1}\right)^{-1} J_{\varepsilon} & \equiv J_{\varepsilon}^{1}=\left\{\left(x_{1}, x_{2}\right) \in Q^{1}: 0<x_{1}<x_{1}\left(\varepsilon, x_{2}\right), 0<x_{2}<1\right\},
\end{aligned}
$$




$$
\left(\varphi_{\varepsilon}^{2}\right)^{-1} J_{\varepsilon} \equiv J_{\varepsilon}^{2}=\left\{\left(x_{1}, x_{2}\right) \in Q^{2}: 0<x_{2}<x_{2}\left(\varepsilon, x_{1}\right), 0<x_{1}<1\right\} .
$$

We now introduce some spaces which arise naturally from the transformation $\varphi_{\varepsilon}^{-1}: Q_{\varepsilon} \rightarrow Q^{1} \cup Q^{2}$. We let $H \equiv L^{2}(Q) \equiv L^{2}\left(Q^{1}\right) \times L^{2}\left(Q^{2}\right)$ and we introduce the space $H_{\varepsilon} \equiv H_{\varepsilon}\left(Q, J_{\varepsilon}\right) \simeq H$ with the norm $\|\cdot\|_{H_{\varepsilon}}$ induced by the inner product

$$
(\xi, \zeta)_{H_{\varepsilon}}=\sum_{j=1}^{2}\left(\int_{Q^{j} \backslash \backslash_{\varepsilon}^{j}} g_{j} \xi_{j} \zeta_{j} d x_{1} d x_{2}+\frac{1}{2} \int_{J_{\varepsilon}^{j}} g_{j} \xi_{j} \zeta_{j} d x_{1} d x_{2}\right),
$$

where $\xi=\left(\xi_{1}, \zeta_{2}\right), \zeta=\left(\zeta_{1}, \zeta_{2}\right)$. We let $H^{1}(Q)=H^{1}\left(Q^{1}\right) \times H^{1}\left(Q^{2}\right)$ and we denote by $H_{\varepsilon}^{1}(Q)$ the space $H^{1}(Q)$ equipped with the norm $\|\cdot\|_{H_{\varepsilon}^{1}(Q)}$ defined by

$$
\|u\|_{H_{\varepsilon}^{1}(Q)}=\left(\|u\|_{H^{1}(Q)}^{2}+\frac{1}{\varepsilon^{2}}\left\|\frac{\partial u_{1}}{\partial x_{2}}\right\|_{L^{2}\left(Q^{1}\right)}^{2}+\frac{1}{\varepsilon^{2}}\left\|\frac{\partial u_{2}}{\partial x_{1}}\right\|_{L^{2}\left(Q^{2}\right)}^{2}\right)^{\frac{1}{2}}
$$

for $\quad u=\left(u_{1}, u_{2}\right)$. Let $\quad H_{\Gamma^{j}}^{1}\left(Q^{j}\right)=\left\{u \in H^{1}\left(Q^{j}\right): u \mid \Gamma^{j}=0\right\}, \quad j=1,2$. We let $V=H_{\Gamma}^{1}\left(Q^{1}\right) \times H_{\Gamma^{2}}^{1}\left(Q^{2}\right)$ and

$$
\begin{aligned}
& \mathscr{H}_{\varepsilon} \equiv \mathscr{H}_{\varepsilon}(Q) \equiv\left\{\left(\xi_{1}, \xi_{2}\right) \in H_{\varepsilon}: \xi_{1} \circ\left(\varphi_{\varepsilon}^{1}\right)^{-1}\left|J_{\varepsilon}=\xi_{2}^{\circ}\left(\varphi_{\varepsilon}^{2}\right)^{-1}\right| J_{\varepsilon} \text { a.e. }\right\}, \\
& \mathscr{V}_{\varepsilon} \equiv \mathscr{V}_{\varepsilon}(Q) \equiv\left\{\left(\xi_{1}, \xi_{2}\right) \in V: \xi_{1}{ }^{\circ}\left(\varphi_{\varepsilon}^{1}\right)^{-1}\left|J_{\varepsilon}=\xi_{2} \circ\left(\varphi_{\varepsilon}^{2}\right)^{-1}\right| J_{\varepsilon} \text { a.e. }\right\} .
\end{aligned}
$$

We can equip $\mathscr{V}_{\varepsilon}$ with the norm of $\|\cdot\|_{H_{\varepsilon}^{1}(Q)}$, but later we equip $\mathscr{Y}_{\varepsilon}$ with a more adequate norm.

If we now make the change of variables $\varphi_{\varepsilon}$ and let $G_{1 \varepsilon}\left(x_{1}, x_{2}\right)=G\left(x_{1}, \varepsilon g_{1}\left(x_{1}\right) x_{2}\right)$, $G_{2 \varepsilon}\left(x_{1}, x_{2}\right)=G\left(\varepsilon g_{2}\left(x_{2}\right) x_{1}, x_{2}\right)$, then the variational problem ( $\left.\tilde{\mathrm{P}}\right)$ is equivalent to finding a function $u^{\varepsilon} \in \mathscr{V}_{\varepsilon}$ such that, for every $w \in \mathscr{V}_{\varepsilon}$, we have

$$
\left(u_{t}^{\varepsilon}, w\right)_{H_{\varepsilon}}+a_{\varepsilon}\left(u^{\varepsilon}, w\right)=-\left(\hat{f}\left(u^{\varepsilon}\right)+\hat{G}_{\varepsilon}, w\right)_{H_{\varepsilon}},
$$

where $\hat{f}(u), \hat{G}_{\varepsilon}$ belong to $\mathscr{H}_{\varepsilon}$ with

$$
\hat{f}(u)=\left(f\left(u_{1}\right), f\left(u_{2}\right)\right), \quad \hat{G}_{\varepsilon}=\left(G_{1 \varepsilon}, G_{2 \varepsilon}\right),
$$

and the bilinear form $a_{\varepsilon}(\xi, \zeta)$ is defined as follows. If

$$
\underline{\xi}=\left[\begin{array}{l}
\xi^{1} \\
\xi^{2}
\end{array}\right]=\left[\begin{array}{l}
\left(\xi_{1}^{1}, \xi_{2}^{1}\right) \\
\left(\xi_{1}^{2}, \xi_{2}^{2}\right)
\end{array}\right], \quad \underline{\zeta}=\left[\begin{array}{l}
\zeta^{1} \\
\zeta^{2}
\end{array}\right]=\left[\begin{array}{l}
\left(\zeta_{1}^{1}, \zeta_{2}^{1}\right) \\
\left(\zeta_{1}^{2}, \zeta_{2}^{2}\right)
\end{array}\right],
$$

are two vectors in $H_{\varepsilon} \times H_{\varepsilon}$, we set

$$
(\underline{\xi}, \underline{\zeta})_{H_{\varepsilon}}=\sum_{j=1}^{2}\left[\int_{Q^{1} \backslash J_{\varepsilon}^{1}} g_{1} \xi_{1}^{j} \zeta_{1}^{j}+\frac{1}{2} \int_{J_{\varepsilon}^{1}} g_{1} \xi_{1}^{j} \zeta_{1}^{j}+\int_{Q^{2} \backslash J_{\varepsilon}^{2}} g_{2} \xi_{2}^{j} \zeta_{2}^{j}+\frac{1}{2} \int_{I_{\varepsilon}^{2}} g_{2} \xi_{2}^{j} \zeta_{2}^{j}\right] .
$$

For $\xi=\left(\xi_{1}, \xi_{2}\right) \in \mathscr{V}_{\varepsilon}$, we introduce the 'gradient vector'

$$
\begin{aligned}
\mathscr{L}_{\varepsilon}^{\frac{1}{\varepsilon}} \xi & =\left(\mathscr{L}_{\frac{1}{1} \varepsilon}^{\frac{1}{2}} \xi_{1}, \mathscr{L}_{\frac{1}{2} \varepsilon} \xi_{2}\right) \\
& =\left[\begin{array}{l}
\left(\xi_{1 x_{1}}-\frac{g_{1 x_{1}}}{g_{1}} x_{2} \xi_{1 x_{2}}, \frac{1}{\varepsilon g_{2}} \xi_{2 x_{1}}\right) \\
\left(\frac{1}{\varepsilon g_{1}} \xi_{1 x_{2}}, \xi_{2 x_{2}}-\frac{g_{2 x_{2}}}{g_{2}} x_{1} \xi_{2 x_{1}}\right)
\end{array}\right]
\end{aligned}
$$


and, for $\xi \in \mathscr{V}_{\varepsilon}, \zeta \in \mathscr{V}_{\varepsilon}$, we set

$$
a_{\varepsilon}(\xi, \zeta)=\left(\mathscr{L}_{\varepsilon}^{\frac{1}{2}} \xi, \mathscr{L}_{\varepsilon}^{\frac{1}{2}} \zeta\right)_{H_{\varepsilon}}
$$

We equip $\mathscr{V}_{\varepsilon}$ with the norm $\|\cdot\|_{\mathscr{V}_{\varepsilon}}$ defined by $\|u\|_{\mathscr{V}_{\varepsilon}}=\left(a_{\varepsilon}(u, u)\right)^{\frac{1}{2}}$. Actually, we can define $a_{\varepsilon}\left(u_{1}, u_{2}\right)$ if $u_{1}, u_{2}$ are only elements of $V$. For this reason, we denote by $V_{\varepsilon}=$ $V_{\varepsilon}(Q)$ the space $V$ equipped with the norm $\|u\|_{V_{\varepsilon}}=\left(a_{\varepsilon}(u, u)\right)^{\frac{1}{2}}, u \in V$. We remark that there exist positive numbers $\tilde{\varepsilon}_{0}, \tilde{C}_{0}, \tilde{C}_{0}$ such that, for $0<\varepsilon \leqq \tilde{\varepsilon}_{0}$,

$$
\tilde{c}_{0}\|u\|_{H_{\varepsilon}^{1}(Q)} \leqq\|u\|_{V_{\varepsilon}} \leqq \tilde{C}_{0}\|u\|_{H_{\varepsilon}^{1}(Q)}, \quad u \in V_{\varepsilon} .
$$

The inequalities $(2.4)_{\varepsilon}$ are a direct consequence of the hypotheses imposed on $g_{i}$, $i=1,2$, and on the Poincaré inequalities on $Q^{i}, i=1,2$.

To define the limit problem for $\varepsilon=0$, we let $H_{0}=L^{2}((0,1)) \times L^{2}((0,1))$ with the inner product

$$
(\xi, \zeta)_{H_{0}}=\int_{0}^{1} g_{1} \xi_{1} \zeta_{1} d x_{1}+\int_{0}^{1} g_{2} \xi_{2} \zeta_{2} d x_{2}
$$

and let

$$
\mathscr{V}_{0}=\left\{\xi=\left(\xi_{1}, \xi_{2}\right) \in H^{1}((0,1)) \times H^{1}((0,1)): \xi_{1}(1)=\xi_{2}(1)=0, \xi_{1}(0)=\xi_{2}(0)\right\} .
$$

For $v=\left(v_{1}, v_{2}\right) \in \mathscr{V}_{0}, \xi=\left(\xi_{1}, \xi_{2}\right) \in \mathscr{V}_{0}$, we set

$$
a_{0}(v, \xi)=\int_{0}^{1} g_{1} v_{1 x_{1}} \xi_{1 x_{1}} d x_{1}+\int_{0}^{1} g_{2} v_{2 x_{2}} \xi_{2 x_{2}} d x_{2},
$$

and we equip $\mathscr{V}_{0}$ with the norm $\|\cdot\|_{\mathscr{\gamma}_{0}}$ defined by $\|u\|_{\mathscr{r}_{0}}=\left(a_{0}(v, v)\right)^{\frac{1}{2}}$. Actually, we can choose the constants $\tilde{c}_{0}$ and $\tilde{C}_{0}$ in $(2.4)_{\varepsilon}$ so that

$$
\tilde{\mathcal{C}}_{0}\|v\|_{H^{1}((0,1)) \times H^{1}((0,1))} \leqq\|v\|_{\mathscr{r}_{0}} \leqq \tilde{C}_{0}\|v\|_{H^{1}((0,1)) \times H^{1}((0,1))}
$$

for all $v=\left(v_{1}, v_{2}\right) \in H^{1}((0,1)) \times H^{1}((0,1))$ with $v_{1}(1)=v_{2}(1)=0$.

If we also let $G_{10}\left(x_{1}\right)=G\left(x_{1}, 0\right), G_{20}\left(x_{2}\right)=G\left(0, x_{2}\right), \hat{G}_{0}=\left(G_{10}, G_{20}\right)$, then the limit variational problem is to find a function $v \in \mathscr{V}_{0}$ such that, for $\xi \in \mathscr{V}_{0}$, we have

$$
\left(v_{t}, \xi\right)_{H_{0}}+a_{0}(v, \xi)=-\left(\hat{f}(v)+\hat{G}_{0}, \xi\right)_{H_{0}} .
$$

This problem is equivalent to finding a $v=\left(v_{1}, v_{2}\right) \in \mathscr{V}_{0}$ such that

$$
\begin{gathered}
v_{1 t}-\frac{1}{g_{1}}\left(g_{1} v_{1 x_{1}}\right)_{x_{1}}=-f\left(v_{1}\right)-G_{10} \quad \text { in }(0,1), \\
v_{2 t}-\frac{1}{g_{2}}\left(g_{2} v_{2 x_{2}}\right)_{x_{2}}=-f\left(v_{2}\right)-G_{20} \quad \text { in }(0,1), \\
v_{1}(1, t)=v_{2}(1, t)=0, \quad v_{1}(0, t)=v_{2}(0, t), \\
g_{1}(0) v_{1 x_{1}}(0, t)+g_{2}(0) v_{2 x_{2}}(0, t)=0 .
\end{gathered}
$$

REMARK 2.1. In the special case where $g_{1}(0)=g_{2}(0)$ and $g_{1 x_{1}}(0)=g_{2 x_{2}}(0)$, it is easy to show that $(\mathrm{P})_{0}$ is equivalent to an equation on a line. In fact, if $v=\left(v_{1}, v_{2}\right)$ is a 
solution of $(\mathrm{P})_{0}$, and we set

$$
\begin{gathered}
v^{*}(s, t)=\left\{\begin{array}{lr}
v_{1}(s, t), & 0 \leqq s \leqq 1, \\
v_{2}(-s, t), & -1 \leqq s \leqq 0,
\end{array} g^{*}(s)= \begin{cases}g_{1}(s), & 0 \leqq s \leqq 1, \\
g_{2}(-s), & -1 \leqq s \leqq 0,\end{cases} \right. \\
G^{*}(s)= \begin{cases}G_{10}(s), & 0 \leqq s \leqq 1, \\
G_{20}(-s), & -1 \leqq s \leqq 0,\end{cases}
\end{gathered}
$$

then $v^{*}$ satisfies the equation

$(\mathrm{Q})^{*}$

$$
\begin{gathered}
v_{t}^{*}-\frac{1}{g^{*}}\left(g^{*} v_{s}^{*}\right)_{s}=-f\left(v^{*}\right)-G^{*} \quad \text { in }(-1,1), \\
v^{*}(-1, t)=v^{*}(1, t)=0 .
\end{gathered}
$$

Also, if $g_{1}=1$, then $g^{*}=1$.

Now let $Q_{\varepsilon}=\left\{\left(\tilde{x}_{1}, \tilde{x}_{2}\right): 0<\tilde{x}_{2}<\varepsilon g^{*}\left(\tilde{x}_{1}\right),-1<\tilde{x}_{1}<1\right\}, \quad \Gamma_{\varepsilon}^{0}=\partial Q_{\varepsilon} \cap\left\{\tilde{x}_{1}=-1\right\}$, $\Gamma_{\varepsilon}^{1}=\partial Q_{\varepsilon} \cap\left\{\tilde{x}_{1}=1\right\}$. In [13], it was proved that the limit problem at $\varepsilon=0$ of the reaction-diffusion equation

$$
\begin{array}{ll}
u_{t}-\Delta u=-f(u)-G & \text { in } Q_{\varepsilon}, \\
u=0 & \text { in } \Gamma_{\varepsilon}^{0} \cup \Gamma_{\varepsilon}^{1}, \\
\frac{\partial u}{\partial n_{\varepsilon}}=0 & \text { in } \partial Q_{\varepsilon} \backslash\left(\Gamma_{\varepsilon}^{0} \cup \Gamma_{\varepsilon}^{1}\right)
\end{array}
$$

is the problem $(\mathrm{Q})^{*}$. This means that, if the L-shaped domain has the property $g_{1}(0)=g_{2}(0), g_{1 x_{1}}(0)=g_{2 x_{2}}(0)$, then the limit problem for $\varepsilon=0$ is the same as that for a thin domain over a line segment defined by the function $g^{*}$ above.

For any $\hat{h}=\left(h_{1}, h_{2}\right) \in \mathscr{H}_{\varepsilon}$, we consider the problem: find $u=\left(u_{1}, u_{2}\right) \in \mathscr{V}_{\varepsilon}$ such that

$$
a_{\varepsilon}(u, w)=(\hat{h}, w), \quad \text { for all } w \in \mathscr{V}_{\varepsilon} .
$$

Going back to the domain $Q_{\varepsilon}$ and applying the Lax-Milgram Theorem, one shows that, for any $\hat{h} \in \mathscr{H}_{\varepsilon}$, there is a unique solution $u$ of the problem $(2.5)_{\varepsilon}$. Moreover, using $(2.4)_{\varepsilon}$, one proves that, for $0<\varepsilon \leqq \tilde{\varepsilon}_{0}$,

$$
\|u\|_{H_{\varepsilon}^{1}(Q)} \leqq c\|\hat{h}\|_{H_{\varepsilon}} .
$$

Likewise, for any $\hat{h}_{0}=\left(h_{10}, h_{20}\right) \in H_{0}$, we consider the problem: find $v=\left(v_{1}, v_{2}\right) \in \mathscr{V}_{0}$ such that

$$
a_{0}(v, \xi)=\left(\hat{h}_{0}, \xi\right), \text { for all } \xi \in \mathscr{V}_{0} .
$$

Thanks to the Lax-Milgram Theorem, for any $\hat{h}_{0} \in H_{0}$, there is a unique solution $v$ of the problem $(2.5)_{0}$. Moreover, due to $(2.4)_{0}$, we have

$$
\|v\|_{r_{0}} \leqq c\left\|\hat{h}_{0}\right\|_{H_{0}} .
$$

Writing the differential equations satisfied by $v_{1}, v_{2}$, and using the fact that the functions $v_{1}, v_{2}$ are functions of one real variable only, one shows in addition that

$$
\|v\|_{H^{2}((0,1)) \times H^{2}((0,1))} \leqq c\left\|\hat{h}_{0}\right\|_{H_{0}} .
$$

The triple $\left\{\mathscr{V}_{0}, H_{0}, a_{0}(\cdot, \cdot)\right\}$ (respectively $\left\{\mathscr{V}_{\varepsilon}, \mathscr{H}_{\varepsilon}, a_{\varepsilon}(\cdot, \cdot)\right\}$ ) defines a unique unbounded 
operator $A_{0}$ on $\mathscr{V}_{0}$ (respectively $A_{\varepsilon}$ on $\mathscr{V}_{\varepsilon}$ ) with domain $D\left(A_{0}\right)$ (respectively $D\left(A_{\varepsilon}\right)$ ) in the following way: an element $v \in \mathscr{V}_{0}$ (respectively $u \in \mathscr{V}_{\varepsilon}$ ) belongs to $D\left(A_{0}\right)$ (respectively $\left.D\left(A_{\varepsilon}\right)\right)$ if the form

$$
\left.\xi \mapsto a_{0}(v, \xi) \quad \text { (respectively } w \mapsto a_{\varepsilon}(u, w)\right)
$$

is continuous on $\mathscr{V}_{0}$ (respectively $\mathscr{V}_{\varepsilon}$ ) for the topology induced by $H_{0}$ (respectively $\mathscr{H}_{\varepsilon}$ ). Then $a_{0}(v, \xi)=\left(A_{0} v, \xi\right)_{H_{0}}, A_{0} v \in H_{0}$, which defines $A_{0}$ (respectively $a_{\varepsilon}(u, w)=$ $\left(\mathrm{A}_{\varepsilon} \mathrm{u}, \mathrm{w}\right)_{H_{\varepsilon}}, A_{\varepsilon} u \in \mathscr{H}_{\varepsilon}$, which defines $A_{\varepsilon}$ ). The operator $A_{0}$ (respectively $A_{\varepsilon}$ ) is selfadjoint on $H_{0}$ (respectively $\mathscr{H}_{\varepsilon}$ ), is positive and is sectorial in the sense of [19]. Moreover, $D\left(A_{0}^{\frac{1}{2}}\right)=\mathscr{V}_{0}$ (respectively $D\left(A_{\varepsilon}^{\frac{1}{1}}\right)=\mathscr{V}_{\varepsilon}$ ). Arguing as in [19, Section 5.3], and using the hypotheses $(2.1),(2.2)$, one shows that $(\tilde{\mathrm{P}})_{\varepsilon}$ generates a $C^{0}$-semigroup $T_{\varepsilon}(t)$ on $\mathscr{V}_{\varepsilon}$. Arguing as in [10, Section 4.3] (see also [13, Section 2]), one shows that $(\tilde{\mathrm{P}})_{\varepsilon}$ is a gradient system and has a connected global attractor $\mathscr{A}_{\varepsilon}$. Moreover, $\mathscr{A}_{\varepsilon}$ is the unstable manifold of the set $E_{\varepsilon}$ of the equilibrium points of $(\tilde{\mathrm{P}})_{\varepsilon}$. We recall that $\mathscr{A}_{\varepsilon}$ is the global attractor of the semigroup $T_{\varepsilon}(t)$ if $\mathscr{A}_{\varepsilon}$ is a compact subset of $\mathscr{V}_{\varepsilon}, \mathscr{A}_{\varepsilon}$ is invariant (that is, $T_{\varepsilon}(t) \mathscr{A}_{\varepsilon}=\mathscr{A}_{\varepsilon}$ for $t \geqq 0$ ), $\mathscr{A}_{\varepsilon}$ attracts every bounded set $B$ of $\mathscr{V}_{k}$; that is, for each bounded set $B$, for each $\eta>0$, there exists a time $\tau=\tau(B, \eta)$ such that, for $t \geqq \tau, T_{\varepsilon}(B)$ is contained in the $\eta$-neighbourhood $\mathscr{N}_{\gamma_{\varepsilon}}\left(\mathscr{A}_{\varepsilon}, \eta\right)$ of $\mathscr{A}_{\varepsilon}$ in $\mathscr{V}_{\varepsilon}$. One shows also that the problem $(\tilde{\mathrm{P}})_{0}$ generates a $C^{0}$-semigroup $T_{0}(t)$ on $\mathscr{V}_{0}$ which is compact for $t>0$ and has a global attractor $\mathscr{A}_{0}$ in $\mathscr{V}_{0}$. Local existence of solutions of $(\mathrm{P})_{0}$ follows standard procedures. We remark only about the manner in which the boundary and compatibility conditions in $(\mathrm{P})_{0}$ lead to the global existence of solutions and the existence of the global attractor. For $v=\left(v_{1}, v_{2}\right) \in \mathscr{V}_{0}$, if $V\left(v_{1}, v_{2}\right)=V_{1}\left(v_{1}\right)+V_{2}\left(v_{2}\right)$ is the energy for $(\mathbf{P})_{0}$, where

$$
V_{j}\left(v_{j}\right)=\int_{0}^{1} g_{j}\left[\frac{1}{2}\left(v_{j x_{j}}\right)^{2}+F\left(v_{j}\right)+G_{j 0} v_{j}\right]
$$

and $F(s)=\int_{0}^{s} f$, then the derivative $\dot{V}\left(v_{1}, v_{2}\right)$ along the solutions of $(\mathrm{P})_{0}$ is easily seen to satisfy $V\left(v_{1}, v_{2}\right)=-\int_{0}^{1}\left(g_{1} v_{1 \mathrm{t}}^{2}+g_{2} v_{2 \mathrm{t}}^{2}\right)$. The function $V\left(v_{1}, v_{2}\right)$ is a Lyapunov function and serves as an equivalent norm in $\mathscr{V}_{0}$. This gives global existence of solutions and defines the semigroup $T_{0}(t)$. Obviously, $T_{0}(t)$ is compact for $t>0$. Also, the $\omega$-limit set of any solution must belong to the set of equilibrium points. Since the set of equilibrium points is bounded, it follows that the global attractor exists (see, for example, [10]).

We can consider the attractor $\mathscr{A}_{0}$ as a subset of $H_{\varepsilon}^{1}(Q)$. Our main result is now stated in the following theorem. If $Z$ is a Banach space and $B_{1}, B_{2}$ are two subsets of $Z$, we set

$$
\delta_{Z}\left(B_{1}, B_{2}\right)=\sup _{b_{1} \in B_{1}} \inf _{b_{2} \in B_{2}}\left\|b_{1}-b_{2}\right\|_{Z}
$$

THEOREM 2.2. The attractors $\mathscr{A}_{\varepsilon}, 0 \leqq \varepsilon \leqq \varepsilon_{0}$, are upper semicontinuous at $\varepsilon=0$; that is,

$$
\delta_{H_{\varepsilon}^{1}(Q)}\left(\mathscr{A}_{\varepsilon}, \mathscr{A}_{0}\right) \rightarrow 0 \text { as } \varepsilon \rightarrow 0 \text {. }
$$

We also give an estimate on the distance between the solutions of the problems $(\tilde{\mathrm{P}})_{\varepsilon}$ and $(\tilde{\mathrm{P}})_{0}$ on any finite time interval.

In spirit, the proof of Theorem 2.2 follows along the lines in [13] for a parabolic equation on a thin domain. However, there are several important differences. For PDE on a thin domain $Q_{\varepsilon}$ over a line segment defined by $Q_{\varepsilon}=$ 
$\left\{\left(\tilde{x}_{1}, \tilde{x}_{2}\right): 0<\tilde{x}_{2}<\varepsilon g\left(\tilde{x}_{1}\right), 0<\tilde{x}_{1}<1\right\}$, the first step of the analysis is to map $Q_{\varepsilon}$ onto the canonical domain $Q=(0,1) \times(0,1)$. In this case, there is a natural embedding of the solution space for the limit equation on the line into the solution space for the perturbed equation on $Q$. Also, there is a natural projection in the opposite direction using a restricted mean value operator which takes a function $u$ on the square $Q$ to the function $(M u)\left(x_{1}\right)=\int_{0}^{1} u\left(x_{1}, x_{2}\right) d x_{2}$ on the line segment $[0,1]$. In the case of L-shaped domains, neither of these properties is true because of the junction region. Therefore, we must introduce an operator $I_{\varepsilon}$ which will map $\mathscr{V}_{0}$ into $\mathscr{V}_{\varepsilon}$ and an operator $M_{\varepsilon}$ which maps $\mathscr{V}_{\varepsilon}$ into $\mathscr{V}_{0}$. The operator $I_{\varepsilon}$ will be approximately the identity, while the operator $M_{\varepsilon}$ will be approximately a restricted mean value operator (see Section 3 and the proof of Theorem 2.2). The necessity for the introduction of these operators $I_{\varepsilon}$ and $M_{\varepsilon}$ makes the estimates needed in the proof of Theorem 2.2 much more complicated. We remark that the precise estimates of the convergence of the attractors $\mathscr{A}_{\varepsilon}$ to the attractor $\mathscr{A}_{0}$ will depend upon the manner in which the operators $I_{\varepsilon}$ and $M_{\varepsilon}$ converge respectively to the identity and the restricted mean value operator.

We present the outline of the proof to bring out the essential difficulties. We first remark that, throughout the paper, $C$ (respectively $k(r)$ ) will denote a generic positive constant (respectively a generic function of $r$ ) independent of $\varepsilon$. Using the properties (2.1), (2.2), as well as regularity properties of $T_{\varepsilon}(t)$ and $T_{0}(t)$, and the fact that $T_{\varepsilon}(t)$ is a gradient system, it is possible to proceed as in [13] to obtain the following result:

LEMMA 2.3. There is a positive constant $C_{0}$ such that, for $0<\varepsilon \leqq \varepsilon_{0}$,

$$
\left\|\varphi^{\varepsilon}\right\|_{\mathscr{r}_{\varepsilon}} \leqq C_{0}, \text { for all } \varphi^{\varepsilon} \in \mathscr{A}_{\varepsilon} \text {. }
$$

Moreover, for any $r_{0}>0$, there is a positive constant $k\left(r_{0}\right)$ such that, for $0<\varepsilon \leqq \varepsilon_{0}$, and any $u_{0} \in \mathscr{V}_{\varepsilon},\left\|u_{0}\right\|_{r_{\varepsilon}} \leqq r_{0}, u^{\varepsilon}=T_{\varepsilon}(t) u_{0}$ satisfies

$$
\begin{gathered}
\left\|u^{\varepsilon}(t)\right\|_{\mathscr{V}_{\varepsilon}} \leqq k\left(r_{0}\right), \quad t \geqq 0, \\
\int_{0}^{t}\left\|\frac{d}{d s}\left(s u_{s}^{\varepsilon}\right)\right\|_{H_{\varepsilon}}^{2} d s+\left\|t u_{t}^{\varepsilon}\right\|_{\mathscr{V}_{\varepsilon}}^{2} \leqq k\left(r_{0}\right)\left(1+t^{2}\right), \\
\int_{0}^{t}\left\|u_{t}^{\varepsilon}\right\|_{H_{\varepsilon}}^{2} d s \leqq k\left(r_{0}\right), \quad t \geqq 0 .
\end{gathered}
$$

Finally, if $v(t)=T_{0}(t) v_{0}$ with $\left\|v_{0}\right\|_{\mathscr{r}_{0}} \leqq r_{0}$, then

$$
\begin{gathered}
\|v\|_{H^{1}((0,1)) \times H^{1}((0,1))} \leqq k\left(r_{0}\right), \quad t \geqq 0, \\
\int_{0}^{t}\left\|v_{t}\right\|_{L^{2}((0,1)) \times L^{2}((0,1))}^{2} d s \leqq k\left(r_{0}\right), \\
\int_{0}^{t}\left\|\frac{d}{d s}\left(s v_{s}\right)\right\|_{L^{2}((0,1)) \times L^{2}((0,1))}^{2} d s+\left\|t v_{t}\right\|_{H^{1}((0,1)) \times H^{1}((0,1))}^{2} \leqq k\left(r_{0}\right)\left(1+t^{2}\right), \\
\int_{0}^{t}\left\|\frac{d}{d s}(s v)\right\|_{H^{2}((0,1)) \times H^{2}((0,1))}^{2} d s+\|t v\|_{H^{2}((0,1)) \times H^{2}((0,1))}^{2} \leqq k\left(r_{0}\right)\left(1+t^{2}\right), \\
\int_{0}^{t}\|v\|_{H^{2}((0,1)) \times H^{2}((0,1))}^{2} d s \leqq k\left(r_{0}\right)(1+t),
\end{gathered}
$$


for $t \geqq 0$.

Proof of Theorem 2.2. Since $\mathscr{A}_{0}$ is the global attractor for $(\tilde{\mathrm{P}})_{0}$, for any $\eta>0$ and any $r_{1}>0$, there exists a positive number $\tau_{\eta}$ such that, for $t \geqq \tau_{\eta}$, for $\left\|v_{0}\right\|_{H^{1}((0,1)) \times H^{1}((0,1))} \leqq r_{1}$,

$$
\delta_{\mathscr{r}_{0}}\left(T_{0}(t) v_{0}, \mathscr{A}_{0}\right) \leqq \frac{\eta}{2} .
$$

We construct a linear mapping $I_{\varepsilon}: \mathscr{V}_{0} \rightarrow \mathscr{V}_{\varepsilon}$ with the property that it is the identity on a subset of $\tilde{Q}_{\varepsilon}$ of $(0,1)^{2} \times(0,1)^{2}$ with the measure of $(0,1)^{2} \times(0,1)^{2} \backslash \tilde{Q}_{\varepsilon}$ approaching zero as $\varepsilon \rightarrow 0$ and such that there exists a positive constant $C$ such that, for all $\xi \in \mathscr{V}_{0}$,

$$
\left\|I_{\varepsilon} \xi\right\|_{\mathscr{r}_{\varepsilon}} \leqq C\|\xi\|_{\mathscr{r}_{0}}, \quad\left\|\xi-I_{\varepsilon} \xi\right\|_{H_{\varepsilon}} \leqq C \varepsilon\|\xi\|_{H^{1}((0,1)) \times H^{1}((0,1))} .
$$

We also construct a linear mapping $M_{\varepsilon}: \mathscr{V}_{\varepsilon} \rightarrow \mathscr{V}_{0}$ with the property that, on a subset of approximately full measure, it is the restricted mean value with respect to $x_{2}$ on $Q^{1}$ and with respect to $x_{1}$ on $Q^{2}$ and such that there exists a positive constant $C$ such that, for all $u \in \mathscr{W}_{\varepsilon}$,

$$
\left\|M_{\varepsilon} u\right\|_{\mathscr{V}_{0}} \leqq C\|u\|_{\mathscr{V}_{\varepsilon}}, \quad\left\|u-M_{\varepsilon} u\right\|_{L^{2}(Q)} \leqq C \varepsilon\|u\|_{\mathscr{V}_{\varepsilon}} .
$$

We then prove that, for any $r_{0}>0$, there is a positive constant $k\left(r_{0}\right)$ such that for $\left\|u_{0}\right\|_{\mathscr{r}_{\varepsilon}} \leqq r_{0}$, for $t \geqq 0$, we have

$$
\left\|t T_{0}(t) M_{\varepsilon} u_{0}-t T_{\varepsilon}(t) u_{0}\right\|_{H_{\varepsilon}^{1}(Q)}^{2} \leqq \varepsilon k\left(r_{0}\right) e^{k\left(r_{0}\right) t} .
$$

By (2.9) and (2.16), $\left\|M_{\varepsilon} \varphi_{\varepsilon}\right\|_{r_{0}} \leqq C C_{0}$; we set $r_{1}=C C_{0}$. From (2.17), we deduce that, for $0<t \leqq \tau_{\eta}$, for $\varphi_{\varepsilon} \in \mathscr{A}_{\varepsilon}$,

$$
\left\|t T_{0}(t) M_{\varepsilon} \varphi_{\varepsilon}-t T_{\varepsilon}(t) \varphi_{\varepsilon}\right\|_{H_{\varepsilon}^{1}(Q)}^{2} \leqq \varepsilon k\left(C_{0}\right) e^{k\left(C_{0}\right) \tau_{n}}
$$

We now choose $\varepsilon_{0}$ so that

$$
\frac{1}{\tau_{\eta}} \varepsilon_{0} k\left(C_{0}\right) e^{k\left(C_{0}\right) r_{\eta}} \leqq \frac{\eta}{2} .
$$

From (2.14) and (2.18), we conclude that $\delta_{H_{\varepsilon}^{1}(Q)}\left(T_{\varepsilon}\left(\tau_{\eta}\right) \varphi_{\varepsilon}, \mathscr{A}_{0}\right) \leqq \eta$. By the invariance of $\mathscr{A}_{\varepsilon}$, this implies that $\delta_{H_{\varepsilon}^{1}(Q)}\left(\mathscr{A}_{\varepsilon}, \mathscr{A}_{0}\right) \leqq \eta$ and the theorem is proved.

The next few sections are devoted to introducing the operators $I_{\varepsilon}$ and $M_{\varepsilon}$ and proving the inequality $(2.17)$.

\section{The mappings $I_{\varepsilon}$ and $M_{\varepsilon}$}

If $x_{1}\left(\varepsilon, x_{2}\right)$ and $x_{2}\left(\varepsilon, x_{1}\right)$ are as defined in Section 2 (see (2.3)), we let

$$
\begin{array}{ll}
x_{1 \varepsilon M}=\max _{0 \leqq x_{2} \leqq 1} x_{1}\left(\varepsilon, x_{2}\right), & x_{1 \varepsilon m}=\min _{0 \leqq x_{2} \leqq 1} x_{1}\left(\varepsilon, x_{2}\right), \\
x_{2 \varepsilon M}=\max _{0 \leqq x_{1} \leqq 1} x_{2}\left(\varepsilon, x_{1}\right), & x_{2 \varepsilon m}=\min _{0 \leqq x_{1} \leqq 1} x_{2}\left(\varepsilon, x_{1}\right) .
\end{array}
$$

From the Mean Value Theorem and (2.3), we deduce that

$$
\begin{aligned}
& \left|x_{1 \varepsilon M}-x_{1 e m}\right| \leqq C \varepsilon^{2}, \\
& \left|x_{2 \varepsilon M}-x_{2 \varepsilon m}\right| \leqq C \varepsilon^{2} .
\end{aligned}
$$


For $n=1,2$, we define $Q_{n \varepsilon M}=Q_{n \varepsilon M}^{1} \times Q_{n \varepsilon M}^{2}$, where

$$
Q_{n \varepsilon M}^{1}=\left(0, n x_{1 \varepsilon M}\right) \times(0,1), \quad Q_{n \varepsilon M}^{2}=(0,1) \times\left(0, n x_{2 \varepsilon M}\right),
$$

and let $H_{n \varepsilon M} \simeq L^{2}\left(Q_{n \varepsilon M}\right)$ be the Banach space of functions on $Q_{n \varepsilon M}$ with the norm $\|\cdot\|_{H_{n s} M}$ induced by the inner product

$$
(\xi, \zeta)_{H_{n \varepsilon M}}=\int_{Q_{n \varepsilon M}^{1}} g_{1} \xi_{1} \zeta_{1} d x_{1} d x_{2}+\int_{Q_{n E M}^{2}} g_{2} \xi_{2} \zeta_{2} d x_{1} d x_{2} .
$$

We also let $H_{n \varepsilon M}^{1}=H^{1}\left(Q_{n \varepsilon M}\right)$.

For any $v=\left(v_{1}, v_{2}\right) \in \mathscr{V}_{0}$, we define $I_{\varepsilon} v=\left(I_{\varepsilon}^{1} v_{1}, I_{\varepsilon}^{2} v_{2}\right)$ by the relation

$$
\begin{aligned}
& I_{\varepsilon}^{1} v_{1}\left(x_{1}, x_{2}\right)= \begin{cases}v_{1}\left(x_{1}\right) & x_{1} \in\left(2 x_{1 \varepsilon M}, 1\right], \\
v_{1}\left(2\left(x_{1}-x_{1 \varepsilon M}\right)\right) & x_{1} \in\left(x_{1 \varepsilon M}, 2 x_{1 \varepsilon M}\right], \\
v_{1}(0) & x_{1} \in\left[0, x_{1 \varepsilon M}\right] ;\end{cases} \\
& I_{\varepsilon}^{2} v_{2}\left(x_{1}, x_{2}\right)= \begin{cases}v_{2}\left(x_{2}\right) & x_{2} \in\left(2 x_{2 \varepsilon M}, 1\right], \\
v_{2}\left(2\left(x_{2}-x_{2 \varepsilon M}\right)\right) & x_{2} \in\left(x_{2 \varepsilon M}, 2 x_{2 \varepsilon M}\right], \\
v_{2}(0) & x_{2} \in\left[0, x_{2 \varepsilon M}\right] .\end{cases}
\end{aligned}
$$

It is clear that $I_{\varepsilon} v \in \mathscr{V}_{\varepsilon} \cap \mathscr{V}_{0}$. Also, there is a positive constant $C$ such that, for $0<\varepsilon \leqq \varepsilon_{0}$ and all $v \in \mathscr{V}_{0}$,

$$
\begin{aligned}
\sup \left\{\left\|v-I_{\varepsilon} v\right\|_{H_{\varepsilon}},\left\|v-I_{\varepsilon} v\right\|_{L^{2}(Q)}\right\} & \leqq C \varepsilon\left(\sum_{i=1}^{2} \int_{0}^{2 x_{i \varepsilon M}} v_{i x_{i}}^{2} d x_{i}\right)^{\frac{1}{2}} \\
& \leqq C \varepsilon\|v\|_{H^{1}((0,1)) \times H^{1}((0,1))},
\end{aligned}
$$

and, for all $v \in \mathscr{V}_{0} \cap\left(H^{2}((0,1)) \times H^{2}((0,1))\right)$,

$$
\sup \left\{\left\|v-I_{\varepsilon} v\right\|_{H_{\varepsilon}},\left\|v-I_{\varepsilon} v\right\|_{L^{2}(Q)}\right\} \leqq C \varepsilon^{1+\frac{1}{2}}\|v\|_{H^{2}((0,1)) \times H^{2}((0,1))},
$$

and

$$
\sup \left\{\left\|v-I_{\varepsilon} v\right\|_{H_{\varepsilon}^{1}(Q)},\left\|v-I_{\varepsilon} v\right\|_{H^{1}((0,1)) \times H^{1}((0,1))}\right\} \leqq C \varepsilon^{\frac{1}{2}}\|v\|_{H^{2}((0,1)) \times H^{2}((0,1))} .
$$

Also,

$$
\left\|I_{\varepsilon} v\right\|_{L^{2}(Q)} \leqq C\|v\|_{L^{2}((0,1)) \times L^{2}((0,1)),}, \quad\left\|I_{\varepsilon} v\right\|_{\mathscr{V}_{\varepsilon}} \leqq C\|v\|_{H^{1}((0,1)) \times H^{1}((0,1))} .
$$

To prove (3.3), we observe

$$
v_{1}-I_{\varepsilon}^{1} v_{1}= \begin{cases}0 & x_{1} \in\left(2 x_{1 \varepsilon M}, 1\right], \\ \int_{0}^{1} v_{1 x_{1}}\left(x_{1}+s\left(x_{1}-2 x_{1 \varepsilon M}\right)\right)\left(2 x_{1 \varepsilon M}-x_{1}\right) d s & x_{1} \in\left(x_{1 \varepsilon M}, 2 x_{1 \varepsilon M}\right], \\ \int_{0}^{1} v_{1 x_{1}}\left(s x_{1}\right) x_{1} d s & x_{1} \in\left[0, x_{1 \varepsilon M}\right],\end{cases}
$$

with a similar expression for $v_{2}-I_{\varepsilon}^{2} v_{2}$. The estimate (3.3a) is now immediate. The estimate (3.3b) is a direct consequence of (3.3a) and the continuous embedding 
of $H^{2}((0,1))$ into $W^{1, \infty}((0,1))$. To prove the estimate $(3.3 \mathrm{c})$, we remark that, for $i=1,2$,

$$
\begin{aligned}
\int_{0}^{1}\left(v_{i x_{i}}-\left(I_{\varepsilon}^{i} v_{i}\right)_{x_{i}}\right)^{2} d x_{i} & =\int_{0}^{x_{i \varepsilon M}} v_{i x_{i}}^{2}\left(x_{i}\right) d x_{i}+\int_{x_{i \varepsilon M}}^{2 x_{i \varepsilon M}}\left(v_{i x_{i}}\left(x_{i}\right)-2 v_{i x_{i}}\left(2\left(x_{i}-x_{i \varepsilon M}\right)\right)\right)^{2} d x_{i} \\
& \leqq 4 x_{i \varepsilon M} \max _{0 \leqq x_{i} \leqq 1} v_{i x_{i}}^{2} \leqq C \varepsilon\left\|v_{i}\right\|_{H^{2}((0,1))}^{2},
\end{aligned}
$$

which, together with (3.3a), implies (3.3c). The first estimate in (3.4) is obvious and the second one follows by differentiating $I_{\varepsilon} v$.

To define the operator $M_{\varepsilon}: \mathscr{V}_{\varepsilon} \rightarrow \mathscr{V}_{0}$, we need some additional notation. For any function $\xi \in L^{2}((0,1) \times(0,1))$, we define partial averages by the relations

$$
m_{1} \xi\left(x_{1}\right)=\int_{0}^{1} \xi\left(x_{1}, x_{2}\right) d x_{2}, \quad m_{2} \xi\left(x_{2}\right)=\int_{0}^{1} \xi\left(x_{1}, x_{2}\right) d x_{1}
$$

and, for $u_{j} \in L^{2}\left(Q^{j}\right), j=1,2$, the "average" of $u_{j}$ over $J_{\varepsilon}^{j}$ by the relations

$$
\begin{aligned}
& \bar{m}_{\varepsilon}^{1} u_{1}=\frac{1}{\int_{J_{\varepsilon}^{1}} g_{1}\left(x_{1}\right) d x_{1} d x_{2}} \int_{J_{\varepsilon}^{1}} g_{1}\left(x_{1}\right) u_{1}\left(x_{1}, x_{2}\right) d x_{1} d x_{2}, \\
& \bar{m}_{\varepsilon}^{2} u_{2}=\frac{1}{\int_{J_{\varepsilon}^{2}} g_{2}\left(x_{2}\right) d x_{1} d x_{2}} \int_{J_{\varepsilon}^{2}} g_{2}\left(x_{2}\right) u_{2}\left(x_{1}, x_{2}\right) d x_{1} d x_{2} .
\end{aligned}
$$

We now define the operator $M_{\varepsilon} u=\left(M_{\varepsilon}^{1} u_{1}, M_{\varepsilon}^{2} u_{2}\right)$ for $u \in \mathscr{V}_{\varepsilon}$ by the relations

$$
\begin{gathered}
M_{\varepsilon}^{1} u_{1}= \begin{cases}m_{1} u_{1}\left(x_{1}\right) & x_{1} \in\left(2 x_{1 \varepsilon M}, 1\right], \\
\frac{x_{1}-x_{1 \varepsilon M}}{x_{1 \varepsilon M}} m_{1} u_{1}\left(x_{1}\right)+\frac{2 x_{1 \varepsilon M}-x_{1}}{x_{1 \varepsilon M}} \bar{m}_{\varepsilon}^{1} u_{1} & x_{1} \in\left(x_{1 \varepsilon M}, 2 x_{1 \varepsilon M}\right], \\
\bar{m}_{\varepsilon}^{1} u_{1} & x_{1} \in\left[0, x_{1 \varepsilon M}\right] ;\end{cases} \\
M_{\varepsilon}^{2} u_{2}= \begin{cases}m_{2} u_{2}\left(x_{2}\right) & x_{2} \in\left(2 x_{2 \varepsilon M}, 1\right], \\
\frac{x_{2}-x_{2 \varepsilon M}}{x_{2 \varepsilon M}} m_{2} u_{2}\left(x_{2}\right)+\frac{2 x_{2 \varepsilon M}-x_{2}}{x_{2 \varepsilon M}} \bar{m}_{\varepsilon}^{2} u_{2} & x_{2} \in\left(x_{2 \varepsilon M}, 2 x_{2 \varepsilon M}\right], \\
\bar{m}_{\varepsilon}^{2} u_{2} & x_{2} \in\left[0, x_{2 \varepsilon M}\right] ;\end{cases}
\end{gathered}
$$

It is clear that $M_{\varepsilon}: \mathscr{V}_{\varepsilon} \rightarrow \mathscr{V}_{0}$, since

$$
\bar{m}_{\varepsilon}^{1} u_{1}=\bar{m}_{\varepsilon}^{2} u_{2}=\left(\int_{J_{\varepsilon}} d \tilde{x}_{1} d \tilde{x}_{2}\right)^{-1} \int_{J_{\varepsilon}} u\left(\tilde{x}_{1}, \tilde{x}_{2}\right) d \tilde{x}_{1} d \tilde{x}_{2} .
$$


We also remark that $M_{\varepsilon}: \mathscr{V}_{\varepsilon} \rightarrow \mathscr{V}_{\varepsilon}$. We want to prove that there is a positive constant $C$ such that, for $0<\varepsilon \leqq \varepsilon_{0}$ and all $u \in \mathscr{V}_{\varepsilon}$, we have

$$
\left.\begin{array}{c}
\left\|u-M_{\varepsilon} u\right\|_{H_{\varepsilon}} \leqq C \varepsilon\|u\|_{V_{\varepsilon}}, \\
\text { (i) }\left\|M_{\varepsilon} u\right\|_{L^{2}(Q)} \leqq C\|u\|_{L^{2}(Q)}, \\
\text { (ii) }\left\|M_{\varepsilon} u\right\|_{L^{2}\left(Q_{n \varepsilon M}\right)} \leqq C\|u\|_{L^{2}\left(Q_{n \varepsilon M}\right)} \\
\text { (iii) }\left\|M_{\varepsilon} u\right\|_{H^{1}(Q)} \leqq C\|u\|_{V_{\varepsilon} \cdot}
\end{array}\right\}
$$

The estimates (3.7)(i) and (3.7)(ii) are obvious.

To prove (3.6), we observe that, for $x_{1} \in\left[x_{1 \varepsilon M}, 2 x_{1 \varepsilon M}\right]$, the quantities

$$
\theta=\frac{x_{1}-x_{1 \varepsilon M}}{x_{1 \varepsilon M}} \text { and } 1-\theta=\frac{2 x_{1 \varepsilon M}-x_{1}}{x_{1 \varepsilon M}}
$$

are in $[0,1]$. Therefore,

$$
\left\|u_{1}-M_{\varepsilon}^{1} u_{1}\right\|_{L^{2}\left(Q^{1}\right)}^{2} \leqq 2\left\|u_{1}-m_{1} u_{1}\right\|_{L^{2}\left(Q^{1}\right)}^{2}+2\left\|u_{1}-\bar{m}_{\varepsilon}^{1} u_{1}\right\|_{L^{2}\left(Q_{2 \varepsilon M}^{1}\right)}^{2} .
$$

By the Poincaré inequality (see [13], Lemma 3.1]), we have

$$
\left\|u_{1}-m_{1} u_{1}\right\|_{L^{2}\left(Q^{1}\right)}^{2} \leqq C_{1} \varepsilon^{2}\left\|\frac{1}{\varepsilon} u_{1 x_{2}}\right\|_{L^{2}\left(Q^{1}\right)}^{2} \leqq C_{1} \varepsilon^{2}\left\|u_{1}\right\|_{H_{\varepsilon}^{1}(Q)}^{2} .
$$

It remains to estimate the second term of the right-hand side of (3.8). If $u_{1}$ belongs to $C^{\infty}\left(Q_{2 \varepsilon M}^{1}\right)$, we can write

$$
u_{1}\left(x_{1}, x_{2}\right)=u_{1}\left(x_{1}^{0}, x_{2}^{0}\right)+\int_{x_{2}^{0}}^{x_{2}} u_{1 x_{2}}\left(x_{1}^{0}, s_{2}\right) d s_{2}+\int_{x_{1}^{0}}^{x_{1}} u_{1 x_{1}}\left(s_{1}, x_{2}\right) d s_{1},
$$

or, also,

$$
\begin{aligned}
u_{1}\left(x_{1}, x_{2}\right)= & \frac{1}{x_{1 \varepsilon M}} \int_{0}^{1} \int_{0}^{x_{1 \varepsilon M}} u_{1}\left(x_{1}^{0}, x_{2}^{0}\right) d x_{1}^{0} d x_{2}^{0} \\
& +\frac{1}{x_{1 \varepsilon M}}\left[\int_{0}^{x_{1 \varepsilon M}} \int_{x_{1}^{0}}^{x_{1}} u_{1 x_{1}}\left(s_{1}, x_{2}\right) d s_{1} d x_{1}^{0}\right. \\
& \left.+\int_{0}^{1} \int_{0}^{x_{1 \varepsilon M}} \int_{x_{2}^{0}}^{x_{2}} u_{1 x_{2}}\left(x_{1}^{0}, s_{2}\right) d s_{2} d x_{1}^{0} d x_{2}^{0}\right] .
\end{aligned}
$$

Using the equality (3.10), the estimates (2.3), as well as the Cauchy-Schwarz inequality, we prove that

$$
\begin{array}{r}
\left\|u_{1}\left(x_{1}, x_{2}\right)-\frac{1}{x_{1 \varepsilon M}} \int_{0}^{1} \int_{0}^{x_{1 \varepsilon M}} u_{1}\left(s_{1}, s_{2}\right) d s_{1} d s_{2}\right\|_{L^{2}\left(Q_{2 \varepsilon M}^{1}\right)} \\
\leqq C \varepsilon\left[\left\|u_{1 x_{1}}\right\|_{L^{2}\left(Q_{2 \varepsilon}^{1}\right)}+\left\|\frac{1}{\varepsilon} u_{1 x_{2}}\right\|_{L^{2}\left(Q_{2 \varepsilon M}^{1}\right)}\right] .
\end{array}
$$

Likewise, using the equality (3.10), the estimates (2.3) and $\left(2.3^{\prime}\right)$, as well as the 
Cauchy-Schwarz inequality, we show that

$$
\begin{array}{r}
\left\|\bar{m}_{\varepsilon}^{1}\left(u_{1}-\frac{1}{x_{1 \varepsilon M}} \int_{0}^{1} \int_{0}^{x_{1 \varepsilon M}} u_{1}\left(s_{1}, s_{2}\right) d s_{1} d s_{2}\right)\right\|_{L^{2}\left(Q_{2 \varepsilon M}^{1}\right)} \\
\leqq C \varepsilon\left[\left\|u_{1 x_{1}}\right\|_{L^{2}\left(Q_{2 \varepsilon M}^{1}\right)}+\left\|\frac{1}{\varepsilon} u_{1 x_{2}}\right\|_{L^{2}\left(Q_{2 \varepsilon M}^{1}\right)}\right] .
\end{array}
$$

The inequalities (3.11) and (3.12) at once imply that

$$
\left\|u_{1}-\bar{m}_{\varepsilon}^{1} u_{1}\right\|_{L^{2}\left(Q_{2 \varepsilon M}^{1}\right)} \leqq C \varepsilon\left[\left\|u_{1 x_{1}}\right\|_{L^{2}\left(Q_{2 \varepsilon M}^{1}\right)}+\left\|\frac{1}{\varepsilon} u_{1 x_{2}}\right\|_{L^{2}\left(Q_{2 \varepsilon M}^{1}\right)}\right] .
$$

By density of the space $C^{\infty}\left(Q_{2 \varepsilon M}^{1}\right)$ in $H^{1}\left(Q_{2 \varepsilon M}^{1}\right)$, we deduce the above inequality for any $u_{1} \in H^{1}\left(Q_{2 i M}^{1}\right)$. Using similar arguments, we show that the estimates (3.8), (3.9), (3.13) still hold if $i=1$ is replaced by $i=2$. Thus, the estimate (3.6) is proved.

It remains to prove the estimate (3.7)(iii). By the definition (3.5), we can write

$$
\begin{aligned}
\left\|\frac{d}{d x_{1}} M_{\varepsilon}^{1} u_{1}\right\|_{L^{2}\left(Q^{1}\right)} \leqq & \left\|m_{1} u_{1 x_{1}}\right\|_{L^{2}\left(Q^{1}\right)} \\
& +\frac{C}{x_{1 \varepsilon M}}\left(\left\|u_{1}-m_{1} u_{1}\right\|_{L^{2}\left(Q_{\varepsilon, M}^{1}\right)}+\left\|u_{1}-\bar{m}_{\varepsilon}^{1} u_{1}\right\|_{L^{2}\left(Q_{\varepsilon \varepsilon M}^{1}\right)}\right),
\end{aligned}
$$

which, by the estimates (3.9), (3.13) and (2.3'), implies that $\left\|\left(d / d x_{1}\right) M_{\varepsilon}^{1} u_{1}\right\|_{L^{2}\left(Q^{1}\right)} \leqq$ $C\|u\|_{V_{\varepsilon}}$. Since a similar estimate holds for $M_{\varepsilon}^{2} u_{2}$, we have proved the estimate (3.7)(iii).

REMARK 3.1. Actually, we can also define the operator $M_{\varepsilon}$ on the space $H_{\varepsilon}^{1}(Q)$. Of course, if $u$ belongs to $H_{\varepsilon}^{1}(Q) \backslash \mathscr{V}_{\varepsilon}$, then $M_{\varepsilon} u$ is not in $\mathscr{V}_{0}$. However, arguing as above, we can show that the estimates (3.6), (3.7) are also true for $u$ in $H_{\varepsilon}^{1}(Q)$. In particular, we can prove the following estimates, for $j=1,2$ :

$$
\begin{gathered}
\left\|u_{j}-M_{\varepsilon}^{j} u_{j}\right\|_{H^{1}\left(Q 2_{\varepsilon M}\right)} \leqq C \varepsilon\left[\left\|u_{j x_{j}}\right\|_{L^{2}\left(Q_{2 \varepsilon M}^{j}\right)}+\left\|\frac{1}{\varepsilon} u_{j x_{j+1}}\right\|_{L^{2}\left(Q_{2 \varepsilon M}^{j}\right)}\right], \\
\left\|u_{j}-M_{\varepsilon}^{j} u_{j}\right\|_{H^{1}\left(Q^{j}\right)} \leqq C \varepsilon\left[\left\|u_{j x_{j}}\right\|_{L^{2}\left(Q^{j}\right)}+\left\|\frac{1}{\varepsilon} u_{j x_{j+1}}\right\|_{L^{2}\left(Q^{j}\right)}\right],
\end{gathered}
$$

where $x_{3}$ denotes $x_{1}$.

In Sections 4-7, we often use the following estimates (see [13]):

$$
\begin{gathered}
\left\|m_{j} u_{j}\right\|_{H^{1}\left(Q^{j}\right)} \leqq\left\|u_{j}\right\|_{H^{1}\left(Q^{j}\right)}, \\
\left\|m_{j} u_{j}\right\|_{H^{1}\left(Q_{2 \varepsilon M}^{j}\right)} \leqq\left\|u_{j}\right\|_{H^{1}\left(Q_{2 \varepsilon M}^{j}\right)}, \\
\left\|u_{j}-m_{j} u_{j}\right\|_{H^{1}\left(Q_{2 \varepsilon M}^{j}\right)} \leqq C \varepsilon\left\|\frac{1}{\varepsilon} u_{j x_{j+1}}\right\|_{L^{2}\left(Q_{2 \varepsilon M}^{j}\right)} .
\end{gathered}
$$

REMARK 3.2. In Sections 8 and 9, we use the following property of the mapping $M_{\varepsilon} I_{\varepsilon}: \mathscr{V}_{0} \rightarrow \mathscr{V}_{0}$. For $0<\varepsilon \leqq \varepsilon_{0}$, for all $v \in \mathscr{V}_{0} \cap\left(H^{2}((0,1)) \times H^{2}((0,1))\right)$, we have

$$
\left\|v-M_{\varepsilon} I_{\varepsilon} v\right\|_{H^{1}((0,1)) \times H^{1}((0,1))} \leqq C \varepsilon^{\frac{1}{2}}\|v\|_{H^{2}((0,1)) \times H^{2}((0,1))}
$$


To prove this, we observe that, if $v=\left(v_{1}, v_{2}\right) \in \mathscr{V}_{0}$, then

$$
\begin{aligned}
& M_{\varepsilon}^{1} I_{\varepsilon}^{1} v_{1}-v_{1} \\
& \quad= \begin{cases}0 & x_{1} \in\left(2 x_{1 \varepsilon M}, 1\right], \\
\frac{x_{1}-x_{1 \varepsilon M}}{x_{1 \varepsilon M}} v_{1}\left(2\left(x_{1}-x_{1 \varepsilon M}\right)\right)-v_{1}\left(x_{1}\right)+\frac{2 x_{1 \varepsilon M}-x_{1}}{x_{1 \varepsilon M}} v_{1}(0) & x_{1} \in\left(x_{1 \varepsilon M}, 2 x_{1 \varepsilon M}\right], \\
v_{1}(0)-v_{1} & x_{1} \in\left[0, x_{1 \varepsilon M}\right]\end{cases}
\end{aligned}
$$

and

$$
\frac{d}{d x_{1}}\left(M_{\varepsilon}^{1} I_{\varepsilon}^{1} v_{1}-v_{1}\right)= \begin{cases}0 & x_{1} \in\left(2 x_{1 \varepsilon M}, 1\right] \\ D & x_{1} \in\left(x_{1 \varepsilon M}, 2 x_{1 \varepsilon M}\right] \\ -v_{1 x_{1}} & x_{1} \in\left[0, x_{1 \varepsilon M}\right]\end{cases}
$$

where

$$
D=\frac{1}{x_{1 \varepsilon M}} \int_{0}^{2\left(x_{1}-x_{1 \varepsilon M}\right)} v_{1 x_{1}}(s) d s+\frac{2\left(x_{1}-x_{1 \varepsilon M}\right)}{x_{1 \varepsilon M}} v_{1 x_{1}}\left(2\left(x_{1}-x_{1 \varepsilon M}\right)\right)-v_{1 x_{1}} .
$$

Using the Cauchy-Schwarz inequality, we obtain

$$
\left\|\frac{1}{x_{1 \varepsilon M}} \int_{0}^{2\left(x_{1}-x_{1 \varepsilon M}\right)} v_{1 x_{1}}(s) d s\right\|_{L^{2}\left(Q_{2 \varepsilon M}^{1}\right)} \leqq C \varepsilon^{\frac{1}{2}}\left\|v_{1}\right\|_{H^{1}((0,1))} .
$$

Likewise, using the Sobolev embedding of $H^{2}((0,1))$ into $L^{\infty}((0,1))$ and the CauchySchwarz inequality, we show that

$$
\begin{aligned}
\left\|v_{1 x_{1}}\right\|_{L^{2}\left(Q_{2 \varepsilon M}^{1}\right)} & +\left\|\frac{2\left(x_{1}-x_{1 \varepsilon M}\right)}{x_{1 \varepsilon M}} v_{1 x_{1}}\left(2\left(x_{1}-x_{1 \varepsilon M}\right)\right)\right\|_{L^{2}\left(Q_{2 \varepsilon M}^{1}\right)} \\
& \leqq C \varepsilon^{\frac{1}{2}}\left\|v_{1}\right\|_{H^{2}((0,1))} .
\end{aligned}
$$

From the equalities (3.18) and the estimates (3.19), (3.20), we deduce that

$$
\left\|\frac{d}{d x_{1}}\left(M_{\varepsilon}^{1} I_{\varepsilon}^{1} v_{1}-v_{1}\right)\right\|_{L^{2}((0.1))} \leqq C \varepsilon^{\frac{1}{2}}\left\|v_{1}\right\|_{H^{2}((0,1))} .
$$

A similar bound holds for $\left\|M_{\varepsilon}^{1} I_{\varepsilon}^{1} v_{1}-v_{1}\right\|_{L^{2}((0,1))}$. Since analogous estimates hold for $\left\|M_{\varepsilon}^{2} I_{\varepsilon}^{2} v_{2}-v_{2}\right\|_{H_{(}{ }_{(0,1))}},(3.16)$ is proved.

\section{An equation satisfied by the solution $v=\left(v_{1}, v_{2}\right)$ of the problem $(\tilde{\mathrm{P}})_{0}$}

We keep the notation of the previous sections; in particular, $M_{\varepsilon} u=\left(M_{\varepsilon}^{1} u_{1}, M_{\varepsilon}^{2} u_{2}\right)$ is defined by (3.5). If $v=\left(v_{1}, v_{2}\right)$ is any solution of $(\tilde{\mathrm{P}})_{0}$, then we have

$$
\left(v_{t}, M_{\varepsilon} u\right)_{H_{0}}+a_{0}\left(v, M_{\varepsilon} u\right)=-\left(\hat{f}(v)+\hat{G}_{0}, M_{\varepsilon} u\right)_{H_{0}} .
$$


By integration in $x_{1}, x_{2}$, we obtain

$$
\begin{aligned}
\sum_{j=1}^{2} \int_{Q^{j}} g_{j}\left[v_{j t} M_{\varepsilon}^{j} u_{j}\right. & \left.+v_{j x_{j}}\left(M_{\varepsilon}^{j} u_{j}\right)_{x_{j}}\right] d x_{1} d x_{2} \\
& =-\sum_{j=1}^{2} \int_{Q^{j}}\left(g_{j}\left[f\left(v_{j}\right)+G_{j 0}\right] M_{\varepsilon}^{j} u_{j}\right) d x_{1} d x_{2} .
\end{aligned}
$$

If we use the definition (3.5) of $M_{\varepsilon} u$ and perform some elementary calculations, we deduce that (4.2) is equivalent to the following equality:

$$
\begin{aligned}
\left(v_{t}, u\right)_{H_{\varepsilon}}+a_{\varepsilon}(v, u)= & -\left(\hat{f}(v)+\hat{G}_{0}, u\right)_{H_{\varepsilon}} \\
& +R_{\varepsilon}^{*}(v, u)+S_{\varepsilon}^{*}\left(\hat{f}(v)+\hat{G}_{0}, u\right)+L_{\varepsilon}^{*}\left(v_{t}, u\right),
\end{aligned}
$$

where

$$
\begin{aligned}
R_{\varepsilon}^{*}(v, u)= & -\sum_{j=1}^{2} \int_{Q \dot{z}_{\varepsilon M}^{j}} g_{j} v_{j x_{j}}\left(M_{\varepsilon}^{j} u_{j}-u_{j}\right)_{x_{j}} d x_{1} d x_{2} \\
& -\frac{1}{2} \sum_{j=1}^{2} \int_{J_{\varepsilon}^{j}} g_{j} v_{j x_{j}} u_{j x_{j}} d x_{1} d x_{2} \\
& -\sum_{j=1}^{2} \int_{Q^{j} \backslash J_{\varepsilon}^{j}} g_{j} v_{j x_{j}}\left(\frac{g_{j x_{j}}}{g_{j}}\right) x_{j+1} u_{j x_{j+1}} d x_{1} d x_{2} \\
& -\frac{1}{2} \sum_{j=1}^{2} \int_{J_{\varepsilon}^{j}} g_{j} v_{j x_{j}}\left(\frac{g_{j x_{j}}}{g_{j}}\right) x_{j+1} u_{j x_{j+1}} d x_{1} d x_{2},
\end{aligned}
$$

where we have let $x_{3}$ denote $x_{1}$,

$$
\begin{aligned}
& S_{\varepsilon}^{*}\left(\hat{f}(v)+\hat{G}_{0}, u\right)=\left(\hat{f}(v)+\hat{G}_{0}, u-M_{\varepsilon} u\right)_{H_{2 \varepsilon M}} \\
&-\frac{1}{2} \sum_{j=1}^{2} \int_{J_{\varepsilon}^{j}} g_{j}\left(f\left(v_{j}\right)+G_{j 0}\right) u_{j} d x_{1} d x_{2}, \\
& L_{\varepsilon}^{*}\left(v_{t}, u\right)=\left(v_{t}, u-M_{\varepsilon} u\right)_{H_{2 \varepsilon M}}-\frac{1}{2} \sum_{j=1}^{2} \int_{J_{\varepsilon}^{j}} g_{j} v_{j t} u_{j} d x_{1} d x_{2} .
\end{aligned}
$$

We also remark that, if $v=\left(v_{1}, v_{2}\right)$ is any solution of the problem $(\tilde{\mathrm{P}})_{0}$, we can write, for any $\xi=\left(\xi_{1}, \xi_{2}\right) \in \mathscr{V}_{0}$,

$$
\begin{aligned}
\left(v_{t}, \xi\right)_{H_{\varepsilon}}+a_{\varepsilon}(v, \xi)= & -\left(\hat{f}(v)+\hat{G}_{0}, \xi\right)_{H_{\varepsilon}} \\
& -\frac{1}{2} \sum_{j=1}^{2} \int_{J_{\varepsilon}^{j}} g_{j}\left[v_{j t} \xi_{j}+v_{j x_{j}} \xi_{j x_{j}}\right] d x_{1} d x_{2} \\
& -\frac{1}{2} \sum_{j=1}^{2} \int_{J_{\varepsilon}^{j}} g_{j}\left[f\left(v_{j}\right)+G_{j 0}\right] \xi_{j} d x_{1} d x_{2} .
\end{aligned}
$$

\section{An equation satisfied by the solution $\boldsymbol{u}^{\varepsilon}$ of problem $(\tilde{\mathbf{P}})_{\varepsilon}$}

If $u^{\varepsilon}=\left(u_{1}^{\varepsilon}, u_{2}^{\varepsilon}\right)$ is a solution of $(\tilde{\mathbf{P}})_{\varepsilon}$, we have, for any $\xi=\left(\xi_{1}, \xi_{2}\right) \in \mathscr{V}_{0}$,

$$
\left(u_{t}^{\varepsilon}, I_{\varepsilon} \xi\right)_{H_{\varepsilon}}+a_{\varepsilon}\left(u^{\varepsilon}, I_{\varepsilon} \xi\right)=-\left(\hat{f}\left(u^{\varepsilon}\right)+\hat{G}_{\varepsilon}, I_{\varepsilon} \xi\right)_{H_{\varepsilon}} \text {. }
$$


The definition of $I_{\varepsilon}$ and a few elementary computations yield

$$
\begin{aligned}
\left(u_{t}^{\varepsilon}, \xi\right)_{H_{\varepsilon}}+a_{\varepsilon}\left(u^{\varepsilon}, \xi\right)= & -\left(\hat{f}\left(u^{\varepsilon}\right)+\hat{G}_{\varepsilon}, \xi\right)_{H_{\varepsilon}} \\
& +R_{\varepsilon}\left(u^{\varepsilon}, \xi\right)+S_{\varepsilon}\left(\hat{f}\left(u^{\varepsilon}\right)+\hat{G}_{\varepsilon}, \xi\right)+L_{\varepsilon}\left(u_{t}^{\varepsilon}, \xi\right),
\end{aligned}
$$

where

$$
\begin{aligned}
R_{\varepsilon}\left(u^{\varepsilon}, \xi\right)= & -\sum_{j=1}^{2} \int_{Q \dot{z}_{\varepsilon M}} g_{j}\left(u_{j x_{j}}^{\varepsilon}-\frac{g_{j x_{j}}}{g_{j}} x_{j+1} u_{j x_{j}+1}^{\varepsilon}\right)\left(I_{\varepsilon} \xi-\xi\right)_{j x_{j}} d x_{1} d x_{2} \\
& -\frac{1}{2} \sum_{j=1}^{2} \int_{J_{\varepsilon}^{j}} g_{j}\left(u_{j x_{j}}^{\varepsilon}-\frac{g_{j x_{j}}}{g_{j}} x_{j+1} u_{j x_{j+1}}^{\varepsilon}\right) \xi_{j x_{j}} d x_{1} d x_{2} \\
S_{\varepsilon}\left(\hat{f}\left(u^{\varepsilon}\right)+\hat{G}_{\varepsilon}, \xi\right)= & \left(\hat{f}\left(u^{\varepsilon}\right)+\hat{G}_{\varepsilon}, \xi-I_{\varepsilon} \xi\right)_{H_{2 \varepsilon} M} \\
& -\frac{1}{2} \sum_{j=1}^{2} \int_{J_{\varepsilon}^{j}} g_{j}\left(f\left(u_{j}^{\varepsilon}\right)+G_{j \varepsilon}\right)\left(\xi_{j}-\xi_{j}(0)\right) d x_{1} d x_{2} \\
L_{\varepsilon}\left(u_{t}^{\varepsilon}, \xi\right)= & \left(u_{t}^{\varepsilon}, \xi-I_{\varepsilon} \xi\right)_{H_{2 \varepsilon M}}-\frac{1}{2} \sum_{j=1}^{2} \int_{J_{\varepsilon}^{j}} g_{j} u_{j t}^{\varepsilon}\left(\xi_{j}-\xi_{j}(0)\right) d x_{1} d x_{2} .
\end{aligned}
$$

We remark that the expression $a_{\varepsilon}\left(u^{\varepsilon}, \xi\right)$ has a rather simple form due to the fact that $\xi \in \mathscr{V}_{0}$. In fact,

$$
\begin{aligned}
a_{\varepsilon}\left(u^{\varepsilon}, \xi\right)= & \sum_{j=1}^{2} \int_{Q^{j} \backslash J_{\varepsilon}^{j}} g_{j}\left(u_{j x_{j}}^{\varepsilon}-\frac{g_{j x_{j}}}{g_{j}} x_{j+1} u_{j x_{j+1}}^{\varepsilon}\right) \xi_{j x_{j}} d x_{1} d x_{2} \\
& +\frac{1}{2} \sum_{j=1}^{2} \int_{J_{\varepsilon}^{j}} g_{j}\left(u_{j x_{j}}^{\varepsilon}-\frac{g_{j x_{j}}}{g_{j}} x_{j+1} u_{j x_{j+1}}^{\varepsilon}\right) \xi_{j x_{j}} d x_{1} d x_{2} .
\end{aligned}
$$

\section{Estimate of $\left\|T_{0}(t) M_{\varepsilon} u_{0}-T_{\varepsilon}(t) u_{0}\right\|_{L^{2}(Q)}$ for $u_{0} \in \mathscr{V}_{\varepsilon}$}

For given $u_{0} \in \mathscr{V}_{\varepsilon}$, we let $u^{\varepsilon}=T_{\varepsilon}(t) u_{0}, v=T_{0}(t) M_{\varepsilon} u_{0}$. Using the variational equalities $(\tilde{\mathrm{P}})_{\varepsilon}$ and $(\tilde{\mathrm{P}})_{0}$ satisfied by $u^{\varepsilon}$ and $v$, as well as the equalities (4.3), (4.4), (4.5), (5.2) and (5.3), we obtain

$$
\begin{aligned}
\left(u_{t}^{\varepsilon}-v_{t}, u^{\varepsilon}-v\right)_{H_{\varepsilon}}+a_{\varepsilon}\left(u^{\varepsilon}-v, u^{\varepsilon}-v\right)= & -\left(\hat{f}\left(u^{\varepsilon}\right)-\hat{f}(v), u^{\varepsilon}-v\right)_{H_{\varepsilon}}-\left(\hat{G}_{\varepsilon}-\hat{G}_{0}, u^{\varepsilon}-v\right)_{H_{\varepsilon}} \\
& +B_{\varepsilon}^{0}\left(u^{\varepsilon}, v\right)+B_{\varepsilon}^{1}\left(u^{\varepsilon}, v\right)+B_{\varepsilon}^{2}\left(u^{\varepsilon}, v\right),
\end{aligned}
$$

where

$$
\begin{aligned}
B_{\varepsilon}^{0}\left(u^{\varepsilon}, v\right)= & -\left(u_{t}^{\varepsilon}, v-I_{\varepsilon} v\right)_{H_{2 \varepsilon M}}-\left(v_{t}, u^{\varepsilon}-M_{\varepsilon} u^{\varepsilon}\right)_{H_{2 \varepsilon M}} \\
& +\frac{1}{2} \sum_{j=1}^{2} \int_{J_{\varepsilon}^{j}} g_{j} v_{j t}\left(u_{j}^{\varepsilon}-v_{j}\right) d x_{1} d x_{2} \\
& +\frac{1}{2} \sum_{j=1}^{2} \int_{J_{\varepsilon}^{j}} g_{j} u_{j t}^{\varepsilon}\left(v_{j}-v_{j}(0)\right) d x_{1} d x_{2}
\end{aligned}
$$




$$
\begin{aligned}
B_{\varepsilon}^{1}\left(u^{\varepsilon}, v\right)= & -\left(\hat{f}\left(u^{\varepsilon}\right)+\hat{G}_{\varepsilon}, v-I_{\varepsilon} v\right)_{H_{2 \varepsilon M}}-\left(\hat{f}(v)+\hat{G}_{0}, u^{\varepsilon}-M_{\varepsilon} u^{\varepsilon}\right)_{H_{2 \varepsilon M}} \\
& +\frac{1}{2} \sum_{j=1}^{2} \int_{J_{\varepsilon}^{j}} g_{j}\left(f\left(v_{j}\right)+G_{j 0}\right)\left(u_{j}^{\varepsilon}-v_{j}\right) d x_{1} d x_{2} \\
& +\frac{1}{2} \sum_{j=1}^{2} \int_{J_{\varepsilon}^{j}} g_{j}\left(f\left(u_{j}^{\varepsilon}\right)+G_{j \varepsilon}\right)\left(v_{j}-v_{j}(0)\right) d x_{1} d x_{2} ; \\
B_{\varepsilon}^{2}\left(u^{\varepsilon}, v\right)= & B_{\varepsilon}^{3}\left(u^{\varepsilon}, v\right)-\frac{1}{2} \sum_{j=1}^{2} \int_{J_{\varepsilon}^{j}} g_{j} v_{j x_{j}}^{2} d x_{1} d x_{2} \\
& +\sum_{j=1}^{2} \int_{Q z_{\varepsilon M}^{j} \backslash J_{\varepsilon}^{j}} g_{j} v_{j x_{j}}\left(M_{\varepsilon}^{j} u_{j}^{\varepsilon}-u_{j}^{\varepsilon}\right)_{x_{j}} d x_{1} d x_{2} \\
& +\sum_{j=1}^{2} \int_{Q_{2 \varepsilon M}^{j}} g_{j} u_{j x_{j}}^{\varepsilon}\left(I_{\varepsilon}^{j} v_{j}-v_{j}\right)_{x_{j}} d x_{1} d x_{2} ; \\
B_{\varepsilon}^{3}\left(u^{\varepsilon}, v\right)= & \sum_{j=1}^{2} \int_{Q^{j}} g_{j} v_{j x_{j}} \frac{g_{j x_{j}}}{g_{j}} x_{j+1} u_{j x_{j+1}}^{\varepsilon} d x_{1} d x_{2} \\
& +\sum_{j=1}^{2} \int_{Q \dot{z}_{\varepsilon M} \backslash J_{\varepsilon}^{j}} g_{j}\left(v_{j}-I_{\varepsilon}^{j} v_{j}\right)_{x_{j}} \frac{g_{j x_{j}}}{g_{j}} x_{j+1} u_{j x_{j+1}}^{\varepsilon} d x_{1} d x_{2} .
\end{aligned}
$$

In the estimation of the quantities in (6.2), we repeatedly use the following relation. For any $\xi_{j} \in H^{1}((0,1)), j=1,2$, we can write

$$
\left\|\xi_{j}\right\|_{L^{2}\left(Q_{2 \varepsilon M}^{\dot{z})}\right.} \leqq C \varepsilon^{\frac{1}{2}}\left\|\xi_{j}\right\|_{L^{\infty}((0,1))} \leqq C \varepsilon^{\frac{1}{2}}\left\|\xi_{j}\right\|_{H^{1}((0,1))} .
$$

Next we choose $0<\varepsilon_{0}<1$ and assume that $\left\|u_{0}\right\|_{r_{\varepsilon} \leqq} r_{0}$. We now estimate the expressions in (6.2), beginning with $B_{\varepsilon}^{0}\left(u^{\varepsilon}, v\right)$. Thanks to (3.3) and Lemma 2.3, we may write, for $0 \leqq \varepsilon \leqq \varepsilon_{0}$,

$$
\begin{aligned}
\left|\left(u_{t}^{\varepsilon}, v-I_{\varepsilon} v\right)_{H_{2 \varepsilon M}}\right| & +\frac{1}{2} \sum_{j=1}^{2}\left|\int_{J_{\varepsilon}^{j}} g_{j} u_{j t}^{\varepsilon}\left(v_{j}-v_{j}(0)\right) d x_{1} d x_{2}\right| \\
& \leqq C \varepsilon\left\|u_{t}^{\varepsilon}\right\|_{H_{2 \varepsilon M}}\|v\|_{H^{1}((0,1)) \times H^{1}((0,1))} \\
& \leqq C \varepsilon\left(\left\|u_{t}^{\varepsilon}\right\|_{H_{2 \varepsilon M}}^{2}+k\left(r_{0}\right)\right) .
\end{aligned}
$$

From (2.10) and (3.6), we deduce the inequality

$$
\left|\left(v_{t}, u^{\varepsilon}-M_{\varepsilon} u^{\varepsilon}\right)_{H_{2 \varepsilon M}}\right| \leqq C \varepsilon\left\|v_{t}\right\|_{H_{2 \varepsilon M}}\left\|u^{\varepsilon}\right\|_{V_{\varepsilon}} \leqq C \varepsilon\left[k\left(r_{0}\right)+\left\|v_{t}\right\|_{H_{2 \varepsilon M}}^{2}\right] .
$$

Using (2.10), (3.9) and (6.3), we can write, for any positive number $\delta$,

$$
\begin{aligned}
\sum_{j=1}^{2}\left|\int_{J_{\varepsilon}^{j}} g_{j} v_{j t}\left(u_{j}^{\varepsilon}-v_{j}\right) d x_{1} d x_{2}\right| \leqq & \sum_{j=1}^{2}\left|\int_{J_{\varepsilon}^{j}} g_{j} v_{j t}\left(m_{j} u_{j}^{\varepsilon}-v_{j}\right) d x_{1} d x_{2}\right| \\
& +\sum_{j=1}^{2}\left|\int_{J_{k}^{j}} g_{j} v_{j t}\left(m_{j} u_{j}^{\varepsilon}-u_{j}^{\varepsilon}\right) d x_{1} d x_{2}\right|
\end{aligned}
$$




$$
\begin{aligned}
& \leqq C\left\|v_{t}\right\|_{H_{2 \varepsilon} M}\left(\varepsilon\left\|u^{\varepsilon}\right\|_{V_{\varepsilon}}+\sum_{j=1}^{2} \varepsilon^{\frac{1}{2}}\left\|m_{j} u_{j}^{\varepsilon}-v_{j}\right\|_{H^{1}((0,1))}\right) \\
& \leqq \delta\left\|u^{\varepsilon}-v\right\|_{V_{\varepsilon}}^{2}+C \varepsilon\left\|u^{\varepsilon}\right\|_{V_{\varepsilon}}^{2}+C\left(1+\delta^{-1}\right) \varepsilon\left\|v_{t}\right\|_{H_{2 \varepsilon M}}^{2}
\end{aligned}
$$

From Lemma 2.3 and (6.2a), (6.4)-(6.6), we deduce that

$$
\left|B_{\varepsilon}^{0}\left(u^{\varepsilon}, v\right)\right| \leqq \delta\left\|u^{\varepsilon}-v\right\|_{V_{\varepsilon}}^{2}+C \varepsilon\left[k\left(r_{0}\right)+\left\|u_{t}^{\varepsilon}\right\|_{H_{2 \varepsilon}}^{2}+\left\|v_{t}\right\|_{H_{2 \varepsilon M}}^{2}\right]
$$

We now estimate $\left|B_{\varepsilon}^{1}\left(u^{\varepsilon}, v\right)\right|$. We first observe that the Mean Value Theorem implies that

$$
\left\|\hat{G}_{\varepsilon}-\hat{G}_{0}\right\|_{L^{2}(Q)} \leqq C \varepsilon\|G\|_{H^{1}(\tilde{Q})},
$$

and that the hypothesis (2.1) and the estimates (2.10) and (2.12) imply that

$$
\left\|f\left(u_{j}^{\varepsilon}\right)-f\left(v_{j}\right)\right\|_{L^{2}\left(Q^{j}\right)}^{2} \leqq C k_{1}\left(r_{0}\right)\left\|u_{j}^{\varepsilon}-v_{j}\right\|_{H^{1}\left(Q^{j}\right)}^{2},
$$

where $k_{1}\left(r_{0}\right)$ is a positive constant depending only on $r_{0}$.

Taking into account the estimates of Lemma 2.3 as well as the estimates (3.3), (3.6), we obtain

$$
\begin{aligned}
&\left|\left(\hat{f}\left(u^{\varepsilon}\right)+\hat{G}_{\varepsilon}, v-I_{\varepsilon} v\right)_{H_{2 \varepsilon} M}\right|+\left|\left(\hat{f}(v)+\hat{G}_{0}, u^{\varepsilon}-M_{\varepsilon} u^{\varepsilon}\right)_{H_{2 \varepsilon M}}\right| \\
& \quad+\frac{1}{2} \sum_{j=1}^{2}\left|\int_{J_{\varepsilon}^{j}} g_{j}\left(f\left(u_{j}^{\varepsilon}\right)+G_{j \varepsilon}\right)\left(v_{j}-I_{\varepsilon}^{j} v_{j}\right) d x_{1} d x_{2}\right| \\
& \leqq C \varepsilon k\left(r_{0}\right)\left(\left\|\hat{f}\left(u^{\varepsilon}\right)+\hat{G}_{\varepsilon}\right\|_{H_{2 \varepsilon M}}+\left\|\hat{f}(v)+\hat{G}_{0}\right\|_{H_{2 \varepsilon M}}\right) .
\end{aligned}
$$

Likewise, using, in addition, the estimates (3.9) and (6.3), we can write, for any positive number $\delta$,

$$
\begin{aligned}
& \frac{1}{2}\left|\sum_{j=1}^{2} \int_{J_{\varepsilon}^{j}} g_{j}\left(f\left(v_{j}\right)+G_{j 0}\right)\left(u_{j}^{\varepsilon}-v_{j}\right) d x_{1} d x_{2}\right| \\
& \leqq \sum_{j=1}^{2}\left|\int_{J_{\varepsilon}^{j}} g_{j}\left(f\left(v_{j}\right)+G_{j 0}\right)\left(m_{j} u_{j}^{\varepsilon}-u_{j}^{\varepsilon}\right) d x_{1} d x_{2}\right| \\
&+\sum_{j=1}^{2}\left|\int_{J_{\varepsilon}^{j}} g_{j}\left(f\left(v_{j}\right)+G_{j 0}\right)\left(m_{j} u_{j}^{\varepsilon}-v_{j}\right) d x_{1} d x_{2}\right| \\
& \leqq C\left\|\hat{f}(v)+\hat{G}_{0}\right\|_{H_{\varepsilon M}}\left(\varepsilon\left\|u^{\varepsilon}\right\|_{V_{\varepsilon}}+\sum_{j=1}^{2} \varepsilon^{\frac{1}{2}}\left\|m_{j} u_{j}^{\varepsilon}-v_{j}\right\|_{H^{1}((0,1))}\right) \\
& \leqq \delta\left\|u^{\varepsilon}-v\right\|_{V_{\varepsilon}}^{2}+C \varepsilon\left\|\hat{f}(v)+\hat{G}_{0}\right\|_{H_{\varepsilon M}}^{2}\left(\delta^{-1}+1\right)+C \varepsilon k\left(r_{0}\right) .
\end{aligned}
$$

From (6.2b), (6.10) and (6.11) as well as the hypotheses made on $f$ and $G$, we deduce at once that

$$
\left|B_{\varepsilon}^{1}\left(u^{\varepsilon}, v\right)\right| \leqq \delta\left\|u^{\varepsilon}-v\right\|_{H_{\varepsilon}^{1}(Q)}^{2}+\varepsilon C\left(1+\delta^{-1}\right) k\left(r_{0}\right) .
$$

We now turn to the estimate of $B_{\varepsilon}^{2}\left(u^{\varepsilon}, v\right)$. At first, we remark that, in the expression of $B_{\varepsilon}^{3}\left(u^{\varepsilon}, v\right)$, we can replace the expression $u_{j x_{j+1}}^{\varepsilon}$ by $\varepsilon \times \varepsilon^{-1}\left(u_{j}^{\varepsilon}-v_{j}\right)_{x_{j+1}}$. Taking into 
account this remark as well as the estimates (2.12) and (3.4), we can write

$$
\left|B_{\varepsilon}^{3}\left(u^{\varepsilon}, v\right)\right| \leqq C \varepsilon\|v\|_{H_{\varepsilon}^{1}(Q)}\left\|u^{\varepsilon}-v\right\|_{H_{\varepsilon}^{1}(Q)} \leqq C \varepsilon^{2} \delta^{-1} k\left(r_{0}\right)+\delta\left\|u^{\varepsilon}-v\right\|_{V_{\varepsilon}}^{2} .
$$

It remains to estimate the last two terms of $B_{\varepsilon}^{2}\left(u^{\varepsilon}, v\right)$. Using Lemma 2.3 and the estimates (3.7) (see also Remark 3.1) and (6.3), we have, for any positive number $\delta$,

$$
\begin{aligned}
& \sum_{j=1}^{2} \int_{Q_{L_{\varepsilon} M \backslash \backslash j}^{j}} g_{j} v_{j x_{j}}\left(M_{\varepsilon}^{j} u_{j}^{\varepsilon}-u_{j}^{\varepsilon}\right)_{x_{j}} d x_{1} d x_{2} \\
& =\sum_{j=1}^{2} \int_{Q \dot{L}_{\varepsilon M} \backslash J_{\varepsilon}^{j}} g_{j} v_{j x_{j}}\left(M_{\varepsilon}^{j}\left(u_{j}^{\varepsilon}-v_{j}\right)-\left(u_{j}^{\varepsilon}-v_{j}\right)\right)_{x_{j}} d x_{1} d x_{2} \\
& +\sum_{j=1}^{2} \int_{Q \dot{z}_{c M} \backslash \backslash_{\varepsilon}^{j}} g_{j} v_{j x_{j}}\left(M_{\varepsilon}^{j} v_{j}-v_{j}\right)_{x_{j}} d x_{1} d x_{2} \\
& \leqq C \varepsilon^{\frac{1}{2}}\|v\|_{H^{2}((0,1)) \times H^{2}((0,1))}\left(\left\|u^{\varepsilon}-v\right\|_{V_{\varepsilon}}+C\|v\|_{H^{1}\left(\left(0,2 x_{1 e M}\right)\right) \times H^{1}\left(\left(0,2 x_{2 e M}\right)\right)}\right) \\
& \leqq \delta\left\|u^{\varepsilon}-v\right\|_{V_{\varepsilon}}^{2}+C \varepsilon\left(1+\delta^{-1}\right)\|v\|_{H^{2}}^{2}((0,1)) \times H^{2}((0,1)) .
\end{aligned}
$$

We write the last term in $(6.2 \mathrm{c})$ in the following way:

$$
\begin{aligned}
& \sum_{j=1}^{2} \int_{Q Q_{\varepsilon M}^{j}} g_{j} u_{j x_{j}}^{\varepsilon}\left(I_{\varepsilon}^{j} v_{j}-v_{j}\right)_{x_{j}} d x_{1} d x_{2} \\
&=\sum_{j=1}^{2} \int_{Q \dot{z}_{\varepsilon M}} g_{j}\left(u_{j x_{j}}^{\varepsilon}-v_{j x_{j}}\right)\left(I_{\varepsilon}^{j} v_{j}-v_{j}\right)_{x_{j}} d x_{1} d x_{2} \\
&+\sum_{j=1}^{2} \int_{Q \dot{Q}_{\varepsilon M} \backslash Q_{\varepsilon M}^{j}} 2 g_{j} v_{j x_{j}}\left(v_{j x_{j}}\left(2\left(x_{j}-x_{j \varepsilon M}\right)\right)-v_{j x_{j}}\left(x_{j}\right)\right) d x_{1} d x_{2} \\
&+\sum_{j=1}^{2} \int_{Q_{i M}^{j}}\left(g_{j}\left(x_{j}+x_{j \varepsilon M}\right)-g_{j}\left(x_{j}\right)\right)\left(v_{j x_{j}}\left(x_{j}+x_{j \varepsilon M}\right)\right)^{2} d x_{1} d x_{2} \\
&+\sum_{j=1}^{2} \int_{Q_{\varepsilon}^{j} M} g_{j}\left(\left(v_{j x_{j}}\left(x_{j}+x_{j \varepsilon M}\right)\right)^{2}-\left(v_{j x_{j}}\left(x_{j}\right)\right)^{2}\right) d x_{1} d x_{2},
\end{aligned}
$$

which, thanks to Lemma 2.3, and the estimates (3.3) and (6.3), implies that, for any positive number $\delta$,

$$
\begin{aligned}
\left|\sum_{j=1}^{2} \int_{Q z_{\varepsilon M}^{j}} g_{j} u_{j x_{j}}^{\varepsilon}\left(I_{\varepsilon}^{j} v_{j}-v_{j}\right)_{x_{j}} d x_{1} d x_{2}\right| \leqq & C \delta^{-1} \varepsilon\|v\|_{H^{2}((0,1)) \times H^{2}((0,1))}^{2}+\delta\left\|u^{\varepsilon}-v\right\|_{V_{\varepsilon}}^{2} \\
& +C \varepsilon^{3 / 2}\|v\|_{H^{2}((0,1)) \times H^{2}((0,1))}^{2} \\
& +C \varepsilon^{2} \sum_{j=1}^{2}\left\|g_{j x_{j}}\right\|_{L^{\infty}((0,1))}\left\|v_{j}\right\|_{H^{2}((0,1))} .
\end{aligned}
$$

From (6.2c), (6.2d), (6.13)-(6.16) as well as from Lemma 2.3, we obtain

$$
\begin{aligned}
\left|B_{\varepsilon}^{2}\left(u^{\varepsilon}, v\right)\right| \leqq & \delta\left\|u^{\varepsilon}-v\right\|_{V_{\varepsilon}}^{2}+C \varepsilon\left(1+\delta^{-1}\right)\|v\|_{H^{2}((0,1)) \times H^{2}((0,1))}^{2} \\
& +C \varepsilon\left(1+\delta^{-1}\right) k\left(r_{0}\right) .
\end{aligned}
$$


Finally, from the equalities (6.1), (6.2) and the estimates (6.7) $-(6.9),(6.12),(6.17)$, we deduce that, for any positive constant $\delta$,

$$
\begin{aligned}
\frac{1}{2} \frac{\partial}{\partial t}\left\|u_{t}^{\varepsilon}-v_{t}\right\|_{H_{\varepsilon}}^{2}+ & a_{\varepsilon}\left(u^{\varepsilon}-v, u^{\varepsilon}-v\right) \\
\leqq & \delta\left\|u^{\varepsilon}-v\right\|_{V_{\varepsilon}}^{2}+C\left(1+\delta^{-1} k_{1}\left(r_{0}\right)\right)\left\|u^{\varepsilon}-v\right\|_{H_{\varepsilon}}^{2} \\
& +C \varepsilon\left(1+\delta^{-1}\right)\left(k\left(r_{0}\right)+1\right) \\
& +C \varepsilon\left(1+\delta^{-1}\right)\|v\|_{H^{2}((0,1)) \times H^{2}((0,1))}^{2} \\
& +C \varepsilon\left(\left\|u_{t}\right\|_{H_{\varepsilon}}^{2}+\left\|v_{t}\right\|_{H_{\varepsilon}}^{2}\right) .
\end{aligned}
$$

If we choose $\delta$ sufficiently small and integrate the inequality (6.18) from 0 to $t$, we obtain that, for $t \geqq 0$,

$$
\begin{aligned}
\left\|u^{\varepsilon}(t)-v(t)\right\|_{H_{\varepsilon}}^{2} & +\int_{0}^{t}\left\|u^{\varepsilon}-v\right\|_{V_{\varepsilon}}^{2} d s \leqq C\left\|u_{0}-M_{\varepsilon} u_{0}\right\|_{H_{\varepsilon}}^{2} \\
& +C \varepsilon k\left(r_{0}\right) t+C\left(1+k_{1}\left(r_{0}\right)\right) \int_{0}^{t}\left\|u^{\varepsilon}-v\right\|_{H_{\varepsilon}}^{2} d s \\
& +C \varepsilon \int_{0}^{t}\left(\left\|u_{t}^{\varepsilon}\right\|_{H_{\varepsilon}}^{2}+\left\|v_{t}\right\|_{H_{\varepsilon}}^{2}+\|v\|_{H^{2}((0,1)) \times H^{2}((0,1))}^{2}\right) d s .
\end{aligned}
$$

Finally, applying the Gronwall lemma to (6.19) and using Lemma 2.3 and the estimate (3.6), we conclude that, for $t \geqq 0$, for $u_{0} \in \mathscr{V}_{\varepsilon},\left\|u_{0}\right\|_{\mathscr{\gamma}_{\varepsilon}} \leqq r_{0}$,

$\left\|T_{\varepsilon}(t) u_{0}-T_{0}(t) M_{\varepsilon} u_{0}\right\|_{L^{2}(Q)}^{2}+\int_{0}^{t}\left\|T_{\varepsilon}(s) u_{0}-T_{0}(s) M_{\varepsilon} u_{0}\right\|_{V_{\varepsilon}}^{2} d s \leqq C \varepsilon k\left(r_{0}\right) e^{k\left(r_{0}\right) t}$

\section{Estimate of $\left\|t T_{0}(t) M_{\varepsilon} u_{0}-t T_{\varepsilon}(t) u_{0}\right\|_{H_{\varepsilon}(Q)}$ for $u_{0} \in \mathscr{V}_{\varepsilon}$}

As in the previous section, for given $u_{0} \in \mathscr{V}_{\varepsilon}$, we let $u^{\varepsilon}=T_{\varepsilon}(t) u_{0}, v=T_{0}(t) M_{\varepsilon} u_{0}$ and we assume that $\left\|u_{0}\right\|_{r_{\varepsilon}} \leqq r_{0}$. We also use the notation $z^{\varepsilon}=t u^{\varepsilon}, z^{0}=t v$. Since $z^{\varepsilon}$ and $z^{0}$ belong to $\mathscr{V}_{\varepsilon}$ and $\mathscr{V}_{0}$, respectively, we can use the variational equalities $(\tilde{\mathrm{P}})_{\varepsilon}$ and $(\tilde{\mathbf{P}})_{0}$ satisfied by $u^{\varepsilon}$ and $v$, as well as the equalities (4.3)-(4.5), (5.2) and (5.3), to obtain

$$
\begin{aligned}
\left\|\left(z^{\varepsilon}-z^{0}\right)_{t}\right\|_{H_{\varepsilon}}^{2}+ & \frac{1}{2} \frac{d}{d t} a_{\varepsilon}\left(z^{\varepsilon}-z^{0}, z^{\varepsilon}-z^{0}\right) \\
= & -t\left(\hat{f}\left(u^{\varepsilon}\right)-\hat{f}(v), z_{t}^{\varepsilon}-z_{t}^{0}\right)_{H_{\varepsilon}}-t\left(\hat{G}_{\varepsilon}-\hat{G}_{0}, z_{t}^{\varepsilon}-z_{t}^{0}\right)_{H_{\varepsilon}} \\
& +\left(u^{\varepsilon}-v, z_{t}^{\varepsilon}-z_{t}^{0}\right)_{H_{\varepsilon}}+\tilde{B}_{\varepsilon}^{0}\left(u^{\varepsilon}, v\right)+\tilde{B}_{\varepsilon}^{1}\left(u^{\varepsilon}, v\right)+\tilde{B}_{\varepsilon}^{2}\left(u^{\varepsilon}, v\right),
\end{aligned}
$$


where

$$
\begin{aligned}
& \tilde{B}_{\varepsilon}^{0}\left(u^{\varepsilon}, v\right)=-\left(t u_{t}^{\varepsilon}, z_{t}^{0}-I_{\varepsilon} z_{t}^{0}\right)_{H_{2 \varepsilon M}}-\left(t v_{t}, z_{t}^{\varepsilon}-M_{\varepsilon} z_{t}^{\varepsilon}\right)_{H_{2 \varepsilon M}} \\
& +\frac{1}{2} \sum_{j=1}^{2} \int_{J_{\varepsilon}^{j}} \operatorname{tg}_{j} v_{j t}\left(z_{j t}^{\varepsilon}-z_{j t}^{0}\right) d x_{1} d x_{2} \\
& +\frac{1}{2} \sum_{j=1}^{2} \int_{J_{\varepsilon}^{j}} \operatorname{tg}_{j} u_{j t}^{\varepsilon}\left(z_{j t}^{0}-z_{j t}^{0}(0)\right) d x_{1} d x_{2} ; \\
& \tilde{B}_{\varepsilon}^{1}\left(u^{\varepsilon}, v\right)=-t\left(\hat{f}\left(u^{\varepsilon}\right)+\hat{G}_{\varepsilon}, z_{t}^{0}-I_{\varepsilon} z_{t}^{0}\right)_{H_{2 \varepsilon M}}-t\left(\hat{f}(v)+\hat{G}_{0}, z_{t}^{\varepsilon}-M_{\varepsilon} z_{t}^{\varepsilon}\right)_{H_{2 \varepsilon M}} \\
& +\frac{1}{2} \sum_{j=1}^{2} \int_{J_{\varepsilon}^{j}} t g_{j}\left(f\left(v_{j}\right)+G_{j 0}\right)\left(z_{j t}^{\varepsilon}-z_{j t}^{0}\right) d x_{1} d x_{2} \\
& +\frac{1}{2} \sum_{j=1}^{2} \int_{J_{\varepsilon}^{j}} \operatorname{tg}_{j}\left(f\left(u_{j}^{\varepsilon}\right)+G_{j \varepsilon}\right)\left(z_{j t}^{0}-z_{j t}^{0}(0)\right) d x_{1} d x_{2} \\
& \tilde{B}_{\varepsilon}^{2}\left(u^{\varepsilon}, v\right)=\tilde{B}_{\varepsilon}^{3}\left(u^{\varepsilon}, v\right)+\sum_{j=1}^{2} \int_{Q \dot{z}_{\varepsilon} M} t g_{j} v_{j x_{j}}\left(M_{\varepsilon}^{j} z_{j t}^{\varepsilon}-z_{j t}^{\varepsilon}\right)_{x_{j}} d x_{1} d x_{2} \\
& +\sum_{j=1}^{2} \int_{Q \dot{2}_{\mathrm{E} M}} \operatorname{tg}_{j} u_{j x_{j}}^{\varepsilon}\left(I_{\varepsilon}^{j} z_{j t}^{0}-z_{j t}^{0}\right)_{x_{j}} d x_{1} d x_{2} \\
& +\frac{1}{2} \sum_{j=1}^{2} \int_{J_{\varepsilon}^{j}} \operatorname{tg}_{j}\left[v_{j x_{j}}\left(z_{j t x_{j}}^{\varepsilon}-z_{j t x_{j}}^{0}\right)+u_{j x_{j}}^{\varepsilon} z_{j t x_{j}}^{0}\right] d x_{1} d x_{2} ; \\
& \tilde{B}_{\varepsilon}^{3}\left(u^{\varepsilon}, v\right)=\sum_{j=1}^{2} \int_{Q \dot{q}_{\varepsilon M}} \operatorname{tg} \frac{g_{j x_{j}}}{g_{j}} x_{j+1} u_{j x_{j+1}}^{\varepsilon}\left(z_{j t}^{0}-I_{\varepsilon}^{j} z_{j t}^{0}\right)_{x_{j}} d x_{1} d x_{2} \\
& +\sum_{j=1}^{2} \int_{Q^{j}} \operatorname{tg}_{j} v_{j x_{j}} \frac{g_{j x_{j}}}{g_{j}} x_{j+1} z_{j t x_{j+1}}^{\varepsilon} d x_{1} d x_{2} \\
& -\frac{1}{2} \sum_{j=1}^{2} \int_{J_{\varepsilon}^{j}} \operatorname{tg} \frac{g_{j x_{j}}}{g_{j}} x_{j+1}\left(u_{j x_{j+1}}^{\varepsilon} z_{j t x_{j}}^{0}+v_{j x_{j}} z_{j t x_{j+1}}^{\varepsilon}\right) d x_{1} d x_{2} .
\end{aligned}
$$

Our next objective is to estimate the terms $\tilde{B}_{\varepsilon}^{0}\left(u^{\varepsilon}, v\right), \tilde{B}_{\varepsilon}^{1}\left(u^{\varepsilon}, v\right), \tilde{B}_{\varepsilon}^{2}\left(u^{\varepsilon}, v\right)$ and $\tilde{B}_{\varepsilon}^{3}\left(u^{\varepsilon}, v\right)$. Thanks to Lemma 2.3 and to the estimates (3.3) and (3.6), we may write, for $0 \leqq \varepsilon \leqq \varepsilon_{0}$,

$$
\begin{aligned}
\left|\left(t u_{t}^{\varepsilon}, z_{t}^{0}-I_{\varepsilon} z_{t}^{0}\right)_{H_{2 \varepsilon M}}\right|+\frac{1}{2} \mid & \sum_{j=1}^{2} \int_{J_{\varepsilon}^{j}} t g_{j} u_{j t}^{\varepsilon}\left(z_{j t}^{0}-z_{j t}^{0}(0)\right) d x_{1} d x_{2} \mid \\
& \leqq C \varepsilon\left\|t u_{t}^{\varepsilon}\right\|_{H_{\varepsilon}}\left\|z_{t}^{0}\right\|_{H^{1}((0,1)) \times H^{1}((0,1))} \\
& \leqq C \varepsilon k\left(r_{0}\right)\left(1+t^{2}\right),
\end{aligned}
$$

and

$$
\left|\left(t v_{t}, z_{t}^{\varepsilon}-M_{\varepsilon} z_{t}^{\varepsilon}\right)_{H_{2 \varepsilon M}}\right| \leqq C \varepsilon\left\|t v_{t}\right\|_{H_{2 \varepsilon M}}\left\|z_{t}^{\varepsilon}\right\|_{\mathscr{r}_{\varepsilon}} \leqq C \varepsilon k\left(r_{0}\right)\left(1+t^{2}\right)
$$

Likewise, using Lemma 2.3 and estimate (6.3), we obtain, for any positive 
number $\delta$,

$$
\begin{aligned}
\frac{1}{2}\left|\sum_{j=1}^{2} \int_{J_{\varepsilon}^{j}} t g_{j} v_{j t}\left(z_{j t}^{\varepsilon}-z_{j t}^{0}\right) d x_{1} d x_{2}\right| & \leqq C\left\|z_{t}^{\varepsilon}-z_{t}^{0}\right\|_{H_{2 \varepsilon M}}\left\|t v_{t}\right\|_{H_{2 \varepsilon M}} \\
& \leqq \delta\left\|z_{t}^{\varepsilon}-z_{t}^{0}\right\|_{H_{\varepsilon}}^{2}+C \varepsilon \delta^{-1} k\left(r_{0}\right)\left(1+t^{2}\right) .
\end{aligned}
$$

From the estimates (7.3)-(7.5), we deduce that

$$
\left|\tilde{B}_{\varepsilon}^{0}\left(u^{\varepsilon}, v\right)\right| \leqq \delta\left\|z_{t}^{\varepsilon}-z_{t}^{0}\right\|_{H_{\varepsilon}}^{2}+C \varepsilon\left(1+\delta^{-1}\right) k\left(r_{0}\right)\left(1+t^{2}\right) .
$$

We now turn to the estimate of $\tilde{B}_{\varepsilon}^{1}\left(u^{\varepsilon}, v\right)$. As in Section 6 , taking into account Lemma 2.3 and the estimates (3.3) and (3.6), we write

$$
\begin{gathered}
\left|t\left(\hat{f}\left(u^{\varepsilon}\right)+\hat{G}_{\varepsilon}, z_{t}^{0}-I_{\varepsilon} z_{t}^{0}\right)_{H_{2 \varepsilon M}}\right|+\left|t\left(\hat{f}(v)+\hat{G}_{0}, z_{t}^{\varepsilon}-M_{\varepsilon} z_{t}^{\varepsilon}\right)_{H_{2 \varepsilon M}}\right| \\
\quad+\frac{1}{2}\left|\sum_{j=1}^{2} \int_{J_{\varepsilon}^{j}} \operatorname{tg}\left(f\left(u_{j}^{\varepsilon}\right)+G_{j \varepsilon}\right)\left(z_{j t}^{0}-z_{j t}^{0}(0)\right) d x_{1} d x_{2}\right| \\
\leqq C \varepsilon\left(1+t^{2}\right)\left(1+k\left(r_{0}\right)\right) .
\end{gathered}
$$

Since we do not want to use a strong hypothesis on $G$, the estimate of the next term in $\tilde{B}_{\varepsilon}^{1}\left(u^{\varepsilon}, v\right)$ is a little more delicate. We can write

$$
\begin{aligned}
& \frac{1}{2} \sum_{j=1}^{2} \int_{J_{\varepsilon}^{j}} \operatorname{tg}_{j}\left(f\left(v_{j}\right)+G_{j 0}\right)\left(z_{j t}^{\varepsilon}-z_{j t}^{0}\right) d x_{1} d x_{2} \\
& =\frac{d}{d t} \tilde{B}_{\varepsilon}^{4}(t)-\frac{1}{2} \sum_{j=1}^{2} \int_{J_{\varepsilon}^{j}} g_{j}\left(f\left(v_{j}\right)+G_{j 0}+f^{\prime}\left(v_{j}\right) v_{j t}\right)\left(z_{j}^{\varepsilon}-z_{j}^{0}\right) d x_{1} d x_{2},
\end{aligned}
$$

where

$$
\tilde{B}_{\varepsilon}^{4}(t)=\frac{1}{2} \sum_{j=1}^{2} \int_{J_{\varepsilon}^{j}} t_{j}\left(f\left(v_{j}\right)+G_{j 0}\right)\left(z_{j}^{\varepsilon}-z_{j}^{0}\right) d x_{1} d x_{2} .
$$

But, using the hypothesis (2.2), the estimates (3.9), (6.3) and Lemma 2.3, we obtain

$$
\begin{aligned}
\frac{1}{2} \mid \sum_{j=1}^{2} & \int_{J_{\varepsilon}^{j}} g_{j}\left(f\left(v_{j}\right)+G_{j 0}+f^{\prime}\left(v_{j}\right) v_{j t}\right)\left(z_{j}^{\varepsilon}-z_{j}^{0}\right) d x_{1} d x_{2} \mid \\
\leqq & C \sum_{j=1}^{2} t\left\|f\left(v_{j}\right)+G_{j 0}+f^{\prime}\left(v_{j}\right) v_{j t}\right\|_{L^{2}\left(J_{\varepsilon}^{j}\right)}\left(\left\|m_{j} u_{j}^{\varepsilon}-u_{j}^{\varepsilon}\right\|_{L^{2}\left(J_{\varepsilon}^{j}\right)}+\left\|u_{j}^{\varepsilon}-v_{j}\right\|_{L^{2}\left(J_{\xi}^{j}\right)}\right) \\
\leqq & C\left[\left\|t v_{t}\right\|_{H^{1}((0,1)) \times H^{1}((0,1))}\left(1+\|v\|_{H^{1}((0,1)) \times H^{1}((0,1))}^{1_{1}^{1}}\right)+t\left(1+k\left(r_{0}\right)\right)\right] \\
& \times\left(\varepsilon\left\|u^{\varepsilon}\right\|_{\mathscr{r}_{\varepsilon}}+\varepsilon^{\frac{1}{2}}\left\|u^{\varepsilon}-v\right\|_{H_{\varepsilon}^{1}(Q)}\right) \\
\leqq & C\left(\varepsilon\left(1+t^{2}\right)\left(1+k\left(r_{0}\right)\right)+\left\|u^{\varepsilon}-v\right\|_{H_{\varepsilon}^{1}(Q)}^{2}\right) .
\end{aligned}
$$

From the equalities (7.2b) and (7.8) and the estimates (7.7), (7.9), we at once derive that

$$
\begin{aligned}
\left|\tilde{B}_{\varepsilon}^{1}\left(u^{\varepsilon}, v\right)\right| \leqq & \frac{1}{2} \sum_{j=1}^{2} \frac{d}{d t}\left(\int_{J_{\varepsilon}^{j}} \operatorname{tg}_{j}\left(f\left(v_{j}\right)+G_{j 0}\right)\left(z_{j}^{\varepsilon}-z_{j}^{0}\right) d x_{1} d x_{2}\right) \\
& +\left\|u^{\varepsilon}-v\right\|_{H_{\varepsilon}^{1}(Q)}^{2}+C \varepsilon\left(1+t^{2}\right)\left(1+k\left(r_{0}\right)\right) .
\end{aligned}
$$


It remains to estimate $\tilde{B}_{\varepsilon}^{3}\left(u^{\varepsilon}, v\right)$ and $\tilde{B}_{\varepsilon}^{2}\left(u^{\varepsilon}, v\right)$. Arguing as for the estimate of $\left|B_{\varepsilon}^{3}\left(u^{\varepsilon}, v\right)\right|$ in Section 6 and using the estimates (3.4) and Lemma 2.3, we may write

$$
\begin{aligned}
\left|\tilde{B}_{\varepsilon}^{3}\left(u^{\varepsilon}, v\right)\right| & \leqq C \varepsilon t\left(\left\|u^{\varepsilon}\right\|_{\mathscr{V}_{\varepsilon}}\left\|z_{t}^{0}\right\|_{H^{1}((0,1)) \times H^{1}((0,1))}+\left\|z_{t}^{\varepsilon}\right\|_{\mathscr{V}_{\varepsilon}}\|v\|_{H^{1}((0,1)) \times H^{1}((0,1))}\right) \\
& \leqq C \varepsilon\left(1+t^{2}\right) k\left(r_{0}\right) .
\end{aligned}
$$

Arguing as in the proof of the estimate (6.14) by using Lemma 2.3 and the estimates (3.7) and (6.3), we obtain

$$
\begin{aligned}
& \sum_{j=1}^{2} \int_{Q \dot{Z}_{\varepsilon M}} t g_{j} v_{j x_{j}}\left(M_{\varepsilon}^{j} z_{j t}^{\varepsilon}-z_{j t}^{\varepsilon}\right)_{x_{j}} d x_{1} d x_{2} \\
&=\frac{d}{d t} \tilde{B}_{\varepsilon}^{5}(t)-\sum_{j=1}^{2} \int_{Q \dot{z}_{\varepsilon M}} g_{j}\left(t v_{j t x_{j}}+v_{j x_{j}}\right)\left(M_{\varepsilon}^{j} z_{j}^{\varepsilon}-z_{j}^{\varepsilon}\right)_{x_{j}} d x_{1} d x_{2} \\
& \leqq \frac{d}{d t} \tilde{B}_{\varepsilon}^{5}(t)+C \varepsilon^{\frac{1}{2}}\left\|z_{t}^{0}\right\|_{H^{2}((0,1)) \times H^{2}((0,1))} \\
& \times\left[t\left\|u^{\varepsilon}-v\right\|_{H_{\varepsilon}^{1}(Q)}+t \sum_{j=1}^{2}\left\|v_{j}\right\|_{H^{1}\left(Q \dot{z}_{\varepsilon}\right)}\right] \\
& \leqq \frac{d}{d t} \tilde{B}_{\varepsilon}^{5}(t)+t^{2}\left\|u^{\varepsilon}-v\right\|_{H_{\varepsilon}^{1}(Q)}^{2} \\
&+C \varepsilon\left(\left\|z_{t}^{0}\right\|_{H^{2}((0,1)) \times H^{2}((0,1))}^{2}+t^{2}\|v\|_{H^{2}((0,1)) \times H^{2}((0,1))}^{2}\right)
\end{aligned}
$$

where

$$
\tilde{B}_{\varepsilon}^{5}(t)=\sum_{j=1}^{2} \int_{Q \dot{z}_{\varepsilon M}} \operatorname{tg}_{j} v_{j x_{j}}\left(M_{\varepsilon}^{j} z_{j}^{\varepsilon}-z_{j}^{\varepsilon}\right)_{x_{j}} d x_{1} d x_{2} .
$$

Using Lemma 2.3 and the estimates (3.4) and (6.3), we are able to estimate the next term in $\tilde{B}_{\varepsilon}^{2}\left(u^{\varepsilon}, v\right)-\tilde{B}_{\varepsilon}^{3}\left(u^{\varepsilon}, v\right)$ as follows:

$$
\begin{aligned}
&\left|\sum_{j=1}^{2} \int_{Q \dot{z}_{\varepsilon M}} \operatorname{tg}_{j} u_{j x_{j}}^{\varepsilon}\left(I_{\varepsilon}^{j} z_{j t}^{0}-z_{j t}^{0}\right)_{x_{j}} d x_{1} d x_{2}\right| \\
&=\mid \sum_{j=1}^{2} \int_{Q_{2 \varepsilon M}^{j}} \operatorname{tg}_{j}\left(u_{j x_{j}}^{\varepsilon}-v_{j x_{j}}\right)\left(I_{\varepsilon}^{j} z_{j t}^{0}-z_{j t}^{0}\right)_{x_{j}} d x_{1} d x_{2} \\
&+\sum_{j=1}^{2} \int_{Q \dot{z}_{\varepsilon M}} \operatorname{tg}_{j} v_{j x_{j}}\left(I_{\varepsilon}^{j} z_{j t}^{0}-z_{j t}^{0}\right)_{x_{j}} d x_{1} d x_{2} \mid \\
& \leqq C \sum_{j=1}^{2}\left(t\left\|u_{j x_{j}}^{\varepsilon}-v_{j x_{j}}\right\|_{L^{2}\left(Q \dot{z}_{\varepsilon M}\right)}\left\|z_{j t}^{0}\right\|_{H^{1}\left(Q \dot{q}_{\varepsilon M}\right)}\right. \\
&\left.+t\left\|v_{j x_{j}}\right\|_{L^{2}\left(Q \dot{z}_{\varepsilon M}\right)}\left\|z_{j t}^{0}\right\|_{H^{1}\left(Q \dot{z}_{\varepsilon M}\right)}\right) \\
& \leqq t^{2}\left\|u^{\varepsilon}-v\right\|_{H_{\varepsilon}^{1}(Q)}^{2}+C \varepsilon t^{2}\|v\|_{H^{2}((0,1)) \times H^{2}((0,1))}^{2} \\
&+C \varepsilon\left\|z_{t}^{0}\right\|_{H^{2}((0,1)) \times H^{2}((0,1))}^{2} \\
& \leqq t^{2}\left\|u^{\varepsilon}-v\right\|_{H_{\varepsilon}^{1}(Q)}^{2}+C \varepsilon z_{t}^{0} \|_{H^{2}((0,1)) \times H^{2}((0,1))}^{2}+C \varepsilon k\left(r_{0}\right)\left(1+t^{2}\right) .
\end{aligned}
$$


Finally, we have:

$$
\begin{aligned}
\frac{1}{2} \sum_{j=1}^{2} \int_{J_{\varepsilon}^{j}} \operatorname{tg}_{j}\left[v_{j x_{j}}\left(z_{j t x_{j}}^{\varepsilon}-z_{j t x_{j}}^{0}\right)+u_{j x_{j}}^{\varepsilon} z_{j t x_{j}}^{0}\right] d x_{1} d x_{2} \\
\equiv \frac{d}{d t} \tilde{B}_{\varepsilon}^{6}(t) \equiv \frac{1}{2} \sum_{j=1}^{2} \frac{d}{d t} \int_{J_{\varepsilon}^{j}} g_{j} z_{j x_{j}}^{0}\left(z_{j x_{j}}^{\varepsilon}-z_{j x_{j}}^{0}\right) d x_{1} d x_{2} \\
+\frac{1}{4} \sum_{j=1}^{2} \frac{d}{d t} \int_{J_{\varepsilon}^{j}} g_{j}\left(z_{j x_{j}}^{0}\right)^{2} d x_{1} d x_{2} .
\end{aligned}
$$

Integrating the equality (7.1) from 0 to $t$ and using the equalities (7.2) as well as the estimates (6.8), (6.9), (7.6), (7.10)-(7.12), (7.14), (7.15) and Lemma 2.3, we obtain, for $t \geqq 0$,

$$
\begin{aligned}
& \int_{0}^{t}\left\|z_{t}^{\varepsilon}(s)-z_{t}^{0}(s)\right\|_{H_{\varepsilon}}^{2} d s+a_{\varepsilon}\left(z^{\varepsilon}(t)-z^{0}(t), z^{\varepsilon}(t)-z^{0}(t)\right) \\
& \leqq C \varepsilon\left(1+t^{2}+t^{3}\right)\left(1+k\left(r_{0}\right)\right)+C \int_{0}^{t}\left\|u^{\varepsilon}(s)-v(s)\right\|_{H_{\varepsilon}}^{2} d s \\
&+C\left(1+t^{2}\right)\left(1+k\left(r_{0}\right)\right) \int_{0}^{t}\left\|u^{\varepsilon}(s)-v(s)\right\|_{H_{\varepsilon}^{1}(Q)}^{2} d s \\
&+\tilde{B}_{\varepsilon}^{4}(t)+\tilde{B}_{\varepsilon}^{5}(t)+\tilde{B}_{\varepsilon}^{6}(t) .
\end{aligned}
$$

Arguing as in the proof of the estimate (6.11), one can show that, for any positive number $\delta$, for $t>0$,

$$
\tilde{B}_{\varepsilon}^{4}(t) \leqq \delta\left\|z^{\varepsilon}(t)-z^{0}(t)\right\|_{V_{\varepsilon}}^{2}+C \varepsilon\left(1+\delta^{-1}\right)\left(t+t^{2}\right) k\left(r_{0}\right) .
$$

Arguing now as in the proof of the estimate (6.14), we obtain, for any positive number $\delta$, for $t>0$,

$$
\begin{aligned}
\tilde{B}_{\varepsilon}^{5}(t) & \leqq \delta\left\|z^{\varepsilon}(t)-z^{0}(t)\right\|_{V_{\varepsilon}}^{2}+C \varepsilon\left(1+\delta^{-1}\right) t^{2}\|v\|_{H^{2}((0,1)) \times H^{2}((0,1))}^{2} \\
& \leqq \delta\left\|z^{\varepsilon}(t)-z^{0}(t)\right\|_{V_{\varepsilon}}^{2}+C \varepsilon\left(1+\delta^{-1}\right)\left(1+t^{2}\right) k\left(r_{0}\right) .
\end{aligned}
$$

Finally, using Lemma 2.3 and the estimate (6.3), we have, for any positive number $\delta$, for $t>0$,

$$
\begin{aligned}
\tilde{B}_{\varepsilon}^{6}(t) & \leqq \delta\left\|z^{\varepsilon}(t)-z^{0}(t)\right\|_{V_{\varepsilon}}^{2}+C \varepsilon\left(1+\delta^{-1}\right) t^{2}\|v\|_{H^{2}((0,1)) \times H^{2}((0,1))}^{2} \\
& \leqq \delta\left\|z^{\varepsilon}(t)-z^{0}(t)\right\|_{V_{\varepsilon}}^{2}+C \varepsilon\left(1+\delta^{-1}\right)\left(1+t^{2}\right) k\left(r_{0}\right) .
\end{aligned}
$$

Choosing $\delta$ sufficiently small, we at once derive from the inequalities (6.20), (7.16)-(7.19), the following estimate for $t \geqq 0$ :

$$
\int_{0}^{t} s^{2}\left\|u_{t}^{\varepsilon}(s)-v_{t}(s)\right\|_{H_{\varepsilon}}^{2} d s+t^{2}\left\|u^{\varepsilon}(t)-v(t)\right\|_{H_{\varepsilon}^{1}(Q)}^{2} \leqq C \varepsilon k\left(r_{0}\right) e^{k\left(r_{0}\right) t}
$$

\section{Comparison of equilibrium points of the problems $(P)_{\varepsilon}$ and $(P)_{0}$}

Since we are interested at the beginning of this section only in local results, it is not necessary to assume that the condition (2.2) holds. We recall that $A_{\varepsilon}^{-1} \in \mathscr{L}\left(\mathscr{H}_{\varepsilon}, \mathscr{V}_{\varepsilon}\right)$ 
(respectively $A_{0}^{-1} \in \mathscr{L}\left(\mathscr{H}_{0}, \mathscr{V}_{0}\right)$ ) is the operator defined for $\hat{h}_{\varepsilon} \in \mathscr{H}_{\varepsilon}$ by

$$
A_{\varepsilon}^{-1} \hat{h}_{\varepsilon}=u^{\varepsilon} \quad \text { if and only if } a_{\varepsilon}\left(u^{\varepsilon}, w\right)=\left(\hat{h}_{\varepsilon}, w\right)_{H_{\varepsilon}} \forall w \in \mathscr{V}_{\varepsilon}
$$

(respectively, for $\hat{h}_{0} \in L^{2}((0,1)) \times L^{2}((0,1))$,

$$
\left.A_{0}^{-1} \hat{h}_{0}=v \quad \text { if and only if } a_{0}(v, \xi)=\left(\hat{h}_{0}, \xi\right)_{H_{0}} \forall \xi \in \mathscr{V}_{0}\right) \text {. }
$$

Moreover, for $0<\varepsilon \leqq \varepsilon_{0}$, we have (see Section 2),

$$
\left\|A_{\varepsilon}^{-1} \hat{h}_{\varepsilon}\right\|_{\mathscr{r}_{\varepsilon}} \leqq C\left\|\hat{h}_{\varepsilon}\right\|_{H_{\varepsilon}}
$$

and

$$
\max \left(\left\|A_{0}^{-1} \hat{h}_{0}\right\|_{H^{2}((0,1)) \times H^{2}((0,1))},\left\|A_{0}^{-1} \hat{h}_{0}\right\|_{\mathscr{r}_{0}}\right) \leqq C\left\|\hat{h}_{0}\right\|_{L^{2}((0,1)) \times L^{2}((0,1))} .
$$

We need the following auxiliary result.

LEMMA 8.1. For $0 \leqq \varepsilon \leqq \varepsilon_{0}$, we have the following estimate, for any $\hat{h}_{\varepsilon} \in \mathscr{H}_{\varepsilon}$, $\hat{h}_{0} \in L^{2}((0,1)) \times L^{2}((0,1))$,

$$
\begin{aligned}
\max \left(\left\|A_{\varepsilon}^{-1} \hat{h}_{\varepsilon}-A_{0}^{-1} \hat{h}_{0}\right\|_{H_{\varepsilon}^{1}(Q)},\left\|A_{\varepsilon}^{-1} \hat{h}_{\varepsilon}-A_{0}^{-1} \hat{h}_{0}\right\|_{V_{\varepsilon}}\right) \\
\leqq C\left[\left\|\hat{h}_{\varepsilon}-\hat{h}_{0}\right\|_{H_{\varepsilon}}+\varepsilon^{\frac{1}{2}}\left(\left\|\hat{h}_{\varepsilon}\right\|_{H_{\varepsilon}}+\left\|\hat{h}_{0}\right\|_{H_{\varepsilon}}\right)\right] .
\end{aligned}
$$

Proof. Let $u^{\varepsilon}=A_{i}^{-1} \hat{h}_{\varepsilon}$ and $v=A_{0}^{-1} \hat{h}_{0}$. As in Section 6, we can write:

$$
a_{\varepsilon}\left(u^{\varepsilon}-v, u^{\varepsilon}-v\right)=\left(\hat{h}_{\varepsilon}-\hat{h}_{0}, u^{\varepsilon}-v\right)_{H_{\varepsilon}}+\bar{B}_{\varepsilon}^{1}\left(u^{\varepsilon}, v\right)+B_{\varepsilon}^{2}\left(u^{\varepsilon}, v\right),
$$

where $B_{\varepsilon}^{2}\left(u^{\varepsilon}, v\right)$ is given by $(6.2 \mathrm{c})$ and

$$
\begin{aligned}
\bar{B}_{\varepsilon}^{1}\left(u^{\varepsilon}, v\right)= & \left(\hat{h}_{\varepsilon}, v-I_{\varepsilon} v\right)_{H_{2 \varepsilon M}}+\left(\hat{h}_{0}, u^{\varepsilon}-M_{\varepsilon} u^{\varepsilon}\right)_{H_{2 \varepsilon M}} \\
& +\frac{1}{2} \sum_{j=1}^{2} \int_{J_{\varepsilon}^{j}} g_{j} h_{0 j}\left(v_{j}-u_{j}^{\varepsilon}\right) d x_{1} d x_{2} \\
& +\frac{1}{2} \sum_{j=1}^{2} \int_{J_{\varepsilon}^{j}} g_{j} h_{\varepsilon j}\left(v_{j}(0)-v_{j}\right) d x_{1} d x_{2} .
\end{aligned}
$$

Arguing as in Section 6 and using the estimates $(2.4)_{\varepsilon}$, we obtain, for $\delta>0$ small enough,

$$
\begin{aligned}
& \left\|u^{\varepsilon}-v\right\|_{H_{\varepsilon}^{1}(Q)}^{2} \leqq \delta\left\|u^{\varepsilon}-v\right\|_{H_{\varepsilon}^{1}(Q)}^{2}+C \delta^{-1}\left\|\hat{h}_{\varepsilon}-\hat{h}_{0}\right\|_{H_{\varepsilon}}^{2} \\
& \quad+C \varepsilon\left(1+\delta^{-1}\right)\left[\left\|\hat{h}_{0}\right\|_{H_{\varepsilon}}^{2}+\left\|\hat{h}_{0}\right\|_{H_{2 \varepsilon M}}\left\|\hat{h}_{\varepsilon}\right\|_{H_{\varepsilon}}+\varepsilon^{\frac{1}{2}}\left\|\hat{h}_{0}\right\|_{H_{\varepsilon}}\left\|\hat{h}_{\varepsilon}\right\|_{H_{2 \varepsilon M}}\right],
\end{aligned}
$$

which implies that

$$
\begin{aligned}
\left\|u^{\varepsilon}-v\right\|_{H_{\varepsilon}^{1}(Q)}^{2} & \leqq C\left\|\hat{h}_{\varepsilon}-\hat{h}_{0}\right\|_{H_{\varepsilon}}^{2} \\
& +C \varepsilon\left[\left\|\hat{h}_{0}\right\|_{H_{\varepsilon}}^{2}+\left\|\hat{h}_{0}\right\|_{H_{2 \varepsilon M}}\left\|\hat{h}_{\varepsilon}\right\|_{H_{\varepsilon}}+\varepsilon^{\frac{1}{2}}\left\|\hat{h}_{0}\right\|_{H_{\varepsilon}}\left\|\hat{h}_{\varepsilon}\right\|_{H_{2 \varepsilon M}}\right] .
\end{aligned}
$$

From the inequalities (8.6) and $(2.4)_{\varepsilon}$, we deduce in particular Lemma 8.1.

We assume now that $v_{0} \in \mathscr{V}_{0}$ is a hyperbolic equilibrium point of the problem $(\tilde{\mathbf{P}})_{0}$ or $(\mathrm{P})_{0}$. Since the differential equation defines a gradient system, this is equivalent 
to: $v_{0} \in \mathscr{V}_{0} \cap\left(H^{2}((0,1)) \times H^{2}((0,1))\right)$ is a solution of the equation

$$
F_{0}(v) \equiv v+A_{0}^{-1}\left(\hat{f}(v)+\hat{G}_{0}\right)=0
$$

and the operator $I+A_{0}^{-1} D \hat{f}\left(v_{0}\right) \in \mathscr{L}\left(\mathscr{V}_{0} ; \mathscr{V}_{0}\right)$ is an isomorphism of $\mathscr{V}_{0}$. We set $r_{0}=\left\|v_{0}\right\|_{H^{1}((0,1)) \times H^{1}((0,1))}$.

We want to show that there exist positive constants $r_{1}$ and $\varepsilon_{1}$ such that, for $0<\varepsilon \leqq \varepsilon_{1}$, the equation

$$
F_{\varepsilon}(u) \equiv u+A_{\varepsilon}^{-1}\left(\hat{f}(u)+\hat{G}_{\varepsilon}\right)=0
$$

has a unique solution $u$ in $\mathscr{V}_{\varepsilon} \cap B_{H_{\varepsilon}^{1}(Q)}\left(v_{0} ; r_{1}\right)$, where $B_{H_{\varepsilon}^{1}(Q)}\left(v_{0} ; r_{1}\right)$ denotes the closed ball of centre $v_{0}$ and radius $r_{1}$ in $H_{\varepsilon}^{1}(Q)$. To this end, as in [13] or in [29], we apply a particular form of the contraction mapping theorem (see [8]). For this reason, we are going to estimate $F_{\varepsilon}\left(I_{\varepsilon} v_{0}\right)$ and to show that $D F_{\varepsilon}\left(I_{\varepsilon} v_{0}\right) \in \mathscr{L}\left(\mathscr{V}_{\varepsilon} ; \mathscr{W}_{\varepsilon}\right)$ is an isomorphism on $\mathscr{V}_{\varepsilon}$.

We set $\delta_{\varepsilon}=\left\|F_{\varepsilon}\left(I_{\varepsilon} v_{0}\right)\right\|_{H_{\varepsilon}^{1}(Q)}$. Applying Lemma 8.1 and using the estimate (3.3c) as well as the hypothesis (2.1), we obtain, for $0<\varepsilon \leqq \varepsilon_{0}$,

$$
\begin{aligned}
\delta_{\varepsilon} \leqq \| v_{0}- & I_{\varepsilon} v_{0}\left\|_{H_{\varepsilon}^{1}(Q)}+C\right\| \hat{f}\left(I_{\varepsilon} v_{0}\right)-\hat{f}\left(v_{0}\right) \|_{H_{\varepsilon}} \\
& +C\left\|\hat{G}_{\varepsilon}-\hat{G}_{0}\right\|_{H_{\varepsilon}}+C \varepsilon^{\frac{1}{2}}\left(\left\|\hat{f}\left(I_{\varepsilon} v_{0}\right)+\hat{G}_{\varepsilon}\right\|_{H_{\varepsilon}}+\left\|\hat{f}\left(v_{0}\right)+\hat{G}_{0}\right\|_{H_{\varepsilon}}\right)
\end{aligned}
$$

or

$$
\delta_{\varepsilon} \leqq C\left(r_{0}\right) \varepsilon^{\frac{1}{2}},
$$

where $C\left(r_{0}\right)$ is a positive constant depending only on $r_{0}$.

We now show that $D F_{\varepsilon}\left(I_{\varepsilon} v_{0}\right)$ is invertible on $\mathscr{V}_{\varepsilon}$ and obtain an estimate of the quantity $\left\|\left(D F_{\varepsilon}\left(I_{\varepsilon} v_{0}\right)\right)^{-1}\right\|_{\mathscr{L}\left(\gamma_{\varepsilon} ; r_{\varepsilon}\right)}$. We can write

$$
\begin{aligned}
D F_{\varepsilon}\left(I_{\varepsilon} v_{0}\right) w= & w+I_{\varepsilon} A_{0}^{-1} D \hat{f}\left(v_{0}\right) M_{\varepsilon} w \\
& +\left(A_{\varepsilon}^{-1} D \hat{f}\left(I_{\varepsilon} v_{0}\right) w-I_{\varepsilon} A_{0}^{-1} D \hat{f}\left(v_{0}\right) M_{\varepsilon} w\right) .
\end{aligned}
$$

Applying Lemma 8.1 again and using the estimate (3.3c) as well as the hypothesis (2.1), we have, for $0<\varepsilon \leqq \varepsilon_{0}$,

$$
\begin{aligned}
&\left\|A_{\varepsilon}^{-1} D \hat{f}\left(I_{\varepsilon} v_{0}\right) w-I_{\varepsilon} A_{0}^{-1} D \hat{f}\left(v_{0}\right) M_{\varepsilon} w\right\|_{H_{\varepsilon}^{1}(Q)} \\
& \leqq\left\|A_{\varepsilon}^{-1} D \hat{f}\left(I_{\varepsilon} v_{0}\right) w-A_{0}^{-1} D \hat{f}\left(v_{0}\right) M_{\varepsilon} w\right\|_{H_{\varepsilon}^{1}(Q)}+\left\|\left(I-I_{\varepsilon}\right) A_{0}^{-1} D \hat{f}\left(v_{0}\right) M_{\varepsilon} w\right\|_{H_{\varepsilon}^{1}(Q)} \\
& \leqq C\left[\varepsilon^{\frac{1}{2}}\left(\left\|D \hat{f}\left(I_{\varepsilon} v_{0}\right) w\right\|_{H_{\varepsilon}}+\left\|D \hat{f}\left(v_{0}\right) M_{\varepsilon} w\right\|_{H_{\varepsilon}}\right)+\left\|D \hat{f}\left(I_{\varepsilon} v_{0}\right)\left(w-M_{\varepsilon} w\right)\right\|_{H_{\varepsilon}}\right. \\
&\left.+\left\|\left(D \hat{f}\left(I_{\varepsilon} v_{0}\right)-D \hat{f}\left(v_{0}\right)\right) M_{\varepsilon} w\right\|_{H_{\varepsilon}}+C \varepsilon^{\frac{1}{2}}\left\|D \hat{f}\left(v_{0}\right) M_{\varepsilon} w\right\|_{H_{\varepsilon}}\right],
\end{aligned}
$$

which implies that

$$
\left\|A_{\varepsilon}^{-1} D \hat{f}\left(I_{\varepsilon} v_{0}\right) w-I_{\varepsilon} A_{0}^{-1} D \hat{f}\left(v_{0}\right) M_{\varepsilon} w\right\|_{H_{\varepsilon}^{1}(Q)} \leqq C\left(r_{0}\right) \varepsilon^{\frac{1}{2}}\|w\|_{H_{\varepsilon}^{1}(Q)} .
$$

It remains to show that $I+I_{\varepsilon} A_{0}^{-1} D \hat{f}\left(v_{0}\right) M_{\varepsilon}$ is an isomorphism from $\mathscr{V}_{\varepsilon}$ onto $\mathscr{V}_{\varepsilon}$. At first, we remark that, for any $v$ in $\mathscr{V}_{0}$,

$$
\left(I+M_{\varepsilon} I_{\varepsilon} A_{0}^{-1} D \hat{f}\left(v_{0}\right)\right) v=\left(I+A_{0}^{-1}\left(D \hat{f}\left(v_{0}\right)\right)\right) v+\left(\left(M_{\varepsilon} I_{\varepsilon}-I\right) A_{0}^{-1}\left(D \hat{f}\left(v_{0}\right)\right)\right) v .
$$

Since, thanks to Remark 3.2,

$$
\begin{aligned}
\left\|\left(\left(M_{\varepsilon} I_{\varepsilon}-I\right) A_{0}^{-1}\left(D \hat{f}\left(v_{0}\right)\right)\right) v\right\|_{\mathscr{r}_{0}} & \leqq C \varepsilon^{\frac{1}{2}}\left\|A_{0}^{-1}\left(D \hat{f}\left(v_{0}\right)\right) v\right\|_{H^{2}((0,1)) \times H^{2}((0,1))} \\
& \leqq C\left(r_{0}\right) \varepsilon^{\frac{1}{2}}\|v\|_{\mathscr{V}_{0}},
\end{aligned}
$$


we deduce from the equality (8.11) that $I+M_{\varepsilon} I_{\varepsilon} A_{0}^{-1} D \hat{f}\left(v_{0}\right)$ is an isomorphism of $\mathscr{V}_{0}$, and there exists a positive constant $K_{0}$, independent of $\varepsilon$, such that, for $0<\varepsilon \leqq \varepsilon_{0}$,

$$
\left\|\left(I+M_{\varepsilon} I_{\varepsilon} A_{0}^{-1} D \hat{f}\left(v_{0}\right)\right)^{-1}\right\|_{\mathscr{L}\left(\mathscr{r}_{0} ; \mathscr{r}_{0}\right)} \leqq K_{0}
$$

Now let $U_{\varepsilon}$ be an element of $\mathscr{V}_{\varepsilon}$. We want to show that there exists a unique element $w_{\varepsilon}$ in $\mathscr{V}_{\varepsilon}$ such that

$$
U_{\varepsilon}=w_{\varepsilon}+I_{\varepsilon} A_{0}^{-1} D \hat{f}\left(v_{0}\right) M_{\varepsilon} w_{\varepsilon} .
$$

We note that (8.14) is equivalent to the system

$$
\begin{aligned}
& \text { (i) } M_{\varepsilon} U_{\varepsilon}=M_{\varepsilon} w_{\varepsilon}+M_{\varepsilon} I_{\varepsilon} A_{0}^{-1} D \hat{f}\left(v_{0}\right) M_{\varepsilon} w_{\varepsilon}, \\
& \text { (ii) }\left(I-M_{\varepsilon}\right) U_{\varepsilon}=\left(I-M_{\varepsilon}\right) w_{\varepsilon}+\left(I-M_{\varepsilon}\right) I_{\varepsilon} A_{0}^{-1} D \hat{f}\left(v_{0}\right) M_{\varepsilon} w_{\varepsilon} \text {. }
\end{aligned}
$$

Since $I+M_{\varepsilon} I_{\varepsilon} A_{0}^{-1} D \hat{f}\left(v_{0}\right)$ is an isomorphism of $\mathscr{V}_{0}$, there exists a unique $v_{1}$ in $\mathscr{V}_{0}$ such that

$$
M_{\varepsilon} U_{\varepsilon}=v_{1}+M_{\varepsilon} I_{\varepsilon} A_{0}^{-1} D \hat{f}\left(v_{0}\right) v_{1}
$$

and, by (8.13),

$$
\left\|v_{1}\right\|_{r_{0}} \leqq K_{0}\left\|M_{\varepsilon} U_{\varepsilon}\right\|_{r_{0}} \leqq C K_{0}\left\|U_{\varepsilon}\right\|_{H_{\varepsilon}^{1}(Q)}
$$

We set

$$
w_{\varepsilon}=U_{\varepsilon}-I_{\varepsilon} A_{0}^{-1} D \hat{f}\left(v_{0}\right) v_{1} .
$$

From (8.16) and (8.18), we derive that

$$
M_{\varepsilon} w_{\varepsilon}=M_{\varepsilon} U_{\varepsilon}-M_{\varepsilon} I_{\varepsilon} A_{0}^{-1} D \hat{f}\left(v_{0}\right) v_{1}=v_{1},
$$

which implies, by (8.18),

$$
U_{\varepsilon}=w_{\varepsilon}+I_{\varepsilon} A_{0}^{-1} D \hat{f}\left(v_{0}\right) M_{\varepsilon} w_{\varepsilon} .
$$

From (8.17) and (8.18), we at once infer that

$$
\left\|w_{\varepsilon}\right\|_{H_{\varepsilon}^{1}(Q)} \leqq C\left(1+K_{0} C\left(r_{0}\right)\right)\left\|U_{\varepsilon}\right\|_{H_{\varepsilon}^{1}(Q)} .
$$

In a similar way, one shows that, if $U_{\varepsilon}=0$, then $w_{\varepsilon}=0$. We thus have shown that $D F_{\varepsilon}\left(I_{\varepsilon} v_{0}\right)$ is an isomorphism of $\mathscr{V}_{\varepsilon}$ and that, for $0<\varepsilon \leqq \varepsilon_{0}$,

$$
\left\|\left(D F_{\varepsilon}\left(I_{\varepsilon} v_{0}\right)\right)^{-1}\right\|_{\mathscr{L}\left(\mathscr{r}_{\varepsilon} ; \mathscr{r}_{\varepsilon}\right)} \leqq K_{1},
$$

where $K_{1}$ is a positive constant independent of $\varepsilon$.

Using the hypothesis (2.1), one directly shows that, for $0<\varepsilon \leqq \varepsilon_{0}$, for $0<\theta \leqq \theta_{0}$,

$$
\begin{aligned}
& \sup _{u \in B \mathcal{V}_{\varepsilon}\left(I_{\varepsilon} v_{0} ; \theta\right)}\left\|\left(D F_{\varepsilon}\left(I_{\varepsilon} v_{0}\right)-D F_{\varepsilon}(u)\right) w\right\|_{H_{\varepsilon}^{1}(Q)} \leqq C\left\|\left(D \hat{f}\left(I_{\varepsilon} v_{0}\right)-D \hat{f}(u)\right) w\right\|_{H_{\varepsilon}} \\
& \leqq C \theta\left(C\left(r_{0}\right)+C\left(r_{0}+\theta_{0}\right)\right)\|w\|_{H_{\varepsilon}^{1}(Q)},
\end{aligned}
$$

or

$$
\begin{aligned}
l_{\varepsilon}(\theta) & \equiv \sup _{u \in B \gamma_{\varepsilon}\left(I_{\varepsilon} v_{0} ; \theta\right)}\left\|D F_{\varepsilon}\left(I_{\varepsilon} v_{0}\right)-D F_{\varepsilon}(u)\right\|_{\mathscr{L}\left(\mathscr{V}_{\varepsilon} ; r_{\varepsilon}\right)} \\
& \leqq K_{2} \theta C\left(r_{0}+\theta_{0}\right),
\end{aligned}
$$

where $K_{2}$ is a positive constant independent of $\varepsilon$. 
The contraction mapping is as given in [8, Theorem 3.1] asserts the following: if $2 K_{1} l_{\varepsilon}\left(2 K_{1} \delta_{\varepsilon}\right)<1$, then, for any $\theta \geqq 2 K_{1} \delta_{\varepsilon}$ such that $K_{1} l_{\varepsilon}(\theta)<1$, the equation $(8.7)_{\varepsilon}$ has a unique solution $u_{0}^{\varepsilon}$ in $B_{H_{\varepsilon}^{1}(Q)}\left(I_{\varepsilon} v_{0} ; \theta\right) \cap \mathscr{V}_{\varepsilon}$. Moreover,

$$
\left\|\left(D F_{\varepsilon}\left(u_{0}^{\varepsilon}\right)\right)^{-1}\right\|_{\mathscr{L}\left(\mathscr{V}_{\varepsilon} ; \mathscr{V}_{\varepsilon}\right)} \leqq 2 K_{1}
$$

and

$$
\left\|u_{0}^{\varepsilon}-I_{\varepsilon} v_{0}\right\|_{H_{\varepsilon}^{1}(Q)} \leqq K_{1} \delta_{\varepsilon}
$$

From this assertion as well as from the estimates (8.8), (8.22) and (8.23), we deduce the following result:

THEOREM 8.2. Assume that $v_{0} \in \mathscr{V}_{0}$ is a hyperbolic equilibrium point of $(\mathrm{P})_{0}$. Then there exist positive constants $\varepsilon_{1}$ and $r_{1}$ such that, for $0<\varepsilon \leqq \varepsilon_{1}$, the equation $(8.7)_{\varepsilon}$ has $a$ unique solution $u_{0}^{\varepsilon}$ in $B_{H_{\varepsilon}^{1}(Q)}\left(v_{0} ; r_{1}\right) \cap \mathscr{V}_{\varepsilon}$. Moreover, we have

$$
\left\|\left(D F_{\varepsilon}\left(u_{0}^{\varepsilon}\right)\right)^{-1}\right\|_{\mathscr{L}\left(\mathscr{V}_{\varepsilon} ; \mathscr{V}_{\varepsilon}\right)} \leqq 2 K_{1}
$$

and

$$
\left\|u_{0}^{\varepsilon}-v_{0}\right\|_{H_{\varepsilon}^{1}(Q)} \leqq C \varepsilon^{\frac{1}{2}} .
$$

From Theorem 8.2, we easily deduce the following global result. Let $E_{0}$ (respectively $E_{\varepsilon}$ ) denote the set of equilibrium points of $(\mathrm{P})_{0}\left(\right.$ respectively $\left.(\mathrm{P})_{\varepsilon}\right)$.

Corollary 8.3. Assume that the conditions (2.1) and (2.2) hold and that all of the equilibrium points of $(\mathrm{P})_{0}$ are hyperbolic. Then the set $E_{0}$ is finite and contains say, $N_{0}$ elements, $v_{j}, 1 \leqq j \leqq N_{0}$. Moreover, there exist positive constants $\varepsilon_{0}^{*}$ and $r_{0}^{*}$ such that, for $0<\varepsilon \leqq \varepsilon_{0}^{*}$, the set $E_{\varepsilon}$ contains exactly $N_{0}$ elements $u_{j}^{\varepsilon}, 1 \leqq j \leqq N_{0}$, and, furthermore, the following inequalities are satisfied:

$$
\left\|u_{j}^{\varepsilon}-v_{j}\right\|_{H_{\varepsilon}^{1}(Q)} \leqq C \varepsilon^{\frac{1}{2}} \leqq r_{0}^{*} .
$$

In addition, each element $u_{j}^{\varepsilon}$ is hyperbolic.

Proof. The fact that $E_{0}$ is finite is obvious. Applying Theorem 8.2 at each hyperbolic equilibrium point $v_{j}$ yields that there exist positive constants $\varepsilon^{*}$ and $r_{0}^{*}$ such that, for $0<\varepsilon \leqq \varepsilon^{*}, E_{\varepsilon} \cap B_{H_{\varepsilon}^{1}(Q)}\left(v_{0} ; r_{0}^{*}\right)$ contains a unique equilibrium point $u_{j}^{\varepsilon}$ and (8.28) holds. It remains to show that there exists a positive constant $\varepsilon_{0}^{*}$ such that, for $0<\varepsilon \leqq \varepsilon_{0}^{*}, E_{\varepsilon}$ contains exactly the $N_{0}$ equilibria $u_{j}^{\varepsilon}, 1 \leqq j \leqq N_{0}$. Assume that there exist two sequences $\varepsilon_{n}$ and $u_{\varepsilon_{n}}$ such that

$$
\lim _{n \rightarrow \infty} \varepsilon_{n}=0, \quad u_{\varepsilon_{n}} \in E_{\varepsilon_{n}} \backslash \bigcup_{j} B_{H_{\varepsilon_{n}}^{1}(Q)}\left(v_{j}, r_{0}^{*}\right) \text {. }
$$

Since $u_{\varepsilon_{n}}$ belongs to the attractor $\mathscr{A}_{\varepsilon_{n}}$, we at once show, by using the uppersemicontinuity of the attractors $\mathscr{A}_{\varepsilon_{n}}$ and the compactness of $\mathscr{A}_{0}$, that there exists a subsequence $u_{\varepsilon_{n_{k}}}$ converging in $H_{\varepsilon_{n_{k}}}^{1}(Q)$ to an element $v_{0} \in \mathscr{A}_{0}$. Using this fact and Lemma 8.1, we conclude that $v_{0}$ belongs to $E_{0}$, which contradicts the hypothesis (8.29).

\section{Comparison of eigenvalues}

Before comparing the eigenvalues of $A_{0}+D \hat{f}\left(v_{0}\right)$ with those of $A_{\varepsilon}+D \hat{f}\left(u_{0}^{\varepsilon}\right)$ for $\left\|v_{0}-u_{0}^{\varepsilon}\right\|_{H_{\varepsilon}^{1}(Q)}$ small enough, we present a more general version of comparison of eigenvalues which is an extension of the results of Hale and Raugel [14]. For related results, see also $[\mathbf{2 0}, \mathbf{2 7}]$. 


\section{A semi-abstract version}

We keep the above spaces $\mathscr{V}_{\varepsilon}, \mathscr{H}_{\varepsilon}, \mathscr{V}_{0}, H_{\varepsilon}^{1}(Q), H_{\varepsilon}, \ldots$, and we introduce linear operators $B_{0}$ and $B_{\varepsilon}, 0<\varepsilon \leqq \varepsilon_{0}$, which satisfy

$$
\begin{aligned}
& B_{0} \in \mathscr{L}\left(\mathscr{V}_{0} ; \mathscr{V}_{0}\right) \cap \mathscr{L}\left(L^{2}((0,1)) \times L^{2}((0,1)) ;\left(H^{2}((0,1)) \times H^{2}((0,1))\right) \cap \mathscr{V}_{0}\right) \\
& B_{\varepsilon} \in \mathscr{L}\left(\mathscr{V}_{\varepsilon} ; \mathscr{V}_{\varepsilon}\right) \cap \mathscr{L}\left(\mathscr{H}_{\varepsilon} ; \mathscr{V}_{\varepsilon}\right)
\end{aligned}
$$

(in particular, $B_{0}: \mathscr{V}_{0} \rightarrow \mathscr{V}_{0}$ is compact) as well as the following inequalities for positive constants $C_{1}, \alpha$, independent of $\varepsilon$ :

$$
\left\|B_{\varepsilon}\right\|_{\mathscr{L}\left(\mathscr{H}_{\varepsilon} ; \mathscr{V}_{\varepsilon}\right)}+\left\|B_{0}\right\|_{\mathscr{L}\left(L^{2}((0,1)) \times L^{2}((0,1)) ;\left(H^{2}((0,1)) \times H^{2}((0,1))\right) \cap \mathscr{r}_{0}\right)} \leqq C_{1},
$$

and, for any $\hat{h}_{\varepsilon} \in \mathscr{H}_{\varepsilon}, \hat{h}_{0} \in L^{2}((0,1)) \times L^{2}((0,1))$,

$$
\left\|B_{\varepsilon} \hat{h}_{\varepsilon}-B_{0} \hat{h}_{0}\right\|_{H_{\varepsilon}^{1}(Q)} \leqq C_{1}\left[\left\|\hat{h}_{\varepsilon}-\hat{h}_{0}\right\|_{H_{\varepsilon}}+\varepsilon^{\alpha}\left(\left\|\hat{h}_{\varepsilon}\right\|_{H_{\varepsilon}}+\left\|\hat{h}_{0}\right\|_{H_{\varepsilon}}\right)\right] \text {. }
$$

In all of the results below, we constantly use the equivalence of the norms $\|\cdot\|_{H_{\varepsilon}^{1}(Q)}$ and $\|\cdot\|_{V_{\varepsilon}}$ as stated in $(2.4)_{\varepsilon}$, without recalling it. For any operator $B$, we denote by $\sigma(B)$ the spectrum of $B$. In the following, a closed curve in $\mathbb{C}$ denotes a homeomorphic image of a circle. Also, for any $\eta>0$ and $\lambda \in \mathbb{C}$, let $B(\lambda, \eta)$ be the disk of radius $\eta$ and centre $\lambda$. Since $B_{0}$ is compact, for any $\eta>0$, there is an integer $p=p(\eta)$ and distinct complex numbers $\lambda_{1}, \ldots, \lambda_{p}$ such that

$$
\sigma_{\eta}\left(B_{0}\right) \equiv \sigma\left(B_{0}\right) \cap(\mathbb{C} \backslash B(0, \eta))=\left\{\lambda_{1}, \ldots, \lambda_{p}\right\}
$$

Moreover, there exist a compact set $\tilde{K}$ with $0 \in \tilde{K}$ and a positive constant $C_{2}$ such that $\sigma\left(B_{0}\right) \cap(\mathbb{C} \backslash \tilde{K})=\varnothing$ and

$$
\sup _{\lambda \in(\mathbb{C}(\tilde{K})}\left\|\left(\lambda I-B_{0}\right)^{-1}\right\|_{\mathscr{L}\left(H_{0} ; H_{0}\right)}+\sup _{\lambda \in(\mathbb{C} \backslash \tilde{K})}\left\|\left(\lambda I-B_{0}\right)^{-1}\right\|_{\mathscr{L}\left(\mathscr{r}_{0} ; r_{0}\right)} \leqq C_{2}
$$

We need the following lemma (see [14, Lemmas 3.1 and $3.1 \mathrm{bis}]$ ).

LEMMA 9.1. There exist positive constants $\tilde{C}$ and $\tilde{\varepsilon}, 0<\tilde{\varepsilon} \leqq \varepsilon_{0}$, such that, for $0<\varepsilon \leqq \tilde{\varepsilon}$, we have

$$
\begin{gathered}
\left\|B_{\varepsilon}-I_{\varepsilon} B_{0} M_{\varepsilon}\right\|_{\mathscr{L}\left(\mathscr{V}_{\varepsilon} ; \mathscr{V}_{\varepsilon}\right)} \leqq \tilde{C}\left(\varepsilon^{\alpha}+\varepsilon^{\frac{1}{2}}\right), \\
\left\|B_{0}-I_{\varepsilon} B_{0} M_{\varepsilon}\right\|_{\mathscr{L}\left(\mathscr{V}_{0} ; \mathscr{V}_{0}\right)}+\left\|B_{0}-M_{\varepsilon} I_{\varepsilon} B_{0}\right\|_{\mathscr{L}\left(\mathscr{V}_{0} ; \mathscr{V}_{0}\right)} \leqq \tilde{C} \varepsilon^{\frac{1}{2}}
\end{gathered}
$$

and, on $\tilde{U}=\mathbb{C} \backslash \tilde{K}$,

$$
\begin{gathered}
\sup _{\lambda \in \tilde{U}}\left\|\left(\lambda I-I_{\varepsilon} B_{0} M_{\varepsilon}\right)^{-1}\right\|_{\mathscr{L}\left(\mathscr{V}_{\varepsilon} ; \mathscr{V}_{\varepsilon}\right) \leqq \tilde{C},} \\
\sup _{\lambda \in \tilde{U}}\left\|\left(\lambda I-B_{\varepsilon}\right)^{-1}\right\|_{\mathscr{L}\left(\mathscr{V}_{\varepsilon} ; \mathscr{V}_{\varepsilon}\right) \leqq \tilde{C},} \\
\sup _{\lambda \in \tilde{U}}\left\|\left(\lambda I-I_{\varepsilon} B_{0} M_{\varepsilon}\right)^{-1}\right\|_{\mathscr{L}\left(\mathscr{V}_{0} ; \mathscr{V}_{0}\right)} \leqq \tilde{C}, \\
\sup _{\lambda \in \tilde{U}}\left\|\left(\lambda I-B_{\varepsilon}\right)^{-1}-\left(\lambda I-I_{\varepsilon} B_{0} M_{\varepsilon}\right)^{-1}\right\|_{\mathscr{L}_{\left(\mathscr{V}_{\varepsilon} ; \mathscr{V}_{\varepsilon}\right)} \leqq \tilde{C}\left(\varepsilon^{\alpha}+\varepsilon^{\frac{1}{2}}\right),} \\
\sup _{\lambda \in \tilde{U}}\left\|\left(\lambda I-B_{0}\right)^{-1}-\left(\lambda I-I_{\varepsilon} B_{0} M_{\varepsilon}\right)^{-1}\right\|_{\mathscr{L}\left(\mathscr{V}_{0} ; \mathscr{V}_{0}\right)} \leqq \tilde{C} \varepsilon^{\frac{1}{2}}, \\
\sup _{\lambda \in \tilde{U}}\left\|\left(\lambda I-B_{0}\right)^{-1}-\left(\lambda I-M_{\varepsilon} I_{\varepsilon} B_{0}\right)^{-1}\right\|_{\mathscr{L}\left(\mathscr{V}_{0} ; \mathscr{V}_{0}\right)} \leqq \tilde{C} \varepsilon^{\frac{1}{2}},
\end{gathered}
$$

Arguing as in the proof of Lemma 9.1, one can show the following local result: 
LEMMA 9.1'. For any compact set $K$ in the resolvent set of $B_{0}$ with $0 \notin K$, there are positive constants $C_{K}, \varepsilon_{K}$ such that, for $0<\varepsilon \leqq \varepsilon_{K}$, the estimates (9.8)-(9.13) (respectively the estimate (9.16) below) hold with $\sup _{\lambda \in \tilde{U}}$ replaced by $\sup _{\lambda \in K}$ and $\tilde{C}$ (respectively $C_{3}$ ) replaced by $C_{K}$.

The proof of Lemma 9.1 uses techniques and ideas contained in Section 8. In the sequel, we often use the facts that $I_{\varepsilon} \in \mathscr{L}\left(\mathscr{V}_{0} ; \mathscr{V}_{0} \cap \mathscr{V}_{\varepsilon}\right)$ and $M_{\varepsilon} \in \mathscr{L}\left(\mathscr{Y}_{0} ; H_{0}\right)$.

Proof of Lemma 9.1. The estimate (9.6) is a direct consequence of (9.3) and the estimates (3.3c) and (3.6). Indeed, for any $w \in \mathscr{V}_{\varepsilon}$,

$$
\begin{aligned}
\left\|\left(B_{\varepsilon}-I_{\varepsilon} B_{0} M_{\varepsilon}\right) w\right\|_{H_{\varepsilon}^{1}(Q)} \leqq & \left\|\left(B_{\varepsilon}-B_{0} M_{\varepsilon}\right) w\right\|_{H_{\varepsilon}^{1}(Q)}+\left\|\left(I-I_{\varepsilon}\right) B_{0} M_{\varepsilon} w\right\|_{H_{\varepsilon}^{1}(Q)} \\
\leqq & C_{1}\left[\varepsilon^{\alpha}\left(\|w\|_{H_{\varepsilon}}+\left\|M_{\varepsilon} w\right\|_{H_{\varepsilon}}\right)+\left\|w-M_{\varepsilon} w\right\|_{H_{\varepsilon}}\right] \\
& +C \varepsilon^{\frac{1}{2}}\left\|B_{0} M_{\varepsilon} w\right\|_{H^{2}((0,1)) \times H^{2}((0,1))} \\
\leqq & C\left(\varepsilon^{\alpha}+\varepsilon^{\frac{1}{2}}\right)\|w\|_{H_{\varepsilon}^{1}(Q) .}
\end{aligned}
$$

Likewise, one shows the estimates (9.7) by using (3.3c), (3.6) and the Remarks 3.1 and 3.2 .

To prove the estimate (9.8), we at first need to show that $\left(\lambda I-M_{\varepsilon} I_{\varepsilon} B_{0}\right)$ is an isomorphism of $\mathscr{V}_{0}$. As in Section 8, we write

$$
\lambda I-M_{\varepsilon} I_{\varepsilon} B_{0}=\left(\lambda I-B_{0}\right)\left(I+\left(\lambda I-B_{0}\right)^{-1}\left(B_{0}-M_{\varepsilon} I_{\varepsilon} B_{0}\right)\right) .
$$

Thanks to Remark 3.2, to (9.5) and (9.7), we have

$$
\begin{aligned}
\sup _{\lambda \in \mathscr{U}} \|\left(\lambda I-B_{0}\right)^{-1}\left(B_{0}\right. & \left.-M_{\varepsilon} I_{\varepsilon} B_{0}\right) \|_{\mathscr{L}\left(\mathscr{V}_{0} ; \mathscr{V}_{0}\right)} \\
& \leqq C_{2}\left\|B_{0}-M_{\varepsilon} I_{\varepsilon} B_{0}\right\|_{\mathscr{L}_{\left(\mathscr{V}_{0} ; \mathscr{V}_{0}\right)} \leqq C_{2} C \varepsilon^{\frac{1}{2}} .}
\end{aligned}
$$

Note that the inequality (9.15) still holds with $\mathscr{V}_{0}$ replaced by $H_{0}$. From (9.14) and (9.15), we deduce at once that there exist positive constants $C_{3}$ and $\varepsilon_{1}, 0<\varepsilon_{1} \leqq \varepsilon_{0}$, such that, for $0<\varepsilon \leqq \varepsilon_{1}$,

$$
\sup _{\lambda \in \mathscr{U}}\left\|\left(\lambda I-M_{\varepsilon} I_{\varepsilon} B_{0}\right)^{-1}\right\|_{\mathscr{L}\left(H_{0} ; H_{0}\right)}+\sup _{\lambda \in \bar{U}}\left\|\left(\lambda I-M_{\varepsilon} I_{\varepsilon} B_{0}\right)^{-1}\right\|_{\mathscr{L}\left(\mathscr{\gamma}_{0} ; \mathscr{r}_{0}\right)} \leqq C_{3} .
$$

To prove that $\lambda I-I_{\varepsilon} B_{0} M_{\varepsilon}$ is an isomorphism of $\mathscr{V}_{\varepsilon}$, we argue as in the proof of (8.22). Let $U_{\varepsilon}$ be any element of $\mathscr{V}_{\varepsilon}$. By (9.16), there exists a unique element $v_{1} \in \mathscr{V}_{0}$ such that

$$
M_{\varepsilon} U_{\varepsilon}=\lambda v_{1}-M_{\varepsilon} I_{\varepsilon} B_{0} v_{1}
$$

and

$$
\left\|v_{1}\right\|_{\mathscr{r}_{0}} \leqq C_{3}\left\|M_{\varepsilon} U_{\varepsilon}\right\|_{\mathscr{r}_{0}} \leqq C_{3} C\left\|U_{\varepsilon}\right\|_{H_{\varepsilon}^{1}(Q)}
$$

If we set

$$
w_{\varepsilon}=\lambda^{-1} U_{\varepsilon}+\lambda^{-1} I_{\varepsilon} B_{0} v_{1}
$$

and notice that $M_{\varepsilon} w_{\varepsilon}=\lambda^{-1} M_{\varepsilon} U_{\varepsilon}+\lambda^{-1} M_{\varepsilon} I_{\varepsilon} B_{0} v_{1}=v_{1}$, then $U_{\varepsilon}=\lambda w_{\varepsilon}-I_{\varepsilon} B_{0} M_{\varepsilon} w_{\varepsilon}$. Moreover, by (9.18), (9.19) and the properties of $I_{\varepsilon}$ and $B_{0}$,

$$
\left\|w_{\varepsilon}\right\|_{H_{\varepsilon}^{1}(Q)} \leqq\left|\lambda^{-1}\right|\left\|U_{\varepsilon}\right\|_{H_{\varepsilon}^{1}(Q)}+C\left|\lambda^{-1}\right|\left\|v_{1}\right\|_{H_{\varepsilon}^{1}(Q)}
$$




$$
\leqq\left|\lambda^{-1}\right|\left(1+C_{3} C\right)\left\|U_{\varepsilon}\right\|_{H_{\varepsilon}^{1}(Q)} \leqq C_{4}\left\|U_{\varepsilon}\right\|_{H_{\varepsilon}^{1}(Q)} .
$$

Likewise, since $M_{\varepsilon} \in \mathscr{L}\left(\mathscr{V}_{0} ; H_{0}\right)$, using (9.16), we can still write the decomposition (9.17) when $U_{\varepsilon}$ is replaced by $U_{0} \in \mathscr{V}_{0}$. This implies that, for $U_{0} \in \mathscr{V}_{0}$,

$$
\left(\lambda I-I_{\varepsilon} B_{0} M_{\varepsilon}\right)^{-1} U_{0}=\left(\lambda^{-1}+\lambda^{-1} I_{\varepsilon} B_{0}\left(\lambda I-M_{\varepsilon} I_{\varepsilon} B_{0}\right)^{-1} M_{\varepsilon}\right) \mathscr{V}_{0} .
$$

The proof of $(9.10)$ is very similar to the proof of $(9.16)$.

To prove (9.9), we first observe that

$$
\lambda I-B_{\varepsilon}=\left(\lambda I-I_{\varepsilon} B_{0} M_{\varepsilon}\right)\left(I-\left(\lambda I-I_{\varepsilon} B_{0} M_{\varepsilon}\right)^{-1}\left(B_{\varepsilon}-I_{\varepsilon} B_{0} M_{\varepsilon}\right)\right) \equiv I-\mathscr{B}_{\varepsilon},
$$

where $\mathscr{B}_{\varepsilon} \in \mathscr{L}\left(\mathscr{V}_{\varepsilon} ; \mathscr{V}_{\varepsilon}\right)$. By $(9.6)$ and $(9.8)$, we see that there exists a positive number $\varepsilon_{2}, 0<\varepsilon_{2} \leqq \varepsilon_{1}$, such that, for $0<\varepsilon \leqq \varepsilon_{2},\left\|\mathscr{B}_{\varepsilon}\right\|_{\mathscr{L}\left(\mathcal{V}_{\varepsilon} ; \mathscr{V}_{\varepsilon}\right)} \leqq \frac{1}{2}$, which implies, by $(9.8)$ and (9.20), that (9.9) holds.

To prove $(9.11)$, we note that

$$
\left(\lambda I-B_{\varepsilon}\right)^{-1}-\left(\lambda I-I_{\varepsilon} B_{0} M_{\varepsilon}\right)^{-1}=\left(\lambda I-B_{\varepsilon}\right)^{-1}\left(B_{\varepsilon}-I_{\varepsilon} B_{0} M_{\varepsilon}\right)\left(\lambda I-I_{\varepsilon} B_{0} M_{\varepsilon}\right)^{-1}
$$

and use the estimates (9.6), (9.8) and (9.9).

Likewise, one shows the estimates (9.12) and (9.13).

We remark that the operator $I_{\varepsilon} B_{0} M_{\varepsilon}$ is compact and that the equation

$$
\left(\lambda I-I_{\varepsilon} B_{0} M_{\varepsilon}\right) \bar{u}=0, \quad \bar{u} \in \mathscr{V}_{\varepsilon}\left(\text { respectively } \in \mathscr{V}_{0}\right)
$$

is equivalent to the following:

$$
\begin{array}{ll}
\text { either } & \lambda=0, \\
\text { or } & \hat{\lambda} \neq 0, \quad \bar{u}=\lambda^{-1} I_{\varepsilon} B_{0} \bar{v}, \quad \text { with }\left(\lambda I-M_{\varepsilon} I_{\varepsilon} B_{0}\right) \bar{v}=0, \quad \bar{v} \in \mathscr{V}_{0} .
\end{array}
$$

From (9.21), (9.4) and Lemmas 9.1 and 9.1', we deduce at once that, for any $\eta>0$, for any $v>0$, there exists a positive number $\varepsilon_{0}(v, \eta)$ such that, for $0<\varepsilon \leqq \varepsilon_{0}(v, \eta)$, we have

$$
\begin{array}{r}
\sigma\left(I_{\varepsilon} B_{0} M_{\varepsilon}\right) \cap(\mathbb{C} \backslash B(0, \eta)) \subset \bigcup_{j=1}^{p} B\left(\lambda_{j}, \frac{v}{2}\right) \\
\sigma\left(B_{\varepsilon}\right) \cap(\mathbb{C} \backslash B(0, \eta)) \subset \bigcup_{j=1}^{p} B\left(\lambda_{j}, \frac{v}{2}\right),
\end{array}
$$

where $\lambda_{j}, 1 \leqq j \leqq p(\eta)$ are given in (9.4). We notice that, due to $(9.21)$, the spectrum of $I_{\varepsilon} B_{0} M_{\varepsilon} \in \mathscr{L}\left(\mathscr{V}_{\varepsilon} ; \mathscr{V}_{\varepsilon}\right)$ coincides with the spectrum of $I_{\varepsilon} B_{0} M_{\varepsilon} \in \mathscr{L}\left(\mathscr{V}_{0} ; \mathscr{V}_{0}\right)$ except maybe for the value $\lambda=0$.

If $\sigma_{1}=\sigma_{1}\left(B_{0}\right)$. with $0 \notin \sigma_{1}\left(B_{0}\right)$, is any spectral set of $B_{0}$, then it induces continuous projections $P_{0} \equiv P_{0}\left(\sigma_{1}\right), Q_{0} \equiv Q_{0}\left(\sigma_{1}\right)=I-P_{0}$ on $\mathscr{V}_{0}$ for which the subspaces $P_{0} \mathscr{V}_{0}$ and $Q_{0} \mathscr{V}_{0}$ are invariant under $B_{0}$ and $\sigma\left(B_{0} \mid P_{0} \mathscr{V}_{0}\right)=\sigma_{1}\left(B_{0}\right)$. If $\gamma$ is a closed curve in $\mathbb{C}$ which encloses $\sigma_{1}\left(B_{0}\right)$ and no other points of $\sigma\left(B_{0}\right)$ (in particular, $\gamma$ does not enclose 0 ), then, for any $v \in \mathscr{V}_{0}$, the projection $P_{0}$ is given by

$$
P_{0} v \equiv P_{0}\left(\sigma_{1}\right) v=\frac{1}{2 \pi i} \int_{\gamma}\left(\lambda I-B_{0}\right)^{-1} v d \lambda .
$$


By Lemma 9.1', we can choose $\varepsilon_{\gamma}>0, C_{\gamma}>0$ so that, for $0<\varepsilon \leqq \varepsilon_{\gamma}$, for $\lambda \in \gamma$,

$$
\begin{aligned}
& \sup \left(\left\|\left(\lambda I-M_{\varepsilon} I_{\varepsilon} B_{0}\right)^{-1}\right\|_{\mathscr{L}\left(H_{0} ; H_{0}\right)},\left\|\left(\lambda I-M_{\varepsilon} I_{\varepsilon} B_{0}\right)^{-1}\right\|_{\mathscr{L}\left(\mathscr{V}_{0} ; \mathscr{V}_{0}\right)},\right. \\
& \quad\left\|\left(\lambda I-I_{\varepsilon} B_{0} M_{\varepsilon}\right)^{-1}\right\|_{\mathscr{L}\left(\mathscr{V}_{\varepsilon} ; \mathscr{V}_{\varepsilon}\right)},\left\|\left(\lambda I-I_{\varepsilon} B_{0} M_{\varepsilon}\right)^{-1}\right\|_{\mathscr{L}\left(\mathscr{V}_{0} ; \mathscr{V}_{0}\right)}, \\
& \left\|\left(\lambda I-B_{\varepsilon}\right)^{-1}\right\|_{\mathscr{L}\left(\mathscr{V}_{\varepsilon} ; \mathscr{V}_{\varepsilon}\right)} \leqq C_{\gamma} .
\end{aligned}
$$

Thus, we can define the projection operators $\mathscr{P}_{0}^{\varepsilon}$ on $\mathscr{V}_{\varepsilon}$ or on $\mathscr{V}_{0}$ and $P^{\varepsilon}$ on $\mathscr{V}_{\varepsilon}$ by

$$
\mathscr{P}_{0}^{\varepsilon} \equiv \mathscr{P}_{0}^{\varepsilon}\left(\sigma_{1}, \gamma\right)=\frac{1}{2 \pi i} \int_{\gamma}\left(\lambda I-I_{\varepsilon} B_{0} M_{\varepsilon}\right)^{-1} d \lambda
$$

and

$$
P_{\varepsilon} \equiv P_{\varepsilon}\left(\sigma_{1}, \gamma\right)=\frac{1}{2 \pi i} \int_{\gamma}\left(\lambda I-B_{\varepsilon}\right)^{-1} d \lambda .
$$

We remark that $\mathscr{P}_{0}^{\varepsilon} \mathscr{V}_{\varepsilon}$ and $\mathscr{P}_{0}^{\varepsilon} \mathscr{V}_{0}$ are invariant under $I_{\varepsilon} B_{0} M_{\varepsilon}$ and that $P_{\varepsilon} \mathscr{V}_{\varepsilon}$ is invariant under $B_{\varepsilon}$.

LEMMA 9.2. With the above notation and hypotheses, we have, for $0<\varepsilon \leqq \varepsilon_{\gamma}$,

$$
\mathscr{P}_{0}^{\varepsilon}=\frac{1}{2 \pi i} \int_{\gamma} \lambda^{-1} I_{\varepsilon} B_{0}\left(\lambda I-M_{\varepsilon} I_{\varepsilon} B_{0}\right)^{-1} M_{\varepsilon} d \lambda
$$

and

$$
\mathscr{P}_{0}^{\varepsilon} \mathscr{V}_{\varepsilon}=\mathscr{P}_{0}^{\varepsilon} \mathscr{V}_{0}
$$

Proof. Since $\gamma$ does not enclose 0, the equality (9.27) is a direct consequence of (9.19) and $\left(9.19^{\prime}\right)$, which is well defined since $\left(\lambda I-M_{\varepsilon} I_{\varepsilon} B_{0}\right)$ is an isomorphism of $\mathscr{V}_{0}$ and of $H_{0}$ for $0<\varepsilon \leqq \varepsilon_{\gamma}$. From (9.27) and the fact that $\mathscr{P}_{0}^{\varepsilon}$ is a projection. we deduce that

$$
\mathscr{P}_{0}^{\varepsilon} \mathscr{V}_{\varepsilon}=\left(\mathscr{P}_{0}^{\varepsilon}\right)^{2} \mathscr{V}_{\varepsilon} \subset \mathscr{P}_{0}^{\varepsilon} \mathscr{V}_{0}=\left(\mathscr{P}_{0}^{\varepsilon}\right)^{2} \mathscr{V}_{0} \subset \mathscr{P}_{0}^{\varepsilon} \mathscr{V}_{\varepsilon}
$$

We also need the following auxiliary result:

LEMMA 9.3. Fix $\eta>0$ and suppose that $\lambda_{0} \in \sigma\left(B_{0}\right) \cap(\mathbb{C} \backslash B(0, \eta))$ has multiplicity $d_{0}$. Choose $v>0$ so that $B(0, \eta) \cap B\left(\lambda_{0}, 2 v\right)=\varnothing, \sigma\left(B_{0}\right) \cap B\left(\lambda_{0}, 2 v\right)=\left\{\lambda_{0}\right\}$. Then there exist positive constants $\varepsilon_{1}=\varepsilon_{1}(v, \eta)$ and $c_{1}=c_{1}(v, \eta)$ such that, for $0<\varepsilon \leqq \varepsilon_{1}$, the projection $\mathscr{P}_{0}^{\varepsilon}$ given by (9.25) is well defined, where $\gamma$ is any closed curve in $B\left(\lambda_{0}, v\right) \backslash \dot{B}\left(\lambda_{0}, 3 v / 4\right)$ enclosing $\lambda_{0}$. Moreover, $\operatorname{dim} \mathscr{P}_{0}^{\varepsilon} \mathscr{V}_{0}=d_{0}=\operatorname{dim} \mathscr{P}_{0}^{\varepsilon} \mathscr{V}_{\varepsilon}$ and

$$
\left\|\mathscr{P}_{0}^{\varepsilon}-P_{0}\right\|_{\mathscr{L}\left(\mathscr{V}_{0} ; \mathscr{V}_{0}\right)} \leqq c_{1} \varepsilon^{\frac{1}{2}}
$$

Proof. By Lemma 9.1', there exist positive constants $\varepsilon_{2}=\varepsilon_{2}(v, \eta)$ and $c_{2}=c_{2}(v, \eta)$ such that, for $0<\varepsilon \leqq \varepsilon_{2}$, for any $\lambda \in B\left(\lambda_{0}, v\right) \backslash \stackrel{\circ}{B}\left(\lambda_{0}, 3 v / 4\right)$, we have

$$
\begin{gathered}
\sup \left(\left\|\left(\lambda I-M_{\varepsilon} I_{\varepsilon} B_{0}\right)^{-1}\right\|_{\mathscr{L}\left(\mathscr{V}_{0} ; \mathscr{V}_{0}\right)},\left\|\left(\lambda I-I_{\varepsilon} B_{0} M_{\varepsilon}\right)^{-1}\right\|_{\left.\mathscr{L}_{\left(\mathscr{V}_{0}\right.} ; \mathscr{V}_{0}\right)},\right. \\
\left.\left\|\left(\lambda I-I_{\varepsilon} B_{0} M_{\varepsilon}\right)^{-1}\right\|_{\mathscr{L}\left(\mathscr{V}_{\varepsilon} ; \mathscr{V}_{\varepsilon}\right)},\left\|\left(\lambda I-B_{\varepsilon}\right)^{-1}\right\|_{\mathscr{L}\left(\mathscr{V}_{\varepsilon} ; \mathscr{V}_{\varepsilon}\right)}\right) \leqq c_{2}(v, \eta), \\
\left\|\left(\lambda I-B_{\varepsilon}\right)^{-1}-\left(\lambda I-I_{\varepsilon} B_{0} M_{\varepsilon}\right)^{-1}\right\|_{\mathscr{L}\left(\mathscr{V}_{\varepsilon} ; \mathscr{V}_{\varepsilon}\right) \leqq c_{2}(v, \eta)\left(\varepsilon^{\alpha}+\varepsilon^{\frac{1}{2}}\right),}
\end{gathered}
$$

and

$$
\sup \left(\left\|\left(\lambda I-B_{0}\right)^{-1}-\left(\lambda I-I_{\varepsilon} B_{0} M_{\varepsilon}\right)^{-1}\right\|_{\mathscr{L}\left(\mathscr{\gamma}_{0} ; \mathscr{\gamma}_{0}\right)}\right)
$$




$$
\left.\left\|\left(\lambda I-B_{0}\right)^{-1}-\left(\lambda I-M_{\varepsilon} I_{\varepsilon} B_{0}\right)^{-1}\right\|_{\mathscr{L}\left(\mathscr{V}_{0} ; \mathscr{r}_{0}\right)}\right) \leqq c_{2}(v, \eta) \varepsilon^{\frac{1}{2}}
$$

Now let $\gamma$ be a closed curve in $B\left(\lambda_{0}, v\right) \backslash \grave{B}\left(\lambda_{0}, 3 v / 4\right)$ enclosing $\lambda_{0}$. The projection $P_{0}$ given in (9.23) is well defined. Moreover, by (9.30), the projection $\mathscr{P}_{0}^{\varepsilon}$ given in (9.25) also is well defined. The estimate (9.29) directly follows from (9.32). It now remains to show that $\operatorname{dim} \mathscr{P}_{0}^{\varepsilon} \mathscr{V}_{0}=d_{0}$. We set $Q_{0}=I-P_{0}, Q_{0}^{\varepsilon}=I-\mathscr{P}_{0}^{\varepsilon}$. Following Kato [21], we define $\mathscr{R}_{0}^{\varepsilon}=\left(P_{0}-\mathscr{P}_{0}^{\varepsilon}\right)^{2}$ and observe that there exists a positive constant $\varepsilon_{3}$, $0<\varepsilon_{3} \leqq \varepsilon_{2}$, such that, for $0<\varepsilon \leqq \varepsilon_{3}$, the operator $\left(I-\mathscr{R}_{0}^{\varepsilon}\right)^{-\frac{1}{2}}$ is a well-defined operator which commutes with $P_{0}$ and $\mathscr{P}_{0}^{\varepsilon}$. If we define the operators

$$
\begin{aligned}
U_{0}^{\varepsilon 1} & =P_{0} \mathscr{P}_{0}^{\varepsilon}+Q_{0} Q_{0}^{\varepsilon}, \\
V_{0}^{\varepsilon 1} & =\mathscr{P}_{0}^{\varepsilon} P_{0}+Q_{0}^{\varepsilon} Q_{0}, \\
U_{0}^{\varepsilon} & =U_{0}^{\varepsilon 1}\left(I-\mathscr{R}_{0}^{\varepsilon}\right)^{-\frac{1}{2}}=\left(I-\mathscr{R}_{0}^{\varepsilon}\right)^{-\frac{1}{2}} U_{0}^{\varepsilon 1}, \\
V_{0}^{\varepsilon} & =V_{0}^{\varepsilon 1}\left(I-\mathscr{R}_{0}^{\varepsilon}\right)^{-\frac{1}{2}}=\left(I-\mathscr{R}_{0}^{\varepsilon}\right)^{-\frac{1}{2}} V_{0}^{\varepsilon 1},
\end{aligned}
$$

then $\quad V_{0}^{\varepsilon} U_{0}^{\varepsilon}=U_{0}^{\varepsilon} V_{0}^{\varepsilon}=I, \quad V_{0}^{\varepsilon}=\left(U_{0}^{\varepsilon}\right)^{-1}, \quad U_{0}^{\varepsilon}=\left(V_{0}^{\varepsilon}\right)^{-1} \quad$ and $\quad U_{0}^{\varepsilon} \mathscr{P}_{0}^{\varepsilon}=P_{0} U_{0}^{\varepsilon} \quad$ and $\mathscr{P}_{0}^{\varepsilon} V_{0}^{\varepsilon}=V_{0}^{\varepsilon} P_{0}$. Therefore, the operators $\mathscr{P}_{0}^{\varepsilon}$ and $P_{0}$ are conjugate: $P_{0}=U_{0}^{\varepsilon} \mathscr{P}_{0}^{\varepsilon}\left(U_{0}^{\varepsilon}\right)^{-1}$. As a consequence, the subspaces $\mathscr{P}_{0}^{\varepsilon} \mathscr{V}_{0}$ and $P_{0} \mathscr{V}_{0}$ are isomorphic and have the same dimension $d_{0}$.

REMARK 9.4. In the sequel, we need to compare $U_{0}^{\varepsilon}$ and $V_{0}^{\varepsilon}$ with the identity. In the proof of Lemma 9.3 , we can choose $\varepsilon_{3}>0$ such that $\left\|\left(I-\mathscr{R}_{0}^{\varepsilon}\right)^{-1}\right\|_{\mathscr{L}\left(\boldsymbol{\gamma}_{0} ; \boldsymbol{\gamma}_{0}\right)} \leqq 2$ for $0<\varepsilon \leqq \varepsilon_{3}$ and, by (9.29),

$$
\left\|\left(I-\mathscr{R}_{0}^{\varepsilon}\right)^{-1}-I\right\|_{\mathscr{L}\left(\mathscr{r}_{0} ; \mathscr{r}_{0}\right)}=\left\|\left(I-\mathscr{R}_{0}^{\varepsilon}\right)^{-1} \mathscr{R}_{0}^{\varepsilon}\right\|_{\mathscr{L}\left(\mathscr{r}_{0} ; \mathscr{r}_{0}\right)} \leqq C \varepsilon .
$$

Note that $\left(I-\mathscr{R}_{0}^{\varepsilon}\right)^{-\frac{1}{2}}=I-S_{0}^{\varepsilon}$, where $S_{0}^{\varepsilon}$ is a solution of the equation

$$
S_{0}^{\varepsilon}=\frac{1}{2}\left(\left(I-\left(I-\mathscr{R}_{0}^{\varepsilon}\right)^{-1}\right)+\left(S_{0}^{\varepsilon}\right)^{2}\right) .
$$

Using the contraction mapping theorem, one shows that one can choose $\varepsilon_{3}>0$ small enough to ensure that, for $0<\varepsilon \leqq \varepsilon_{3},(9.35)$ has a unique solution $S_{0}^{\varepsilon}$ in the closed ball $\left\{S \in \mathscr{L}\left(\mathscr{V}_{0} ; \mathscr{V}_{0}\right):\|S\|_{\mathscr{L}\left(\mathscr{V}_{0} ; \mathscr{V}_{0}\right)} \leqq \frac{1}{2}\right\}$. Moreover, thanks to $(9.34)$ and the definition of $S_{0}^{\varepsilon}$, we have $\left\|S_{0}^{\varepsilon}\right\|_{\mathscr{L}\left(\mathscr{r}_{0} ; \mathscr{V}_{0}\right)} \leqq C \varepsilon$, which implies that

$$
\left\|\left(I-\mathscr{R}_{0}^{\varepsilon}\right)^{-\frac{1}{2}}-I\right\|_{\mathscr{L}\left(\mathscr{V}_{0} ; \mathscr{V}_{0}\right)} \leqq C \varepsilon .
$$

Now we can write

$$
\begin{aligned}
\left\|U_{0}^{\varepsilon}-I\right\|_{\mathscr{L}\left(\mathscr{V}_{0} ; \mathscr{V}_{0}\right) \leqq} & \left\|U_{0}^{\varepsilon 1}-I\right\|_{\mathscr{L}\left(\mathscr{V}_{0} ; \mathscr{V}_{0}\right)}\left\|\left(I-\mathscr{R}_{0}^{\varepsilon}\right)^{-\frac{1}{2}}\right\|_{\mathscr{L}\left(\mathscr{V}_{0} ; \mathscr{V}_{0}\right)} \\
& +\left\|\left(I-\mathscr{R}_{0}^{\varepsilon}\right)^{-\frac{1}{2}}-I\right\|_{\left.\mathscr{L}_{\left(\mathscr{V}_{0}\right.} ; \mathscr{V}_{0}\right)},
\end{aligned}
$$

which implies, by (9.29) and (9.36), that

$$
\left\|U_{0}^{\varepsilon}-I\right\|_{\mathscr{L}\left(\mathscr{\Upsilon}_{0} ; \mathscr{V}_{0}\right)} \leqq C \varepsilon^{\frac{1}{2}}
$$

Likewise, one shows that

$$
\left\|V_{0}^{\varepsilon}-I\right\|_{\mathscr{L}\left(\mathscr{V}_{0} ; \mathscr{V}_{0}\right)} \leqq C \varepsilon^{\frac{1}{2}}
$$

Arguing exactly as in the proof of Lemma 9.3, we obtain the following result:

THEOREM 9.5. Assume the hypotheses of Lemma 9.3 are satisfied. Then one can choose 
the constants $\varepsilon_{1}=\varepsilon_{1}(v, \eta)$ and $c_{1}=c_{1}(v, \eta)$ in Lemma 9.3 such that, for $0<\varepsilon \leqq \varepsilon_{1}$, the projection $P_{\varepsilon}$ given by (9.26) is well defined, where $\gamma$ is any closed curve in $B\left(\lambda_{0}, v\right) \backslash \stackrel{\circ}{B}\left(\lambda_{0}, 3 v / 4\right)$ enclosing $\lambda_{0}$. Moreover, $\operatorname{dim} P_{\varepsilon} \mathscr{V}_{\varepsilon}=\operatorname{dim} \mathscr{P}_{0}^{\varepsilon} \mathscr{V}_{\varepsilon}=d_{0}$ and

$$
\left\|P_{\varepsilon}-\mathscr{P}_{0}^{\varepsilon}\right\|_{\mathscr{L}\left(\mathscr{V}_{0} ; \mathscr{V}_{0}\right)} \leqq c_{1}\left(\varepsilon^{\alpha}+\varepsilon^{\frac{1}{2}}\right) .
$$

REMARK 9.6. Exactly as in the proof of Lemma 9.3, we introduce $Q_{\varepsilon}=I-P_{\varepsilon}$ and $\mathscr{R}_{\varepsilon}=\left(\mathscr{P}_{0}^{\varepsilon}-P_{\varepsilon}\right)^{2}$. We observe that we can choose the positive number $\varepsilon_{3}$ in the proof of Lemma 9.3 so that $\left(I-\mathscr{R}_{\varepsilon}\right)^{-\frac{1}{2}}$ is a well-defined operator which commutes with $\mathscr{P}_{0}^{\varepsilon}$ and $P_{\varepsilon}$. Moreover, we have

$$
\begin{gathered}
\left\|\left(I-\mathscr{R}_{\varepsilon}\right)^{-1}\right\|_{\mathscr{L}\left(\mathscr{V}_{\varepsilon} ; \mathscr{V}_{\varepsilon}\right)} \leqq 2, \\
\sup \left(\| I-\mathscr{R}_{\varepsilon}\right)^{-1}-I\left\|_{\mathscr{L}\left(\mathscr{V}_{\varepsilon} ; \mathscr{V}_{\varepsilon}\right)}\right\|\left(I-\mathscr{R}_{\varepsilon}\right)^{-\frac{1}{2}}-I \|_{\mathscr{L}\left(\mathscr{V}_{\varepsilon} ; \gamma_{\varepsilon}\right)} \leqq C\left(\varepsilon+\varepsilon^{2 \alpha}\right) .
\end{gathered}
$$

If we define the operators

$$
\begin{aligned}
U_{\varepsilon}^{1} & =\mathscr{P}_{0}^{\varepsilon} P_{\varepsilon}+Q_{0}^{\varepsilon} Q_{\varepsilon}, \\
V_{\varepsilon}^{1} & =P_{\varepsilon} \mathscr{P}_{0}^{\varepsilon}+Q_{\varepsilon} Q_{0}^{\varepsilon}, \\
U_{\varepsilon} & =U_{\varepsilon}^{1}\left(I-\mathscr{R}_{\varepsilon}\right)^{-\frac{1}{2}}=\left(I-\mathscr{R}_{\varepsilon}\right)^{-\frac{1}{2}} U_{\varepsilon}^{1}, \\
V_{\varepsilon} & =V_{\varepsilon}^{1}\left(I-\mathscr{R}_{\varepsilon}\right)^{-\frac{1}{2}}=\left(I-\mathscr{R}_{\varepsilon}\right)^{-\frac{1}{2}} V_{\varepsilon}^{1},
\end{aligned}
$$

then $V_{\varepsilon} U_{\varepsilon}=U_{\varepsilon} V_{\varepsilon}=I, \quad V_{\varepsilon}=U_{\varepsilon}^{-1}, \quad U_{\varepsilon}=V_{\varepsilon}^{-1} \quad$ and $U_{\varepsilon} P_{\varepsilon}=\mathscr{P}_{0}^{\varepsilon} U_{\varepsilon}, \quad P_{\varepsilon} V_{\varepsilon}=V_{\varepsilon} \mathscr{P}_{0}^{\varepsilon}$, $\mathscr{P}_{0}^{\varepsilon}=U_{\varepsilon} P_{\varepsilon} U_{\varepsilon}^{-1}$. Moreover, we have the following estimates:

$$
\begin{gathered}
\left\|U_{\varepsilon}-I\right\|_{\mathscr{L}\left(\mathscr{V}_{\varepsilon} ; \gamma_{\varepsilon}\right)} \leqq C\left(\varepsilon^{\frac{1}{2}}+\varepsilon^{\alpha}\right), \\
\left\|V_{\varepsilon}-I\right\|_{\mathscr{L}\left(\mathscr{V}_{\varepsilon} ; \mathscr{V}_{\varepsilon}\right)} \leqq C\left(\varepsilon^{\frac{1}{2}}+\varepsilon^{\alpha}\right) .
\end{gathered}
$$

COROLlaRY 9.7. If the hypotheses of Lemma 9.3 are satisfied, then, for $0<\varepsilon \leqq \varepsilon_{1}$, $B_{\varepsilon} \in \mathscr{L}\left(\mathscr{V}_{\varepsilon} ; \mathscr{V}_{\varepsilon}\right)$ has $d_{0}$ eigenvalues $\lambda_{j}^{\varepsilon}, 1 \leqq j \leqq d_{0}$, counted with their multiplicities, in $B\left(\lambda_{0}, v\right)$. If $\mathscr{X}_{0}=\mathscr{P}_{0} \mathscr{V}_{0}$ and if $\mathscr{B}_{\varepsilon} \in \mathscr{L}\left(\mathscr{X}_{0} ; \mathscr{X}_{0}\right)$ is given by $\mathscr{B}_{\varepsilon}=U_{0}^{\varepsilon} U_{\varepsilon} B_{\varepsilon}\left(U_{\varepsilon}\right)^{-1}\left(U_{0}^{\varepsilon}\right)^{-1}$, where $U_{0}^{\varepsilon}$ and $U_{\varepsilon}$ are given in (9.33) and (9.40), then the eigenvalues of $B_{\varepsilon}$ in $B\left(\lambda_{0}, v\right)$ coincide with the eigenvalues of the operator $\mathscr{B}_{\varepsilon}$. Moreover, we have

$$
\left\|\mathscr{B}_{\varepsilon}-B_{0}\right\|_{\mathscr{L}\left(\mathscr{x}_{0} ; \mathscr{X}_{0}\right)} C\left(\varepsilon^{\frac{1}{2}}+\varepsilon^{\alpha}\right) .
$$

In particular, the eigenvalues $\lambda_{j}^{\varepsilon}$ converge to $\lambda_{0}$ as $\varepsilon \rightarrow 0$.

Proof. We set $\mathscr{X}_{0}^{\varepsilon}=\left(U_{0}^{\varepsilon}\right)^{-1}\left(P_{0} \mathscr{V}_{0}\right)=\left(U_{0}^{\varepsilon}\right)^{-1} \mathscr{X}_{0}$. Since $P_{0}$ and $\mathscr{P}_{0}^{\varepsilon}$ are conjugate and $P_{0} \mathscr{X}_{0}=\mathscr{X}_{0}$, we have $\mathscr{P}_{0}^{\varepsilon} \mathscr{X}_{0}^{\varepsilon}=\left(U_{0}^{\varepsilon}\right)^{-1} P_{0} U_{0}^{\varepsilon} \mathscr{X}_{0}^{\varepsilon}=\left(U_{0}^{\varepsilon}\right)^{-1} \mathscr{X}_{0}=\mathscr{X}_{0}^{\varepsilon}$, which implies that $\mathscr{X}_{0}^{\varepsilon} \subset \mathscr{P}_{0}^{\varepsilon} \mathscr{V}_{0}$. Since $\operatorname{dim} \mathscr{X}_{0}^{\varepsilon}=d_{0}=\operatorname{dim} \mathscr{P}_{0}^{\varepsilon} \mathscr{V}_{0}$, we conclude that $\mathscr{X}_{0}^{\varepsilon}=\mathscr{P}_{0}^{\varepsilon} \mathscr{V}_{0}=\mathscr{P}_{0}^{\varepsilon} \mathscr{V}_{\varepsilon}$ and $\mathscr{X}_{0}^{\varepsilon} \subset \mathscr{V}_{0} \cap \mathscr{V}_{\varepsilon}$. Likewise, we set $\mathscr{X}_{\varepsilon}=\left(U_{\varepsilon}\right)^{-1} \mathscr{X}_{0}^{\varepsilon}$. Since $P_{\varepsilon}$ and $\mathscr{P}_{0}^{\varepsilon}$ are conjugate and $\mathscr{P}_{0}^{\varepsilon} \mathscr{X}_{0}^{\varepsilon}=\mathscr{X}_{0}^{\varepsilon}$, we also have $P_{\varepsilon} \mathscr{X}_{\varepsilon}=\mathscr{X}_{\varepsilon}$, which implies that $\mathscr{X}_{\varepsilon}=P_{\varepsilon} \mathscr{V}_{\varepsilon}$.

Note that $B_{\varepsilon}$ (respectively $\left.\left(I_{\varepsilon} B_{0} M_{\varepsilon}\right)\right)$ can be considered as an element of $\mathscr{L}\left(\mathscr{X}_{\varepsilon} ; \mathscr{X}_{\varepsilon}\right)$ (respectively $\mathscr{L}\left(\mathscr{X}_{0}^{\varepsilon} ; \mathscr{X}_{0}^{\varepsilon}\right)$ ). Note also that $U_{\varepsilon}$ (respectively $U_{0}^{\varepsilon}$ ) is an isomorphism from $\mathscr{X}_{\varepsilon}$ onto $\mathscr{X}_{0}^{\varepsilon}$ (respectively $\mathscr{X}_{0}^{\varepsilon}$ onto $\mathscr{X}_{0}$ ). Thus, we can define the mapping $\mathscr{B}_{\varepsilon}=U_{0}^{\varepsilon} U_{\varepsilon} B_{\varepsilon}\left(U_{\varepsilon}\right)^{-1}\left(U_{0}^{\varepsilon}\right)^{-1}$ which belongs to $\mathscr{L}\left(\mathscr{X}_{0} ; \mathscr{X}_{0}\right)$. Since $B_{\varepsilon}$ and $\mathscr{B}_{\varepsilon}$ are conjugate, they have the same eigenvalues. Likewise, we can define the mapping $U_{0}^{\varepsilon}\left(I_{\varepsilon} B_{0} M_{\varepsilon}\right)\left(U_{0}^{\varepsilon}\right)^{-1} \in \mathscr{L}\left(\mathscr{X}_{0} ; \mathscr{X}_{0}\right)$. Moreover, we can write

$$
\begin{aligned}
\left\|\mathscr{B}_{\varepsilon}-B_{0}\right\|_{\mathscr{L}\left(\mathscr{X}_{0} ; \mathscr{X}_{0}\right)} & \\
\qquad & \left\|U_{0}^{\varepsilon}\left(I_{\varepsilon} B_{0} M_{\varepsilon}\right)\left(U_{0}^{\varepsilon}\right)^{-1}-B_{0}\right\|_{\mathscr{L}\left(\mathscr{X}_{0} ; x_{0}\right)} \\
& \quad+\left\|U_{0}^{\varepsilon}\left(U_{\varepsilon} B_{\varepsilon}\left(U_{\varepsilon}\right)^{-1}-\left(I_{\varepsilon} B_{0} M_{\varepsilon}\right)\right)\left(U_{0}^{\varepsilon}\right)^{-1}\right\|_{\mathscr{L}\left(\mathscr{X}_{0} ; x_{0}\right)} .
\end{aligned}
$$


Since, by (9.37), $\left\|U_{0}^{\varepsilon}\right\|_{\mathscr{L}\left(\mathscr{V}_{0 ;} ; \mathscr{V}_{0}\right)} \leqq C$ and $\left\|\left(U_{0}^{\varepsilon}\right)^{-1}\right\|_{\mathscr{L}\left(\mathscr{V}_{0} ; \mathscr{\gamma}_{0}\right)} \leqq C$, for $0<\varepsilon \leqq \varepsilon_{1}$, we also have $\left\|\left(U_{0}^{\varepsilon}\right)^{-1}\right\|_{\mathscr{L}\left(\mathscr{V}_{0} ; \mathscr{F}_{\varepsilon}\right)} \leqq C,\left\|U_{0}^{\varepsilon} \mid \mathscr{V}_{0}\right\|_{\mathscr{L}\left(\boldsymbol{r}_{\varepsilon} ; \boldsymbol{F}_{\varepsilon}\right)} \leqq C$. From these inequalities and (9.43), we derive that

$$
\begin{aligned}
\left\|\mathscr{B}_{\varepsilon}-B_{0}\right\|_{\mathscr{L}\left(\mathscr{T}_{0} ; \mathscr{Y}_{0}\right)} \leqq & C\left\|U_{\varepsilon} B_{\varepsilon}\left(U_{\varepsilon}\right)^{-1}-I_{\varepsilon} B_{0} M_{\varepsilon}\right\|_{\mathscr{L}\left(\mathscr{V}_{\varepsilon} ; \mathscr{V}_{\varepsilon}\right)} \\
& +\left\|U_{0}^{\varepsilon}\left(I_{\varepsilon} B_{0} M_{\varepsilon}\right)\left(U_{0}^{\varepsilon}\right)^{-1}-B_{0}\right\|_{\mathscr{L}_{(}\left(\boldsymbol{V}_{0} ; \mathscr{V}_{0}\right)} .
\end{aligned}
$$

Since $U_{\varepsilon} B_{\varepsilon}\left(U_{\varepsilon}\right)^{-1}-I_{\varepsilon} B_{0} M_{\varepsilon}=\left(U_{\varepsilon}-I\right) B_{\varepsilon}\left(U_{\varepsilon}\right)^{-1}+B_{\varepsilon}\left(\left(U_{\varepsilon}\right)^{-1}-I\right)+B_{\varepsilon}-I_{\varepsilon} B_{0} M_{\varepsilon}$, we obtain, by using the estimates (9.41) and (9.6), that, for $0<\varepsilon \leqq \varepsilon_{1}$,

$$
\left\|U_{\varepsilon} B_{\varepsilon}\left(U_{\varepsilon}\right)^{-1}-I_{\varepsilon} B_{0} M_{\varepsilon}\right\|_{\mathscr{L}\left(\mathcal{V}_{\varepsilon} \gamma_{\varepsilon}\right)} \leqq C\left(\varepsilon^{\alpha}+\varepsilon^{\frac{1}{2}}\right) .
$$

Likewise, by using (9.37) and (9.7), we show that

$$
\left\|U_{0}^{\varepsilon}\left(I_{\varepsilon} B_{0} M_{\varepsilon}\right)\left(U_{0}^{\varepsilon}\right)^{-1}-B_{0}\right\|_{\mathscr{L}\left(\mathscr{V}_{0} ; \mathscr{V}_{0}\right)} \leqq C \varepsilon^{\frac{1}{2}}
$$

Then (9.42) is a direct consequence of (9.44), (9.45) and (9.46). Since $B_{0}$ and $\mathscr{B}_{\varepsilon}$ are two linear mappings from $\mathscr{X}_{0}$ into $\mathscr{X}_{0}$ satisfying (9.42), the $d_{0}$ eigenvalues $\lambda_{j}^{\varepsilon}$ of $\mathscr{B}_{\varepsilon}$ converge to $\lambda_{0}$ as $\varepsilon \rightarrow 0$.

REMARK 9.8. If $\Phi_{0}=\left(\varphi_{01}, \ldots, \varphi_{0 d_{0}}\right)$ is a basis of $\mathscr{X}_{0}$, then $\Phi_{\varepsilon}=\left(\varphi_{\varepsilon 1}, \ldots, \varphi_{\varepsilon d_{0}}\right)$, where $\varphi_{\varepsilon j}=\left(U_{\varepsilon}\right)^{-1}\left(U_{0}^{\varepsilon}\right)^{-1} \varphi_{0 j}$, is a basis of $\mathscr{X}_{\varepsilon}$. Arguing as in the proof of Corollary 9.7, we obtain $\left\|\varphi_{\varepsilon j}-\varphi_{0 j}\right\|_{H_{\varepsilon}^{1}(Q)} \leqq C\left(\varepsilon^{\alpha}+\varepsilon^{\frac{1}{2}}\right)$.

COROLLARY 9.9. Assume that the conditions of Lemma 9.3 are satisfied, but now that $d_{0}=1$; that is, $\lambda_{0}$ is a simple eigenvalue of $B_{0}$. Then, for $0<\varepsilon \leqq \varepsilon_{1}, B_{\varepsilon}$ has one and only one eigenvalue $\lambda_{2}$ in $B\left(\lambda_{0}, v\right)$. This eigenvalue is simple and satisfies the inequality

$$
\left|\lambda_{\varepsilon}-\lambda_{0}\right| \leqq C\left(\varepsilon^{\alpha}+\varepsilon^{\frac{1}{2}}\right) \text {. }
$$

We also can choose an eigenvector $\varphi_{\varepsilon} \in \mathscr{V}_{\varepsilon}$ (respectively $\varphi_{0} \in \mathscr{V}_{0}$ ) corresponding to $\lambda_{\varepsilon}$ (respectively $\lambda_{0}$ ) such that $\left\|\varphi_{\varepsilon}\right\|_{\mathscr{V}_{\varepsilon}}=\left\|\varphi_{0}\right\|_{\mathcal{V}_{0}}=1$ and

$$
\left\|\varphi_{\varepsilon}-\varphi_{0}\right\|_{H_{\varepsilon}^{1}(Q)} \leqq C\left(\varepsilon^{\alpha}+\varepsilon^{\frac{1}{2}}\right) \text {. }
$$

From Lemmas 9.1, 9.1' and Corollary 9.9, we at once deduce the following result:

COROLLARY 9.10. If the operator $B_{0}$ has only simple eigenvalues, then, for any integer $N>0$, there is a positive number $\tilde{\varepsilon}_{1}$ such that, for $0<\varepsilon \leqq \tilde{\varepsilon}_{1}$, the operator $B_{\varepsilon}$ has the first $N$ eigenvalues simple with the ordering being according to nonincreasing modulus.

Proof of Corollary 9.9. The fact that $B_{\varepsilon}$ has only one eigenvalue $\lambda_{\varepsilon}$ in $B\left(\lambda_{0}, v\right)$ and that $\lambda_{\varepsilon}$ is simple and satisfies (9.47) is a direct consequence of Corollary 9.7. Let $\varphi_{0} \in \mathscr{V}_{0}$ be an eigenfunction associated with $\lambda_{0}$ such that $\left\|\varphi_{0}\right\|_{\varkappa_{0}}=1$. If we set

$$
\varphi_{0}^{\varepsilon}=\mathscr{P}_{0}^{\varepsilon} \varphi_{0}, \quad \tilde{\varphi}_{\varepsilon}=P_{\varepsilon} \mathscr{P}_{0}^{\varepsilon} \varphi_{0}, \quad \varphi_{\varepsilon}=\frac{\tilde{\varphi}_{\varepsilon}}{\left\|\tilde{\varphi}_{\varepsilon}\right\|_{\mathscr{Y}_{\varepsilon}}},
$$

then, by Theorem 9.5 and Corollary 9.7, we have

$$
\left\|\varphi_{0}^{\varepsilon}-\varphi_{0}\right\|_{\gamma_{0}}+\left\|\tilde{\varphi}_{\varepsilon}-\varphi_{0}^{\varepsilon}\right\|_{\mathscr{\gamma}_{\varepsilon}} \leqq c\left(\varepsilon^{\frac{1}{2}}+\varepsilon^{\alpha}\right)
$$

On the other hand, arguing as in Sections 5 and 6 and using also (9.2), we can show 
that

$$
\left|\left\|\varphi_{0}\right\|_{\gamma_{0}}-\left\|\varphi_{0}\right\|_{V_{\varepsilon}}\right| \leqq c \varepsilon^{\frac{1}{2}}\left\|\varphi_{0}\right\|_{H^{2}((0,1)) \times H^{2}((0,1))} \leqq \frac{c \varepsilon^{\frac{1}{2}}}{\lambda_{0}} .
$$

Now (9.48) is a straightforward consequence of (9.49), (9.50) and (9.51).

REMARK 9.11. If we suppose that a parameter $\mu$ belongs to a compact set of a metric space and the operators $B_{\varepsilon}=B_{\varepsilon}(\mu)$ depend continuously upon $\mu$ for each $\varepsilon>0$ and the inequalities (9.2), (9.3) hold uniformly in $\mu$, then all of the estimates and results obtained above are valid uniformly in $\mu$.

REMARK 9.12. Let $Y$ be a Banach space and let $\mu_{0} \in Y$. We introduce the operator $B_{0}=B_{0}\left(\mu_{0}\right)$. If $r_{0}$ is a positive number, we denote the ball in $Y$ of centre $\mu_{0}$ and radius $r_{0}$ by $B_{Y}\left(\mu_{0}, r_{0}\right)$. For each $\varepsilon>0,0<\varepsilon \leqq \varepsilon_{0}$, we introduce a subset $M_{\varepsilon}$ of $B_{Y}\left(\mu_{0}, r_{0}\right)$ such that $\mu_{0} \in \bar{M}_{\varepsilon}$ and, for $\mu_{\varepsilon} \in M_{\varepsilon}$, we define an operator $B_{\varepsilon}\left(\mu_{\varepsilon}\right)$. We assume that the operators $B_{0}\left(\mu_{0}\right)$ and $B_{\varepsilon}\left(\mu_{\varepsilon}\right)$ satisfy the hypotheses $(9.1),(9.2)$ and that there exist positive constants $c_{1}, \alpha, \alpha_{1}$, such that, for $0<\varepsilon \leqq \varepsilon_{0}$,

$$
\begin{array}{r}
\left\|B_{\varepsilon}\left(\mu_{\varepsilon}\right)\right\|_{\mathscr{L}\left(\mathscr{H}_{\varepsilon} ; \mathscr{Y}_{\varepsilon}\right)}+\left\|B_{0}\left(\mu_{0}\right)\right\|_{\mathscr{L}\left(L^{2}((0,1)) \times L^{2}((0,1)) ; \mathscr{Y}_{0} \cap\left(H^{2}((0,1)) \times H^{2}((0,1))\right)\right)} \leqq c_{1}, \quad\left(9.2^{\prime}\right) \\
\left\|B_{\varepsilon}\left(\mu_{\varepsilon}\right) \hat{h}_{\varepsilon}-B_{0} \hat{h}_{0}\right\|_{H_{\varepsilon}^{1}(Q)} \leqq c_{1}\left[\left\|\hat{h}_{\varepsilon}-\hat{h}_{0}\right\|_{H_{\varepsilon}}+\left(\left\|\mu_{\varepsilon}-\mu_{0}\right\|_{Y}^{\alpha_{1}}+\varepsilon^{\alpha}\right)\left(\left\|\hat{h}_{\varepsilon}\right\|_{H_{\varepsilon}}+\left\|\hat{h}_{0}\right\|_{H_{\varepsilon}}\right)\right]
\end{array}
$$

for any $\hat{h}_{\varepsilon} \in \mathscr{H}_{\varepsilon}, \hat{h}_{0} \in L^{2}((0,1)) \times L^{2}((0,1))$.

Then, obviously, all of the estimates and results above are still valid if we replace the operators $B_{0}, B_{\varepsilon}$ by $B_{0}\left(\mu_{0}\right), B_{\varepsilon}\left(\mu_{\varepsilon}\right)$, if we replace the term $\left(\varepsilon^{\alpha}+\varepsilon^{\frac{1}{2}}\right)$ by $\left(\varepsilon^{\alpha}+\varepsilon^{\frac{1}{2}}+\left\|\mu_{\varepsilon}-\mu_{0}\right\|_{Y}^{\alpha_{1}}\right)$ in the estimates, if we replace the projection $P_{\varepsilon}$ by $P_{\varepsilon, \mu_{\varepsilon}}$ and if we replace the sentence "there exists a positive number $\varepsilon_{1}, \ldots$ " by the following one: "there exist two positive numbers $\delta_{1}$ and $\varepsilon_{1}$ such that, for $0<\varepsilon \leqq \varepsilon_{1}$, for $\mu_{\varepsilon} \in M_{\varepsilon} \cap B_{Y}\left(\mu_{0}, \delta_{1}\right), \ldots$ "

\section{Applications}

We now apply the above results to our problems $(\tilde{\mathrm{P}})_{0}$ and $(\tilde{\mathrm{P}})_{\varepsilon}$.

Proposition 9.13. For any $v_{0} \in \mathscr{V}_{0}$, the spectrum $\sigma\left(A_{0}+D \hat{f}\left(v_{0}\right)\right)$ of $A_{0}+D \hat{f}\left(v_{0}\right)$ consists only of simple real eigenvalues.

Proof. Since $A_{0}+D \hat{f}\left(v_{0}\right)$ is a selfadjoint operator with compact resolvent, the spectrum $\sigma\left(A_{0}+D \hat{f}\left(v_{0}\right)\right)$ consists only of real eigenvalues. Let us show that they are all simple. We set $v_{0}=\left(v_{01}, v_{02}\right)$. Let $\lambda_{0}$ be an eigenvalue of $A_{0}+D \hat{f}\left(v_{0}\right)$. For $i=1,2$, the space of the solutions of the equation

$$
-\frac{1}{g_{i}}\left(g_{i} v_{i x_{i}}\right)_{x_{i}}+D f\left(v_{0 i}\right) v_{i}=\lambda_{0} v_{i}, \quad v_{i}(1)=0,
$$

is of dimension 1 , given say, by $\mathbb{R} \varphi_{i}$, where $\varphi_{i} \in H^{2}((0,1))$ and $\varphi_{i}^{2}+\varphi_{i x_{i}}^{2} \neq 0$. We note that $\lambda_{0}$ is an eigenvalue of $A_{0}+D \hat{f}\left(v_{0}\right)$ if and only if the system

$$
\mu_{1} \varphi_{1}(0)-\mu_{2} \varphi_{2}(0)=0, \quad \mu_{1} g_{1}(0) \varphi_{1 x_{1}}(0)+\mu_{2} g_{2}(0) \varphi_{2 x_{2}}(0)=0
$$

has a solution $\left(\mu_{1}, \mu_{2}\right) \neq(0,0)$. Since $g_{i}(0) \neq 0$ and $\varphi_{i}^{2}+\varphi_{i x_{i}}^{2} \neq 0$, the space of solutions of $(9.52)$ is at most of dimension 1 . Since $\lambda_{0}$ is an eigenvalue, the dimension is 1 . 
We are going to use the following lemma, the proof of which is very similar to that of [14, Lemma 4.5].

LeMma 9.14. For any positive constant $R_{0}$, there exists a positive constant $k_{0}=k_{0}\left(R_{0}\right)$ such that, for $0<\varepsilon \leqq \varepsilon_{0}$, we have

(i) For any $u_{0} \in B_{H^{1}(Q)}\left(0 ; R_{0}\right), u \in V_{\varepsilon}$,

$$
\left\|D \hat{f}\left(u_{0}\right) u\right\|_{H_{\varepsilon}} \leqq k_{0}\left(R_{0}\right)\|u\|_{V_{\varepsilon}} ;
$$

(ii) for any $v_{0} \in B_{H^{1}((0,1)) \times H^{1}((0,1))}\left(0 ; R_{0}\right), v \in H^{1}((0,1)) \times H^{1}((0,1))$,

$$
\left\|D \hat{f}\left(v_{0}\right) v\right\|_{H_{0}} \leqq k_{0}\left(R_{0}\right)\|v\|_{H^{x}((0,1)) \times H^{1}((0,1))} ;
$$

(iii) for any $u_{0} \in B_{H^{1}(Q)}\left(0 ; R_{0}\right), v_{0} \in B_{H^{1}((0,1)) \times H^{1}((0,1))}\left(0 ; R_{0}\right), u \in H^{1}(Q)$,

$$
\left\|\left(D \hat{f}\left(u_{0}\right)-D \hat{f}\left(v_{0}\right)\right) u\right\|_{H_{s}} \leqq k_{0}\left(R_{0}\right)\left\|u_{0}-v_{0}\right\|_{H^{1}(Q)}\|u\|_{H^{1}(Q)} .
$$

From the estimates $(2.4)_{\varepsilon},(2.4)_{0},(9.53)$ and $(9.54)$, we deduce that there exist positive constants $\tilde{\varepsilon}_{0}, \beta_{0}, \beta$ such that, for $0<\varepsilon \leqq \min \left(\tilde{\varepsilon}_{0}, \varepsilon_{0}\right), u_{0} \in \mathscr{V}_{\varepsilon} \cap B_{H^{1}(Q)}\left(0 ; R_{0}\right)$, $v_{0} \in \mathscr{V}_{0} \cap B_{\left.H^{1}((0,1)) \times H^{1}((0.1))\right)}\left(0 ; R_{0}\right), u \in V_{\varepsilon}, v \in \mathscr{V}_{0}$, we have

$$
\begin{aligned}
a_{\varepsilon}(u, u)+\left(D \hat{f}\left(u_{0}\right) u, u\right)_{H_{\varepsilon}}+\beta_{0}(u, u)_{H_{\varepsilon}} & \geqq \beta\|u\|_{H_{\varepsilon}^{1}(Q)}^{2}, \\
a_{0}(v, v)+\left(D \hat{f}\left(v_{0}\right) v, v\right)_{H_{0}}+\beta_{0}(v, v)_{H_{0}} & \geqq \beta\|v\|_{H^{1}((0,1)) \times H^{1}((0,1))}^{2} .
\end{aligned}
$$

For $u_{0} \in \mathscr{V}_{\varepsilon} \cap B_{H^{1}(Q)}\left(0 ; R_{0}\right), v_{0} \in \mathscr{V}_{0} \cap B_{H^{1}((0,1)) \times H^{1}((0,1))}\left(0 ; R_{0}\right)$, we let

$$
C_{0}\left(v_{0}\right)=A_{0}+D \hat{f}\left(v_{0}\right)+\beta_{0} I, \quad C_{\varepsilon}\left(u_{0}\right)=A_{\varepsilon}+D \hat{f}\left(u_{0}\right)+\beta_{0} I .
$$

Proposition 9.15. There is a positive constant $c_{1}=c_{1}\left(R_{0}\right)$ such that, for $0<\varepsilon \leqq \min \left(\tilde{\varepsilon}_{0}, \varepsilon_{0}\right)$ and any $u_{0} \in \mathscr{V}_{\varepsilon} \cap B_{H^{1}(Q)}\left(0 ; R_{0}\right), v_{0} \in \mathscr{V}_{0} \cap B_{H^{1}((0,1)) \times H^{1}((0,1))}\left(0 ; R_{0}\right)$, $\hat{h}_{\varepsilon} \in \mathscr{H}_{\varepsilon}, \hat{h}_{0} \in L^{2}((0,1)) \times L^{2}((0,1))$, we have:

$$
\begin{gathered}
\left\|C_{\varepsilon}\left(u_{0}\right)^{-1}\right\|_{\mathscr{L}\left(\mathscr{H}_{\varepsilon} ; \mathscr{V}_{\varepsilon}\right)}+\left\|C_{0}\left(v_{0}\right)^{-1}\right\|_{\mathscr{L}\left(\mathscr{H}_{0 ;} ; \mathcal{V}_{0}\right)} \leqq c_{1}, \\
\left\|C_{0}\left(v_{0}\right)^{-1}\right\|_{\mathscr{S}\left(H_{0} ; H^{2}((0,1)) \times H^{2}((0,1))\right)} \leqq c_{1}, \\
\left\|C_{\varepsilon}\left(u_{0}\right)^{-1} \hat{h}_{\varepsilon}-C_{0}\left(v_{0}\right)^{-1} \hat{h}_{0}\right\|_{H_{\varepsilon}^{1}(Q)} \\
\leqq c_{1}\left[\left\|\hat{h}_{\varepsilon}-\hat{h}_{0}\right\|_{H_{\varepsilon}}+\left(\left\|u_{0}-v_{0}\right\|_{H^{1}(Q)}+\varepsilon^{\frac{1}{2}}\right)\left(\left\|\hat{h}_{\varepsilon}\right\|_{H_{\varepsilon}}+\left\|\hat{h}_{0}\right\|_{H_{\varepsilon}}\right)\right] .
\end{gathered}
$$

Proof. From the estimates (9.56), we infer, by using the Lax-Milgram Theorem, that the operators $C_{\varepsilon}\left(u_{0}\right)^{-1}$ and $C_{0}\left(v_{0}\right)^{-1}$ are well defined and satisfy (9.57). The property (9.58) is a direct consequence of (9.57), (9.54) and (2.7).

Setting $u^{\varepsilon}=C_{\varepsilon}\left(u_{0}\right)^{-1} \hat{h}_{\varepsilon}, v=C_{0}\left(v_{0}\right)^{-1} \hat{h}_{0}$ and arguing as in the proof of Lemma 8.1, we can write

$$
\begin{array}{r}
a_{\varepsilon}\left(u^{\varepsilon}-v, u^{\varepsilon}-v\right)+\beta_{0}\left(u^{\varepsilon}-v, u^{\varepsilon}-v\right)_{H_{\varepsilon}}+\left(D \hat{f}\left(u_{0}\right)\left(u^{\varepsilon}-v\right), u^{\varepsilon}-v\right)_{H_{\varepsilon}} \\
=-\left(\left(D \hat{f}\left(u_{0}\right)-D \hat{f}\left(v_{0}\right)\right) v, u^{\varepsilon}-v\right)_{H_{\varepsilon}}+B_{\varepsilon}^{1 *}\left(u^{\varepsilon}, v\right)+B_{\varepsilon}^{2}\left(u^{\varepsilon}, v\right),
\end{array}
$$

where $B_{\varepsilon}^{2}\left(u^{\varepsilon}, v\right)$ is given by $(6.2 c)$ and

$$
\begin{aligned}
B_{\varepsilon}^{1 *}\left(u^{\varepsilon}, v\right)= & \left(\hat{h}_{\varepsilon}-\beta_{0} u^{\varepsilon}-D \hat{f}\left(u_{0}\right) u^{\varepsilon}, v-I_{\varepsilon} v\right)_{H_{2 \varepsilon M}} \\
& +\left(\hat{h}_{0}-\beta_{0} v-D \hat{f}\left(v_{0}\right) v, u^{\varepsilon}-M_{\varepsilon} u^{\varepsilon}\right)_{H_{2 \varepsilon M}} \\
& +\frac{1}{2} \sum_{j=1}^{2} \int_{J_{\varepsilon}^{j}} g_{j}\left(h_{0 j}-\beta_{0} v_{j}-D f\left(v_{0 j}\right) v_{j}\right)\left(v_{j}-u_{j}^{\varepsilon}\right) d x_{1} d x_{2}
\end{aligned}
$$




$$
+\frac{1}{2} \sum_{j=1}^{2} \int_{J_{\varepsilon}^{j}} g_{j}\left(h_{\varepsilon j}-\beta_{0} u_{j}^{\varepsilon}-D f\left(u_{0 j}\right) u_{j}^{\varepsilon}\right)\left(v_{j}(0)-v_{j}\right) d x_{1} d x_{2} .
$$

Arguing as in Section 6 and in the proof of Lemma 8.1, and using Lemma 9.14 as well as the estimates (9.57) and (9.58) and the ellipticity condition (9.56), we derive from $(9.60)$ the estimate $(9.59)$.

Now let $v_{0} \in \mathscr{V}_{0}$. By Proposition 9.13, the spectrum $\sigma\left(A_{0}+D \hat{f}\left(v_{0}\right)\right)$ of $A_{0}+D \hat{f}\left(v_{0}\right)$ consists of a denumerable sequence of simple eigenvalues satisfying

$$
-B_{1}\left(v_{0}\right)<\tilde{\lambda}_{1}\left(v_{0}\right)<\tilde{\lambda}_{2}\left(v_{0}\right)<\ldots<\tilde{\lambda}_{n}\left(v_{0}\right) \rightarrow+\infty,
$$

where $B_{1}\left(v_{0}\right)$ is a positive constant depending only on $v_{0}$.

Let $u_{0} \in \mathscr{V}_{\varepsilon}$. The linear operator $A_{\varepsilon}+D \hat{f}\left(u_{0}\right)$ is selfadjoint with compact resolvent. Therefore its spectrum $\sigma\left(A_{\varepsilon}+D \hat{f}\left(u_{0}\right)\right)$ is composed only of real eigenvalues $\tilde{\lambda}_{j \varepsilon}\left(u_{0}\right)$ which satisfy:

$$
\tilde{\lambda}_{1 \varepsilon}\left(u_{0}\right)<\tilde{\lambda}_{2 \varepsilon}\left(u_{0}\right) \leqq \ldots \leqq \tilde{\lambda}_{n \varepsilon}\left(u_{0}\right) \rightarrow+\infty .
$$

By Proposition 9.15, the operators $B_{0}\left(\mu_{0}\right)=C_{0}\left(v_{0}\right)^{-1}$ and $B_{\varepsilon}\left(\mu_{\varepsilon}\right)=C_{\varepsilon}\left(u_{0}\right)^{-1}$ satisfy the conditions $\left(9.2^{\prime}\right),\left(9.3^{\prime}\right)$ and we can apply the results of the first part of this section and of Remark 9.12. We thus obtain the following theorem:

THEOREM 9.16. For any $v_{0} \in \mathscr{V}_{0}$, for any positive number $n_{0}$ and any positive constant $\delta$, there are positive numbers $\eta\left(v_{0}, n_{0}, \delta\right), \varepsilon\left(v_{0}, n_{0}, \delta\right)$ such that, for $0<\varepsilon \leqq \varepsilon\left(v_{0}, n_{0}, \delta\right)$, for $u_{0} \in B_{H^{1}(Q)}\left(v_{0}, \eta\left(v_{0}, n_{0}, \delta\right)\right) \cap \mathscr{V}_{\varepsilon}$, the first $n_{0}$ eigenvalues $\tilde{\lambda}_{j, \varepsilon}\left(u_{0}\right)$ of $A_{\varepsilon}+D \hat{f}\left(u_{0}\right)$ are simple, contained in $\left[-B_{1}\left(v_{0}\right), \infty\right)$ and satisfy

$$
\max _{1 \leqq j \leqq n_{0}}\left|\tilde{\lambda}_{j}\left(v_{0}\right)-\tilde{\lambda}_{j, \varepsilon}\left(u_{0}\right)\right| \leqq c\left(v_{0}\right)\left(\varepsilon^{\frac{1}{2}}+\eta\left(v_{0}, n_{0}, \delta\right)\right) \leqq \delta,
$$

where $c\left(v_{0}\right)$ is a positive constant depending only on $v_{0}$.

Using the upper semicontinuity of the attractors $\mathscr{A}_{\varepsilon}$, one deduces from Theorem 9.16, as in [14], the following result:

THEOREM 9.17. There exists a positive number $\varepsilon^{*}$ such that, for $0<\varepsilon \leqq \varepsilon^{*}$, if $u_{0}$ is an equilibrium point of $(\tilde{\mathrm{P}})_{\varepsilon}$, then the null space of $A_{\varepsilon}+D \hat{f}\left(u_{0}\right)$ has dimension no more than one.

Arguing as in [14, Section 4], by using Theorem 9.17 and [14, Theorem 2.4], we prove the following property:

THEOREM 9.18. The $\omega$-limit set of any orbit of $(\tilde{\mathrm{P}})_{0}$ is a single equilibrium point. Furthermore, there exists a positive number $\varepsilon^{*}$ such that, for $0<\varepsilon \leqq \varepsilon^{*}$, the $\omega$-limit set of any orbit of $(\tilde{\mathrm{P}})_{\varepsilon}$ is a single equilibrium point.

REMARK 9.19. The same property holds for the damped wave equation on an L-shaped domain (see [17]).

Another application of the first subsection, above, and Theorem 9.16 is the lower semicontinuity of the attractors $\mathscr{A}_{\varepsilon}$. Assume now that all of the equilibrium points of $(\mathrm{P})_{0}$ are hyperbolic. Then, by Corollary $8.3, E_{0}$ and $E_{\varepsilon}$, for $0<\varepsilon \leqq \varepsilon^{*}$, are finite sets of $N_{0}$ elements $v_{l}, 1 \leqq l \leqq N_{0}$, and $u_{l}^{\varepsilon}, 1 \leqq l \leqq N_{0}$, respectively and (8.28) holds. 
For $\varepsilon$ small enough, we can then apply Theorem 9.16 to $v_{0}=v_{1}$ and $u_{0}=u_{1}^{\varepsilon}$. Below, we set $v_{l}=u_{l}^{0}$.

We now introduce the local unstable sets $W_{\text {loc }, 0}^{u}\left(u_{l}^{0}\right)$ and $W_{\text {loc, },}^{u}\left(u_{l}^{\varepsilon}\right)$ of $T_{0}(t)$ and $T_{\varepsilon}(t)$ around the points $u_{l}^{0}$ and $u_{l}^{\varepsilon}$, respectively. For $0 \leqq \varepsilon \leqq \varepsilon_{0}$, if $U_{l}^{\varepsilon}$ is a neighbourhood of $u_{l}^{\varepsilon}$ in $\mathscr{V}_{\varepsilon}$, then

$$
\begin{aligned}
W_{\mathrm{loc}, \varepsilon}^{u}\left(u_{l}^{\varepsilon}: U_{l}^{\varepsilon}\right) & \equiv W_{\mathrm{loc}, \varepsilon}^{u}\left(u_{l}^{\varepsilon}\right) \\
& =\left\{u \in U_{l}^{\varepsilon}: T_{\varepsilon}(-t) u \in U_{l}^{\varepsilon}, t \geqq 0 ; T_{\varepsilon}(-t) u \rightarrow u_{l}^{\varepsilon} \text { in } \mathscr{V}_{\varepsilon} \text { as } t \rightarrow \infty\right\} .
\end{aligned}
$$

Using Theorem 9.16, one proves the following result (see [18] for the details). We do not give the proof here because it is a little long and technical.

Proposirion 9.20. There exist positive constants $\varepsilon_{0}$ and $\rho_{0}$ such that, for $0 \leqq \varepsilon \leqq \varepsilon_{0}$, $1 \leqq l \leqq N_{0}$, there exists a neighbourhood $U_{l}^{\varepsilon}$ of $u_{l}^{\varepsilon}$ in $\mathscr{V}_{\varepsilon}$ such that $W_{\mathrm{loc}, \varepsilon}^{u}\left(u_{l}^{\varepsilon}\right)$ is a $C^{0}$-submanifold of $\mathscr{V}_{\varepsilon}$, the ball $\mathscr{B}_{\mathscr{V}_{e}}\left(u_{l}^{\varepsilon}, \rho_{0}\right)$ is contained in $U_{l}^{\varepsilon}$ and

$$
\max \left(\dot{\delta}_{H_{\varepsilon}^{1}(Q)}\left(W_{\mathrm{loc}, 0}^{u}\left(u_{l}^{0}\right), W_{\mathrm{loc}, \varepsilon}^{u}\left(u_{l}^{\varepsilon}\right)\right), \delta_{H_{\varepsilon}^{1}(Q)}\left(W_{\mathrm{loc}, \varepsilon}^{u}\left(u_{l}^{\varepsilon}\right), W_{\mathrm{loc}, 0}^{u}\left(u_{l}^{0}\right)\right)\right) \leqq c \varepsilon^{\frac{1}{2}} .
$$

One can adapt the proofs of the lower semicontinuity property of [11, Theorems 2.5 and 2.8] and [28, Section 3] to obtain the following result (see also [18]).

THEOREM 9.21. If all of the equilibrium points of $(\mathrm{P})_{0}$ are hyperbolic, then the attractors $\mathscr{A}_{\varepsilon}$ are lower semicontinuous at $\varepsilon=0$; that is $\delta_{H_{\varepsilon}^{1}(Q)}\left(\mathscr{A}_{0}, \mathscr{A}_{\varepsilon}\right) \rightarrow 0$ as $\varepsilon \rightarrow 0$. Moreover, there exist positive constants $\varepsilon_{0}$ and $p$, with $0<p \leqq \frac{1}{2}$, such that, for $0<\varepsilon \leqq \varepsilon_{0}$,

$$
\delta_{H_{\varepsilon}^{1}(Q)}\left(\mathscr{A}_{0}, \mathscr{A}_{\varepsilon}\right)+\delta_{H_{\varepsilon}^{1}(Q)}\left(\mathscr{A}_{\varepsilon}, \mathscr{A}_{0}\right) \leqq c \varepsilon^{p} .
$$

\section{Some generalisations}

\section{More general L-shaped domains}

With essentially no extra effort, it is possible to replace the functions $\varepsilon g_{i}(\cdot)$ in the definition of the L-shaped domain in Section 2 by more general functions $g_{i}(\cdot, \varepsilon)$. In fact, suppose $g_{i} \in C^{3}([0,1] \times[0,1] ;[0, \infty)), i=1,2$, and

$$
\begin{aligned}
& g_{i}\left(\tilde{x}_{i}, 0\right)=0, \quad g_{i 0}\left(\tilde{x}_{i}\right)=\frac{\partial g_{i}}{\partial \varepsilon}\left(\tilde{x}_{i}, 0\right)>0, \\
& g_{i}\left(\tilde{x}_{i}, \varepsilon\right)>0 \quad \text { for } \tilde{x}_{i} \in[0,1], \varepsilon \in(0,1] .
\end{aligned}
$$

We define the general L-shaped domain by

$$
\begin{aligned}
& Q_{\varepsilon}=Q_{\varepsilon}^{1} \cup Q_{\varepsilon}^{2}, \\
& Q_{\varepsilon}^{1}=\left\{\left(\tilde{x}_{1}, \tilde{x}_{2}\right) \in \mathbb{R}^{2}: 0<\tilde{x}_{2}<g_{1}\left(\tilde{x}_{1}, \varepsilon\right), 0<\tilde{x}_{1}<1\right\}, \\
& Q_{\varepsilon}^{2}=\left\{\left(\tilde{x}_{1}, \tilde{x}_{2}\right) \in \mathbb{R}^{2}: 0<\tilde{x}_{1}<g_{2}\left(\tilde{x}_{2}, \varepsilon\right), 0<\tilde{x}_{2}<1\right\} .
\end{aligned}
$$

All of the results of Sections 2-9 remain valid provided that, in the definition of the spaces $\mathscr{V}_{\varepsilon}, \mathscr{H}_{\varepsilon}, H_{\varepsilon}, \ldots$, we replace $g_{i}\left(x_{i}\right)$ by $g_{i}\left(x_{i}, \varepsilon\right) / \varepsilon$ and that, in the definition of the spaces $\mathscr{V}_{0}, H_{0}$, as well as in the definition of the limit problems $(\tilde{\mathrm{P}})_{0}$ and $(\mathrm{P})_{0}$, we replace $g_{i}\left(x_{i}\right)$ by $g_{i 0}\left(x_{i}\right)$. 


\section{Different boundary conditions}

As in [25, Lemma $\left.2.5^{\prime}\right]$, we keep the homogeneous Dirichlet boundary condition on $\Gamma_{\varepsilon}^{1}$ and take the homogeneous Neumann boundary condition on $\Gamma_{\varepsilon}^{2}$. The variational problem $(\tilde{\mathbf{P}})$ is the same but we need to replace the space $H_{\Gamma_{\varepsilon}}^{1}\left(Q_{\varepsilon}\right)$ by $H_{\Gamma_{\varepsilon}^{1}}^{1}\left(Q_{\varepsilon}\right)=\left\{u \in H^{1}\left(Q_{\varepsilon}\right): u=0\right.$ on $\left.\Gamma_{\varepsilon}^{1}\right\}$. We now define the space $V$ by $V=H_{\Gamma^{1}}^{1}\left(Q^{1}\right) \times H^{1}\left(Q^{2}\right)$ and also set

$$
\mathscr{V}_{0}=\left\{\xi=\left(\xi_{1}, \xi_{2}\right) \in H^{1}((0,1)) \times H^{1}((0,1)): \xi_{1}(1)=0, \xi_{1}(0)=\xi_{2}(0)\right\} .
$$

Using the conditions $\xi_{1}(1)=0$, and $\xi_{1}(0)=\xi_{2}(0)$, one shows that $(2.4)_{0}$ is valid for any $v \in \mathscr{V}_{0}$. Arguing as in [25, Lemma 2.5bis], one proves that $(2.4)_{\varepsilon}$ still holds for any $u \in \mathscr{V}_{\varepsilon} \cup \mathscr{V}_{0}$. The problem $(\tilde{\mathrm{P}})_{0}$ does not change with $\mathscr{V}_{0}$ given as above. In the problem $(\mathrm{P})_{0}$, we replace the boundary condition $v_{2}(1, t)=0$ by the boundary condition $v_{2 x_{2}}(1, t)=0$. With these modifications, all of the results of Sections 2-9 hold.

We also may replace the Dirichlet boundary conditions on $\Gamma_{\varepsilon}$ by the homogeneous Neumann boundary conditions. In this case, we replace $-\Delta$ by $-\Delta+\alpha_{0} I$, where $\alpha_{0}>0$ and we replace the dissipative condition (2.2) by

$$
\limsup _{|s| \rightarrow \infty} \frac{-f(s)}{s} \leqq \alpha \leqq \alpha_{0} .
$$

The variational problem $(\tilde{\mathrm{P}})$ is now considered on the space $H^{1}\left(Q_{\varepsilon}\right)$. The corresponding space $V$ is $V=H^{1}\left(Q^{1}\right) \times H^{1}\left(Q^{2}\right)$ and

$$
\mathscr{V}_{0}=\left\{\xi=\left(\xi_{1}, \xi_{2}\right) \in H^{1}((0,1)) \times H^{1}((0,1)): \xi_{1}(0)=\xi_{2}(0)\right\} .
$$

The inequalities $(2.4)_{\varepsilon}$ and $(2.4)_{0}$ obviously hold. In the problem $(\mathrm{P})_{0}$, we replace the boundary conditions $v_{1}(1, t)=v_{2}(1, t)=0$ by the boundary conditions $v_{1 x_{1}}(1, t)=v_{2 x_{2}}(1, t)=0$. Once these changes are made, all of the results of Sections 2-9 hold.

\section{$T$-shaped domains}

By means of an example, we indicate how to generalise the results of Sections 2-9 to thin $T$-shaped domains. The details as well as other generalisations are in [18]. For given functions $g_{i} \in C^{2}([0,1] ;(0, \infty)), i=1,2,3$, we define a $T$-shaped domain as

$$
\begin{aligned}
& Q_{\varepsilon}=Q_{\varepsilon}^{1} \cup Q_{\varepsilon}^{2} \cup Q_{\varepsilon}^{3}, \\
& Q_{\varepsilon}^{1}=\left\{\left(\tilde{x}_{1}, \tilde{x}_{2}\right) \in \mathbb{R}^{2}: 0<\tilde{x}_{2}<\varepsilon g_{1}\left(\tilde{x}_{1}\right), 0<\tilde{x}_{1}<1\right\}, \\
& Q_{\varepsilon}^{2}=\left\{\left(\tilde{x}_{1}, \tilde{x}_{2}\right) \in \mathbb{R}^{2}: 0<\tilde{x}_{1}<\varepsilon g_{2}\left(\tilde{x}_{2}\right), 0<\tilde{x}_{2}<1\right\}, \\
& Q_{\varepsilon}^{3}=\left\{\left(\tilde{x}_{1}, \tilde{x}_{2}\right) \in \mathbb{R}^{2}:-1<\tilde{x}_{1}<\varepsilon g_{2}\left(\tilde{x}_{2}\right), 0<\tilde{x}_{2}<\varepsilon g_{3}\left(\tilde{x}_{1}\right)\right\} .
\end{aligned}
$$

We set $\Gamma_{\varepsilon}=\Gamma_{\varepsilon}^{1} \cup \Gamma_{\varepsilon}^{2} \cup \Gamma_{\varepsilon}^{3}$, where $\Gamma_{\varepsilon}^{3}=\partial Q_{\varepsilon}^{3} \cap\left\{\tilde{x}_{1}=-1\right\}$ and we consider the problem (P) with, for instance, homogeneous Dirichlet boundary conditions on $\Gamma_{\varepsilon}$ and homogeneous Neumann boundary conditions on $\partial Q_{\varepsilon} \backslash \Gamma_{\varepsilon}$.

We transform coordinates to the canonical domain $Q=Q^{1} \times Q^{2} \times Q^{3}$, where $Q^{i}=$ $(0,1) \times(0,1)$. The unknown $u=\left(u_{1}, u_{2}, u_{3}\right)$ consists of three functions satisfying two junction conditions similar to the one which defined $\mathscr{V}_{\varepsilon}$. The inner product $(\cdot, \cdot)_{H_{\varepsilon}}$ is 
defined as follows

$$
(\xi, \zeta)_{H_{\varepsilon}}=\sum_{j=1}^{3}\left[\int_{Q^{i} \backslash J_{\varepsilon}^{i}} g_{i} \xi_{i} \zeta_{i} d x_{1} d x_{2}+\frac{1}{3} \int_{J_{\varepsilon}^{i}} g_{i} \xi_{i} \zeta_{i} d x_{1} d x_{2}\right]
$$

The space $\mathscr{V}_{0}$ is now given by

$$
\begin{aligned}
\mathscr{V}_{0}=\left\{\xi=\left(\xi_{1}, \xi_{2}, \xi_{3}\right)\right. & \in H^{1}((0,1)) \times H^{1}((0,1)) \times H^{1}((0,1)): \\
\xi_{1}(1) & \left.=\xi_{2}(1)=\xi_{3}(1)=0, \xi_{1}(0)=\xi_{2}(0)=\xi_{3}(0)\right\} .
\end{aligned}
$$

The problem $(\mathrm{P})_{0}$ is stated as: Find $v=\left(v_{1}, v_{2}, v_{3}\right) \in \mathscr{V}_{0}$ such that

$$
\begin{gathered}
v_{i t}-\frac{1}{g_{i}}\left(g_{i} v_{i x_{i}}\right)_{x_{i}}=-f\left(v_{i}\right)-G_{i 0} \text { in }(0,1), \quad i=1,2,3, \\
v_{i}(1, t)=0, \quad i=1,2,3, \\
g_{1}(0) v_{1 x_{1}}(0, t)+g_{2}(0) v_{2 x_{2}}(0, t)+g_{3}(0) v_{3 x_{1}}(0, t)=0 .
\end{gathered}
$$

With these modifications, all of the results of Sections 2-9 hold.

\section{From 2-D to 3-D}

For given positive constants $a, b, c$, let $\Omega_{12}=(0, a) \times(0, b), \Omega_{23}=(0, b) \times(0, c)$. For $\varepsilon \in(0,1]$ and given functions $g_{12} \in C^{2}\left(\bar{\Omega}_{12} ;(0, \infty)\right), g_{23} \in C^{2}\left(\bar{\Omega}_{23} ;(0, \infty)\right)$, we define a three-dimensional "L-shaped" domain $Q_{\varepsilon}$ by

$$
\begin{aligned}
Q_{\varepsilon} & =Q_{\varepsilon}^{12} \cup Q_{\varepsilon}^{23}, \\
Q_{\varepsilon}^{12} & =\left\{\left(\tilde{x}_{1}, \tilde{x}_{2}, \tilde{x}_{3}\right) \in \mathbb{R}^{3}: 0<\tilde{x}_{3}<\varepsilon g_{12}\left(\tilde{x}_{1}, \tilde{x}_{2}\right),\left(\tilde{x}_{1}, \tilde{x}_{2}\right) \in \Omega_{12}\right\}, \\
Q_{\varepsilon}^{23} & =\left\{\left(\tilde{x}_{1}, \tilde{x}_{2}, \tilde{x}_{3}\right) \in \mathbb{R}^{3}: 0<\tilde{x}_{1}<\varepsilon g_{23}\left(\tilde{x}_{2}, \tilde{x}_{3}\right),\left(\tilde{x}_{2}, \tilde{x}_{3}\right) \in \Omega_{23}\right\} .
\end{aligned}
$$

The set $J_{\varepsilon}=\overline{Q_{\varepsilon}^{12} \cap Q_{\varepsilon}^{23}}$ is the junction set and is the closure of the open set

$$
J_{\varepsilon}=\left\{\left(\tilde{x}_{1}, \tilde{x}_{2}, \tilde{x}_{3}\right): 0<\tilde{x}_{1}<\varepsilon g_{23}\left(\tilde{x}_{2}, \tilde{x}_{3}\right), 0<\tilde{x}_{2}<b, 0<\tilde{x}_{3}<\varepsilon g_{12}\left(\tilde{x}_{1}, \tilde{x}_{2}\right)\right\} .
$$

We set

$$
\Gamma_{\varepsilon}=\Gamma_{\varepsilon}^{12} \cup \Gamma_{\varepsilon}^{23}, \quad \Gamma_{\varepsilon}^{12}=\partial Q_{\varepsilon}^{12} \cap\left\{\tilde{x}_{1}=a\right\}, \quad \Gamma_{\varepsilon}^{23}=\partial Q_{\varepsilon}^{23} \cap\left\{\tilde{x}_{3}=c\right\} .
$$

We suppose that $f$ still satisfies the conditions $(2.1),(2.2)$, but now we suppose that $0 \leqq \gamma \leqq 1$.

We consider the parabolic problem $(\mathrm{P})$ on $Q_{\varepsilon}$. To discuss the problems $(\mathrm{P})$ or $(\tilde{\mathrm{P}})$, we transform coordinates to the canonical domain

$$
Q \equiv Q^{12} \times Q^{23}=((0, a) \times(0, b) \times(0,1)) \times((0,1) \times(0, b) \times(0, c))
$$

and we define the map $\varphi_{\varepsilon}: \bar{Q}^{12} \cup \bar{Q}^{23} \rightarrow \bar{Q}_{\varepsilon}$ as $\varphi_{\varepsilon} \mid \bar{Q}^{i j}=\varphi_{\varepsilon}^{i j}, j=1,2$, where

$$
\begin{aligned}
& \varphi_{\varepsilon}^{12}:\left(x_{1}, x_{2}, x_{3}\right) \in \bar{Q}^{12} \mapsto\left(x_{1}, x_{2}, \varepsilon g_{12}\left(x_{1}, x_{2}\right) x_{3}\right) \in \bar{Q}_{\varepsilon}^{12}, \\
& \varphi_{\varepsilon}^{23}:\left(x_{1}, x_{2}, x_{3}\right) \in \bar{Q}^{23} \mapsto\left(\varepsilon g_{23}\left(x_{2}, x_{3}\right) x_{1}, x_{2}, x_{3}\right) \in \bar{Q}_{\varepsilon}^{23} .
\end{aligned}
$$

We set $J_{\varepsilon}^{12}=\left(\varphi_{\varepsilon}^{12}\right)^{-1} J_{\varepsilon}, J_{\varepsilon}^{23}=\left(\varphi_{\varepsilon}^{23}\right)^{-1} J_{\varepsilon}$. As in Section 2, (see (2.3)), we can determine functions $x_{1}\left(\varepsilon, x_{2}, x_{3}\right)$ and $x_{3}\left(\varepsilon, x_{1}, x_{2}\right)$ such that

$$
J_{\varepsilon}^{12}=\left\{\left(x_{1}, x_{2}, x_{3}\right) \in Q^{12}: 0<x_{1}<x_{1}\left(\varepsilon, x_{2}, x_{3}\right), 0<x_{2}<b, 0<x_{3}<1\right\},
$$




$$
J_{\varepsilon}^{23}=\left\{\left(x_{1}, x_{2}, x_{3}\right) \in Q^{23}: 0<x_{3}<x_{3}\left(\varepsilon, x_{1}, x_{2}\right), 0<x_{1}<1,0<x_{2}<b\right\} .
$$

We let

$$
x_{1 \varepsilon M}=\max _{0 \leqq x_{2} \leqq b, 0 \leqq x_{3} \leqq 1} x_{1}\left(\varepsilon, x_{2}, x_{3}\right), \quad x_{3 \varepsilon M}=\max _{0 \leqq x_{1} \leqq 1,0 \leqq x_{2} \leqq b} x_{3}\left(\varepsilon, x_{1}, x_{2}\right),
$$

and define $Q_{n \varepsilon M}=Q_{n \varepsilon M}^{12} \times Q_{n \varepsilon M}^{23}$, where

$$
Q_{n \varepsilon M}^{12}=\left(0, n x_{1 \varepsilon M}\right) \times(0, b) \times(0,1), \quad Q_{n \varepsilon M}^{23}=(0,1) \times(0, b) \times\left(0, n x_{3 \varepsilon M}\right),
$$

and $n=1,2$.

As in Section 2, we can define the bilinear forms $a_{\varepsilon}(\cdot, \cdot), a_{0}(\cdot, \cdot)$, the spaces $\mathscr{H}_{\varepsilon}, \mathscr{V}_{\varepsilon}, \mathscr{V}_{0}, \ldots$, with the obvious changes, by taking into account the above remarks. For example,

$$
\begin{aligned}
\mathscr{V}_{0} & =\left\{\xi=\left(\xi_{12}, \xi_{23}\right) \in H^{1}\left(\Omega_{12}\right) \times H^{2}\left(\Omega_{23}\right): \xi_{12}\left(1, x_{2}\right)\right. \\
& \left.=\xi_{23}\left(x_{2}, 1\right)=0, \xi_{12}\left(0, x_{2}\right)=\xi_{23}\left(x_{2}, 0\right)\right\}
\end{aligned}
$$

and the problem $(\mathrm{P})_{0}$ is given by: Find $v=\left(v_{12}, v_{23}\right) \in \mathscr{V}_{0}$ such that

$$
\begin{gathered}
v_{12 t}-\frac{1}{g_{12}} \sum_{i=1}^{2}\left(g_{12} v_{12 x_{i}}\right)_{x_{i}}=-f\left(v_{12}\right)-G_{120} \quad \text { in } \Omega_{12}, \\
v_{23 t}-\frac{1}{g_{23}} \sum_{i=2}^{3}\left(g_{23} v_{23 x_{i}}\right)_{x_{i}}=-f\left(v_{23}\right)-G_{230} \quad \text { in } \Omega_{23}, \\
v_{12}\left(1, x_{2}, t\right)=v_{23}\left(x_{2}, 1, t\right)=0, \quad v_{12}\left(0, x_{2}, t\right)=v_{23}\left(x_{2}, 0, t\right), \\
g_{12}\left(0, x_{2}\right) \frac{\partial v_{12}}{\partial n_{12}}\left(0, x_{2}, t\right)+g_{23}\left(x_{2}, 0\right) \frac{\partial v_{23}}{\partial n_{23}}\left(x_{2}, 0, t\right)=0,
\end{gathered}
$$

where $n_{12}$ (respectively $n_{23}$ ) is the outer normal to $\partial \Omega_{12}$ (respectively $\partial \Omega_{23}$ ). The mapping $M_{\varepsilon}$ (in Section 3 ) is generalised in an obvious way to this case. We can generalise the mapping $I_{\varepsilon}$ in the following manner: for any $v=\left(v_{12}, v_{23}\right) \in \mathscr{V}_{0}$, we define $I_{\varepsilon} v=\left(\tilde{v}_{12}, \tilde{v}_{23}\right)$ by the relation

$$
\begin{aligned}
& \tilde{v}_{12}\left(x_{1}, x_{2}\right) \\
& = \begin{cases}v_{12}\left(x_{1}, x_{2}\right) & x_{1} \in\left(2 x_{1 \varepsilon M}, 1\right], \\
\frac{x_{1}-x_{1 \varepsilon M}}{x_{1 \varepsilon M}} v_{12}\left(x_{1}, x_{2}\right)+\frac{2 x_{1 \varepsilon M}-x_{1}}{x_{1 \varepsilon M}} \frac{1}{x_{1 \varepsilon M}} \int_{0}^{x_{1 \varepsilon M}} v_{12}\left(s, x_{2}\right) d s & x_{1} \in\left(x_{1 \varepsilon M}, 2 x_{1 \varepsilon M}\right], \\
\frac{1}{x_{1 \varepsilon M}} \int_{0}^{x_{1 \varepsilon M}} v_{12}\left(s, x_{2}\right) d s & x_{1} \in\left[0, x_{1 \varepsilon M}\right] ;\end{cases} \\
& \tilde{v}_{23}\left(x_{2}, x_{3}\right) \\
& = \begin{cases}v_{23}\left(x_{2}, x_{3}\right) & x_{3} \in\left(2 x_{3 \varepsilon M}, 1\right], \\
\frac{x_{3}-x_{3 \varepsilon M}}{x_{3 \varepsilon M}} v_{23}\left(x_{2}, x_{3}\right)+\frac{2 x_{3 \varepsilon M}-x_{3}}{x_{3 \varepsilon M}} \frac{1}{x_{1 \varepsilon M}} \int_{0}^{x_{1 \varepsilon M}} v_{12}\left(s, x_{2}\right) d s & x_{3} \in\left(x_{3 \varepsilon M}, 2 x_{3 \varepsilon M}\right], \\
\frac{1}{x_{1 \varepsilon M}} \int_{0}^{x_{1 \varepsilon M}} v_{12}\left(s, x_{2}\right) d s & x_{3} \in\left[0, x_{3 \varepsilon M}\right] .\end{cases}
\end{aligned}
$$


Theorem 2.2 remains valid and the proof follows along the lines described in Sections 3-7. However, the estimate (6.3) is replaced by: for any $p, 2<p<+\infty$,

$$
\|\xi\|_{L^{2}\left(Q_{2 \varepsilon}^{i j}\right)} \leqq C_{p} \varepsilon^{(p-2) / 2 p}\|\xi\|_{H^{1}\left(\Omega_{i j}\right)}, \quad \text { for any } \xi \in H^{1}\left(\Omega_{i j}\right),
$$

where $C_{p}$ is a positive constant depending only on $p$. All of the estimates proved in Sections $4-8$ are still true if we replace $C \varepsilon^{\frac{1}{2}}$ by $C_{p} \varepsilon^{(p-2) / 2 p}$. The results of the first part of Section 9 as well as Lemma 9.14, Propositions 9.15, 9.20 and Theorem 9.21 still hold once we replace $C \varepsilon^{\frac{1}{2}}$ by $C_{p} \varepsilon^{(p-2) / 2 p}, 2<p<\infty$. Note that the analogues of Proposition 9.13, Theorems 9.16, 9.17, 9.18 are no longer true.

\section{References}

1 A. V. Babin and M. I. Vishik. Regular attractors of semigroups of evolutionary equations. $J$. Math. Pures Appl. 62 (1983), 441-491.

2 F. Bourquin and P. G. Ciarlet. Modeling and justification of eigenvalue problems for junctions between elastic structures. J. Funct. Anal. 87 (1989), 392-427.

3 J. Céa and G. Geymonat. Une méthode de linéarisation via l'optimisation. Symposia Matematica 10 (1972), 431-451.

4 P. G. Ciarlet. Plates and Junctions in Elastic Multi-structures: an Asymptotic Analysis, Collection RMA14 (Paris: Masson-Springer, 1990).

5 P. G. Ciarlet, H. Le Dret and R. Nzengwa. Modélisation de la jonction entre un corps élastique tridimensionnel et une plaque. C.R. Acad. Sci. Paris, Sér. 1305 (1987), 55-58.

6 P. G. Ciarlet, H. Le Dret and R. Nzengwa. Junctions between three-dimensional and two-dimensional linearly elastic structures. J. Math. Pures Appl. 68 (1989), 261-295.

7 D. Cioranescu and J. Saint Jean Paulin. Reinforced and honey-comb structures. J. Math. Pures Appl. 65 (1986), 403-422.

8 M. Crouzeix and J. Rappaz. On Numerical Approximation in Bifurcation Theory, Collection RMA 13 (Paris: Masson, 1990).

9 J. K. Hale. Asymptotic behavior and dynamics in infinite dimensions. In Research Notes in Mathematics 132, 1-41 (Boston: Pitman, 1985).

10 J. K. Hale. Asymptotic Behavior of Dissipative Systems, Mathematical Surveys and Monographs 25 (Providence, R.I.: American Mathematical Society, 1988).

11 J. K. Hale and G. Raugel. Lower semicontinuity of attractors of gradient systems and applications. Ann. Mat. Pura Appl. (IV) 154 (1989), 281-326.

12 J. K. Hale and G. Raugel. Partial differential equations on thin domains, Proc. Int. Conf. in Alabama, 1990. In Difjerential Equations and Mathematical Physics, ed. C. Bennewitz, 63-97 (New York: Academic Press, 1991).

13 J. K. Hale and G. Raugel. Reaction-diffusion equation on thin domains. J. Math. Pures Appl. 71 (1992), 33-95.

14 J. K. Hale and G. Raugel. Convergence in gradient like systems and applications. Z. Angew. Math. Phys. 43 (1992), 63-124.

15 J. K. Hale and G. Raugel. A damped hyperbolic equation on thin domains. Trans. Amer. Math. Soc. 329 (1992), 185-219.

16 J. K. Hale and G. Raugel. Dynamics on thin domains (Preprint).

17 J. K. Hale and G. Raugel. Attractors and convergence of PDE on thin L-shaped domains. In Progress in Partial Differential Equations: the Metz Surveys 2, ed. M. Chipot, 149-171 (Harlow: Longman, 1993).

18 J. K. Hale and G. Raugel. Some additional remarks on PDE on thin L-shaped domains (in preparation).

19 D. Henry. Geometric Theory of Semilinear Parabolic Equations, Lecture Notes in Mathematics 840 (Berlin: Springer, 1981).

20 G. A. Iosif'yan, O. A. Oleinik and A. S. Shamaev. On the limiting behavior of the spectrum of a sequence of operators defined on different Hilbert spaces, Russian Math. Surv. 44 (1989), 195-196.

21 T. Kato. Perturbation Theory for Linear Operators (Berlin and New York: Springer, 1966).

22 J. E. Lagnese and J. L. Lions. Modelling, Analysis and Control of Thin Plates, Collection RMA 6 (Paris: Masson, 1988).

23 H. Le Dret. Modeling of the junction between two rods. J. Math. Pures Appl. 68 (1989), 365-397.

24 H. Le Dret. Folded plates revisited. Comput. Mech. 5 (1989), 345-365. 
25 H. Le Dret. Problèmes Variationnels dans les Multi-domaines-Modélisation des Jonctions et Applications, Collection RMA 19 (Paris: Masson, 1991).

26 J. L. Lions. Perturbations singulières dans les problèmes aux limites et en contrôle optimal, Lecture Notes in Mathematics 323 (Berlin: Springer, 1973).

27 A. G. Ramm. Limit of the spectra of the interior Neumann problems when a solid domain shrinks to a plane one. J. Math. Anal. Appl. 108 (1985), 107-112.

28 A. Raoult. Asymptotic modeling of the elastodynamics of a multi-structure. Asymptotic Anal. 6 (1992), 73-108.

29 G. Raugel. Persistence of Morse-Smale properties under some approximations and singular perturbations (Preprint).

30 G. Raugel and G. Sell. Navier-Stokes equations on thin 3D domains I. Global attractors and regularity of solutions. J. Amer. Math. Soc. 6 (1993), 503-568.

31 G. Raugel and G. Sell. Navier-Stokes equations on thin 3D domains II: Global regularity of spatially periodic solutions, Séminaire $d u$ Collège de France, ed. J. L. Lions (Boston: Longman, to appear).

(Issued 14 April 1994) 